\title{
A biodiversity hotspot for Microgastrinae (Hymenoptera, Braconidae) in North America: annotated species checklist for Ottawa, Canada
}

\author{
Jose Fernandez-Triana', Caroline Boudreault', Joel Buffam', Ronald Mclean' \\ I Canadian National Collection of Insects, Ottawa, Canada \\ Corresponding author: Jose Fernandez-Triana (jose.fernandez@agr.gc.ca)
}

Academiceditor:C.vanAchterberg|Received13September2016|Accepted3November2016|Published17November2016

http://zoobank.org/DEFC1530-7241-4BA6-B778-CA63DB45B422

Citation: Fernandez-Triana J, Boudreault C, Buffam J, Mclean R (2016) A biodiversity hotspot for Microgastrinae (Hymenoptera, Braconidae) in North America: annotated species checklist for Ottawa, Canada. ZooKeys 633: 1-93. doi: $10.3897 /$ zookeys.633.10480

\begin{abstract}
Microgastrinae wasps (Hymenoptera, Braconidae) from the city of Ottawa and its surroundings (a 50$\mathrm{km}$ radius circle, $-7,800 \mathrm{~km}^{2}$ ) were studied based on 1,928 specimens collected between 1894 and 2010, and housed in the Canadian National Collection of Insects. A total of 158 species from 21 genera were identified, which is by far the highest number of species ever recorded for a locality in North America. An annotated checklist of species is provided. Choeras parasitellae (Bouché, 1834) and Pholetesor nanus (Reinhard, 1880) are recorded for the first time in the Nearctic (previously only known from the Palearctic region), Cotesia depressa (Viereck, 1912) is recorded for the first time in Canada (previously only known from the United States), and Cotesia hemileucae (Riley, 1881) and Protapanteles phlyctaeniae (Muesebeck, 1929) are recorded for the first time in the province of Ontario. In Ottawa the most diverse genera are Cotesia, Apanteles, Microplitis, Pholetesor, Microgaster, and Dolichogenidea, altogether comprising $77 \%$ of the species found in the area. A total of 73 species (46\%) were represented by only one or two specimens, suggesting that the inventory for Ottawa is still relatively incomplete. Seasonal distribution showed several peaks of activity, in spring, summer, and early fall. That general pattern varied for individual species, with some showing a single peak of abundance either in the summer or towards the end of the season, others species attaining two peaks, in late spring and late summer, or in early summer and early fall, and yet others attaining up to three different peaks, in spring, summer and fall. At least 72 of the Microgastrinae species from Ottawa have been previously associated with 554 species of Lepidoptera as hosts - but those historical literature records are not always reliable and in many cases are based on data from areas beyond Ottawa. Thus, our knowledge of the associations between the 158 species of microgastrine parasitoids and the caterpillars of the 2,064 species of Lepidoptera recorded from Ottawa is still very incomplete.
\end{abstract}

Copyright Jose Fernandez-Triana et al. This is an open access article distributed under the terms of the Creative Commons Attribution License (CC BY 4.0), which permits unrestricted use, distribution, and reproduction in any medium, provided the original author and source are credited. 


\section{Keywords}

Microgastrinae, diversity, North America, Canada, Ottawa

\section{Introduction}

Microgastrinae wasps are the second largest subfamily of Braconidae (Hymenoptera) (Yu et al. 2016), and evidence suggests they may eventually prove to be the largest subfamily (e.g., Rodriguez et al. 2013). The number of described species has increased significantly within the past 35 years: in 1980 there were about 1,500 (Papp 1976, Mason 1981), by 2012 there were more than 2,200 (Yu et al. 2012), and currently the total surpasses 2,700 (Yu et al. 2016). The actual diversity worldwide is estimated to be 20,000-46,000 species (Rodriguez et al. 2013), meaning that most likely $10 \%$ or even less of the extant species have been described.

At the regional or local level our knowledge about Microgastrinae is also very incomplete, with few biodiversity inventories available and most published information not being comprehensive enough to capture the actual diversity of species. Examples in North America include Lewis and Whitfield (1999), Whitfield and Lewis (2001), Shaw (2002), and Fernandez-Triana et al. (2009, 2011).

Besides its extraordinary diversity, microgastrine wasps are very important in biocontrol efforts against Lepidoptera pests, as they represent the single most important group of caterpillar parasitoids in the world (Whitfield 1997).

This paper analyzes the diversity of Microgastrinae of Ottawa (Canada) and the surrounding areas, discusses the significance of these results for future studies of the group in the Nearctic region, and provides an initial reference point for the Ottawa fauna (with a dynamic species checklist to be available online as well).

\section{Methods}

Since 1895, the Ottawa Field-Naturalists' Club (http://www.ofnc.ca/) has considered a circle with a $50 \mathrm{~km}$ radius (approximately 7,800 $\mathrm{km}^{2}$ ) centered on the Peace Tower in downtown Ottawa as its study area. This human-defined region (variously named as 'National Capital Region', 'Ottawa-Gatineau Region', 'Ottawa Region', 'Ottawa District') has been widely adopted and used in many scientific papers and conservation efforts conducted in the city over the past 120 years. We follow the same concept, henceforth calling it simply 'Ottawa' for brevity. Figure 1 shows the localities within this $50-\mathrm{km}$ radius where specimens of Microgastrinae had been collected and were available to us for study. For consistency, we do not include data from a few additional species collected in localities slightly out of this $100 \mathrm{~km}$ diameter circle even though, from a natural perspective, those species are very likely to be found in Ottawa as well.

This paper is based on the study of 1,928 specimens, housed in the Canadian National Collection of Insects, Ottawa (CNC); they were all databased and assigned to 


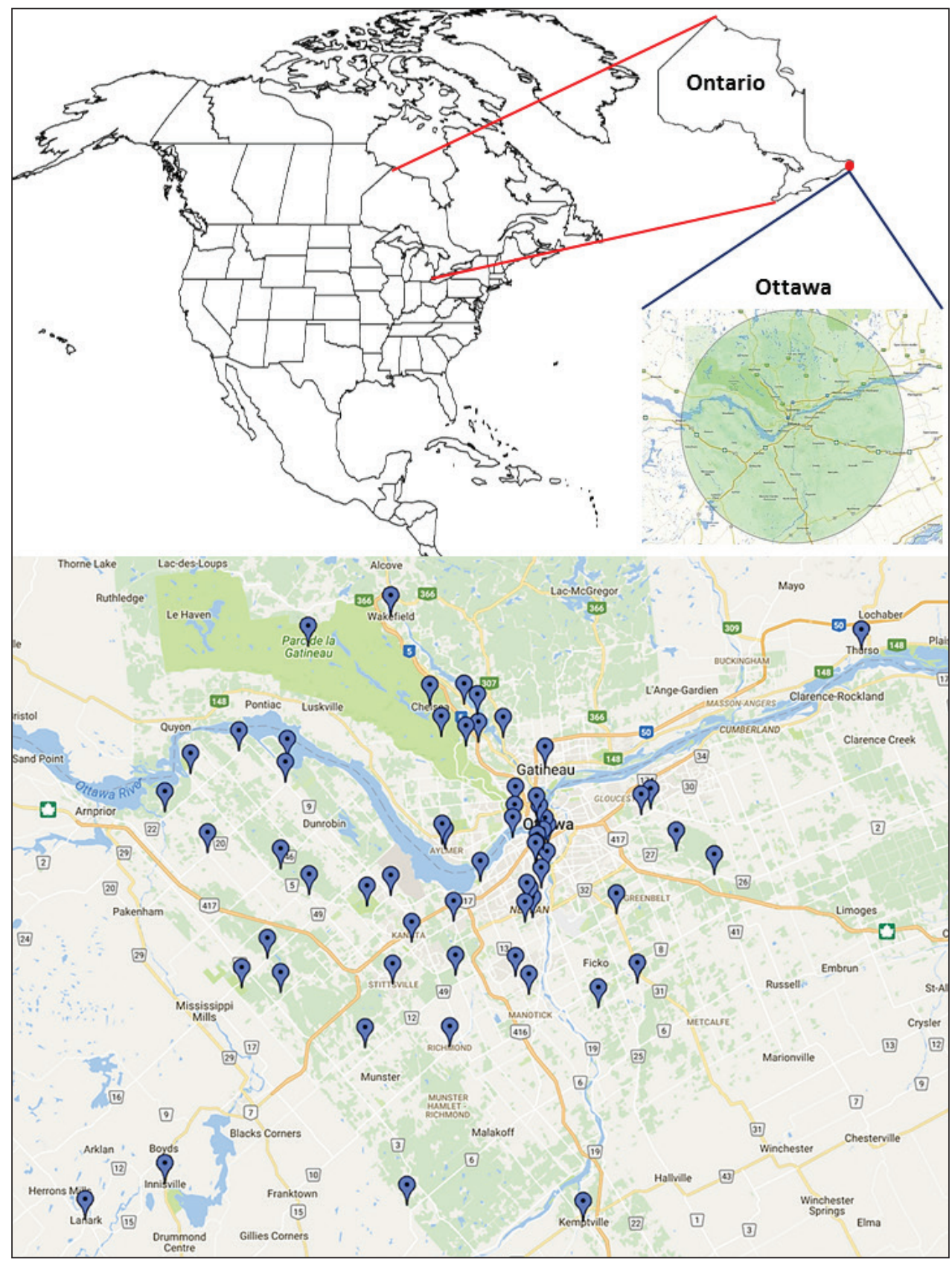

Figure I. Localities within the city of Ottawa and surroundings where specimens of Microgastrinae were collected (1894-2010, data based on CNC holdings only).

species following the most recent taxonomic information available for Canada (Fernandez-Triana 2010, 2014, 2015, Fernandez-Triana et al. 2013, 2014a, 2014 b, 2015). Some specimens could only be identified to genus but in all cases they had unique 
morphological characteristics and/or DNA barcodes that clearly identified them as distinct species-in those cases we use an alphanumeric species identifier e.g., 'Apanteles jft09'. In order to allow these provisional species to be recognized and studied further in the future we also provide DNA Barcodes Index Numbers (BINs) (Ratnasingham and Hebert 2013) for them in the annotated species checklist.

Pictures of 36 species are provided to illustrate the diversity of microgastrine wasps in Ottawa. Photos were taken either with a Leica M165 C or a Keyence VHX-1000 Digital Microscope, using a lens with a range of 10-130 ×. Multiple images were taken of a structure through the focal plane and then combined to produce a single in-focus image. For the images taken with the Leica camera, the Zerene Stacker program (http:// zerenesystems.com/cms/stacker) was used; software associated with the Keyence System produced focused images taken with that camera. Images were corrected using Adobe Photoshop CS4 and the plates were prepared using Microsoft PowerPoint 2010.

A species checklist was generated using the CNC database (http://www.cnc-ottawa.ca/taxonomy/TaxonMain.php). The list is organized alphabetically by genus and species within a given genus. For every taxon we detail general distribution (outside Ottawa), specimens examined, and notes on species where relevant. For zoogeographic regions we used the following acronyms: NEA-Nearctic, NEO-Neotropics, OTL-Oriental, and PAL-Palearctic.

Using the Scratchpad platform and infrastructure (Smith et al. 2011), the species checklist given here as the initial reference point for the Ottawa fauna of Microgastrinae will be made available online (http://microgastrinae.myspecies.info/content/microgastrinae-ottawa-canada), where updates will be maintained as and when changes occur.

\section{Results}

A total of 158 species from 21 genera of Microgastrinae are recorded from Ottawa. Choeras parasitellae (Bouché, 1834) and Pholetesor nanus (Reinhard, 1880) are new records for the Nearctic (previously only known from the Palearctic region). Cotesia depressa (Viereck, 1912) is a new record for Canada (previously only known from the United States). Cotesia hemileucae (Riley, 1881) and Protapanteles phlyctaeniae (Muesebeck, 1929) are new records for Ontario.

The most diverse genera were Cotesia (32 species), Apanteles (31), Microplitis (23), Pholetesor (13), Microgaster (11), and Dolichogenidea (11). These six genera together comprised $77 \%$ of the species found in the area. This pattern is similar to that found in both the whole Nearctic region (Whitfield 1995, Yu et al. 2012, 2016) as well as in specific North American localities that have been the focus of biodiversity inventories (Lewis and Whitfield 1999, Whitfield and Lewis 2001, Shaw 2002, Fernandez-Triana et al. 2009, 2011). A notable exception is Glyptapanteles, one of the most diverse genera in North America and elsewhere, but currently with only three species recorded from Ottawa. However, many species of that genus still remain unidentified and further study will certainly reveal a much higher diversity for Glyptapanteles in the area. Five 
genera of Microgastrinae reached their northernmost known distribution in Ottawa: Alphomelon, Clarkinella, Distatrix, Protomicroplitis and Pseudapanteles (FernandezTriana 2010, 2014, 2015, Fernandez-Triana et al. 2014c).

At species level, the most common were Glyptapanteles militaris (Walsh, 1861) (322 specimens), Pholetesor ornigis (Weed, 1887) (157), Glyptapanteles pallipes (Reinhard, 1880) (87), Apanteles nephoptericis (Packard, 1864) (82), Cotesia atalantae (Packard, 1881) (77), Microplitis varicolor Viereck, 1917 (72), Hypomicrogaster zonaria (Say, 1836) (65), Dolichogenidea cacoeciae (Riley, 1881) (59), Hygroplitis melligaster (Provancher, 1886) (47), and Cotesia laeviceps (Ashmead, 1890) (45). Those ten species together represented 53\% of all Microgastrinae specimens collected in Ottawa.

There were 50 species $(31.4 \%)$ represented by only one specimen, and $23(14.4 \%)$ represented by two specimens. The high proportion (45.9\%) of singletons and doubletons shows how incomplete the Ottawa species inventory still is. The Chao-1 nonparametric estimator of species richness (Rarefaction Calculator at http://www.biology.ualberta.ca/jbrzusto/rarefact.php\#Calculator) estimates $213.35 \pm 16.29$ species for Ottawa, thus our present knowledge represents less than $75 \%$ of the actual total of species to be found there.

Among all listed species, 73 were only identified to genus and have been assigned interim alphanumeric identifiers. Some of them certainly represent new taxa to be described in future papers. Others may represent species already described in Europe and for the present are given interim identifiers until they are compared with Palearctic material.

Regarding zoogeographical affinities, 132 species (83\%) found in Ottawa are strictly Nearctic, 16 species (10\%) are distributed within the Holarctic, $5(3 \%)$ are recorded from the New World (Nearctic and Neotropics), and $10(6 \%)$ are either truly cosmopolitan or present in at least three different regions (Nearctic/Neotropics/ Palearctic or Nearctic/Oriental/Palearctic).

Some of the localities sampled contain special, sometimes unique habitats/ecosystems. Gatineau Park, with a rich biodiversity and a wide range of protected habitats and ecosystems within its 36,000 ha (http://www.ncc-ccn.gc.ca/places-to-visit/ gatineau-park/conservation-gatineau-park) contains five species so far found nowhere else within the Ottawa area: Alphomelon winniewertzae Deans, 2003, Cotesia depressa (Viereck, 1912), Cotesia diacrisiae (Gahan, 1917), Distatrix carolinae Fernandez-Triana, 2010, and Microplitis impressus (Wesmael, 1837).

Mer Bleue, a 3,500 ha conservation area with a northern, boreal-like ecosystem more typical of the Subarctic than the Ottawa Valley (http://www.ncc-ccn.gc.ca/ places-to-visit/greenbelt/mer-bleue) harbours three species that have not been found elsewhere in the Ottawa area: Cotesia clisiocampae (Ashmead 1903), Dolichogenidea absona (Muesebeck, 1965), and Pholetesor rhygoplitoides Whitfield, 2006. Similarly, the approximately 1,500 ha of alvar ecosystem near Almonte (Belcher et al. 1992) is the only known Ottawa-area locality for Pholetesor viminetorum (Wesmael, 1837). Further studies on the fauna of Microgastrinae may show that some of those species are more widely distributed within Ottawa or beyond, though some are likely to remain restricted to particular habitats or ecosystems where their hosts occur. 
Table I. Microgastrinae diversity by country with over 100 species recorded. Data from Taxapad (Yu et al. 2012), Fauna Europaea (http://www.fauna-eu.org/) and the present paper (for Canada and Ottawa). For Costa Rica the total is a very conservative estimate based on numerous papers published after 2012 on the fauna of Area de Conservación Guanacaste (http://janzen.sas.upenn.edu/caterpillars/database.lasso).

\begin{tabular}{l|l|l|l}
\hline Costa Rica & $400+$ & Finland & 163 \\
\hline Hungary & 328 & Ottawa & 158 \\
\hline China & 316 & Mongolia & 157 \\
\hline Russia & 293 & Italy & 150 \\
\hline U.S.A. & 284 & Ukraine & 149 \\
\hline Germany & 249 & Yugoslavia & 139 \\
\hline United Kingdom & 234 & Democratic Republic of Congo & 134 \\
\hline India & 210 & Bulgaria & 124 \\
\hline Canada & 196 & Korea & 124 \\
\hline Czech Republic & 190 & France & 123 \\
\hline Romania & 173 & Sweden & 117 \\
\hline Poland & 170 & Moldova & 113 \\
\hline Switzerland & 168 & Slovakia & 113 \\
\hline Turkey & 167 & Australia & 112 \\
\hline Netherlands & 176 & Kazakhstan & 112 \\
\hline & & &
\end{tabular}

Table 2. Microgastrinae diversity in selected North American localities. Data from Lewis and Whitfield (1999), Whitfield and Lewis (2001), Shaw (2002), Fernandez-Triana et al. (2009, 2011), and the present paper.

\begin{tabular}{l|c|c}
\hline Locality/ecosystem & Latitude & Species recorded \\
\hline Yellowstone National Park, Montana/forests & $44^{\circ} \mathrm{N}$ & 35 \\
\hline Quebec/apple orchards & $45^{\circ} \mathrm{N}$ & 36 \\
\hline Midwestern USA/tall grass prairies & $39^{\circ} \mathrm{N}$ & 55 \\
\hline Arkansas/forests & $34^{\circ} \mathrm{N}$ & 65 \\
\hline Churchill, Manitoba/boreal forest/tundra & $59^{\circ} \mathrm{N}$ & 79 \\
\hline Ottawa, Ontario/diverse habitats & $45^{\circ} \mathrm{N}$ & 158 \\
\hline
\end{tabular}

The diversity of Microgastrinae revealed for Ottawa is extraordinary: in spite of being a relatively small area $\left(7,800 \mathrm{~km}^{2}\right)$ its species total would rank $17^{\text {th }}$ among countries of the world (Table 1). And, when placed within the context of what has been published for other, similarly-sized localities in North America, Ottawa has the highest number of species, double the second most diverse locality (Table 2). Even when accounting for differences in collecting efforts and taxonomic coverage (e.g., it is clear that the relatively low diversity in the Yellowstone National Park is due to insufficient study of its Microgastrinae fauna), the total recorded in Ottawa may not be surpassed by any North American locality at a comparable latitude. The high diversity found in Ottawa likely relates to being a transition from an eastern deciduous forest biome to a boreal biome, with small areas of unusual habitats like dunes, alvars, floodplains, and bogs.

We are not aware of published information for more southern localities, although based on specimens seen in collections (Fernandez-Triana, unpublished), it is clear 


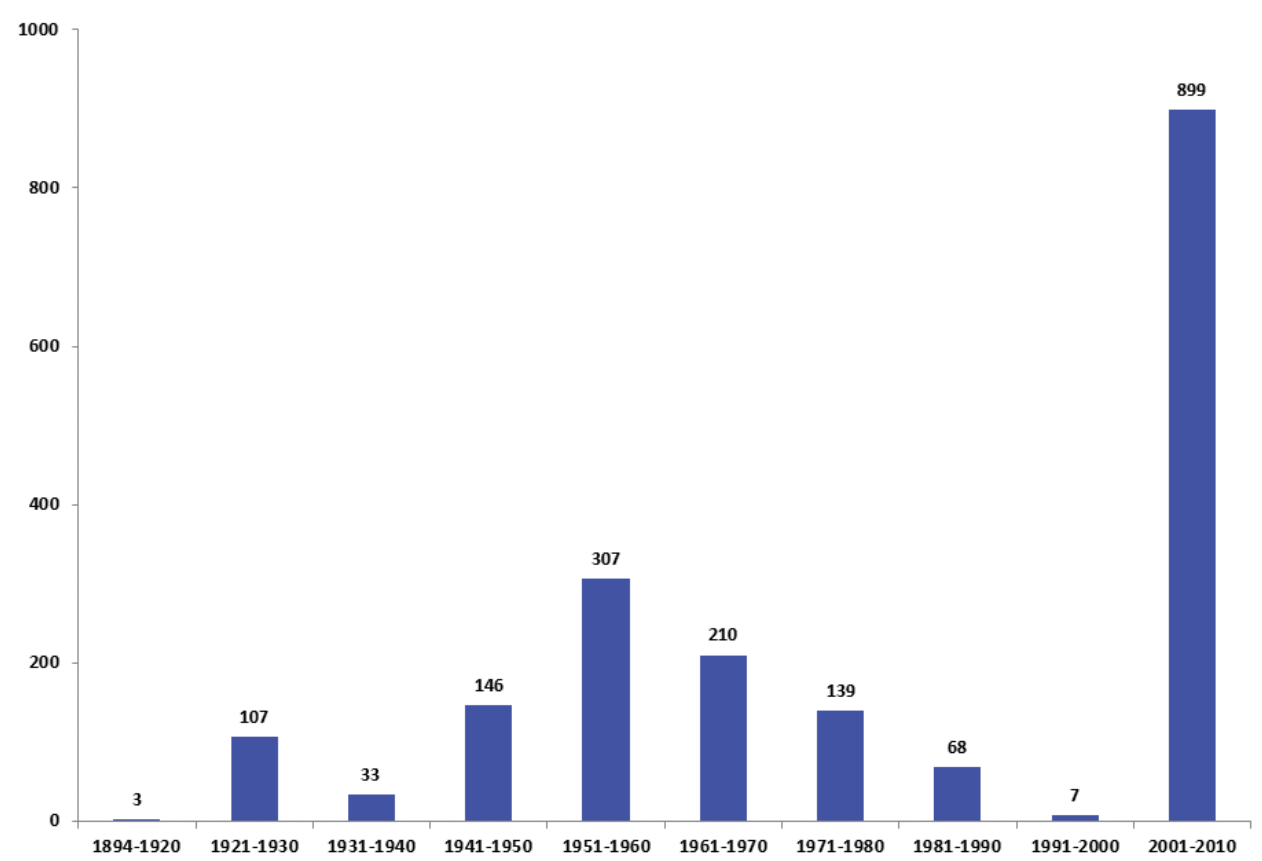

Figure 2. Specimens of Microgastrinae collected in Ottawa by decade (1894-2010). Data based on CNC holdings only.

that southern localities in the United States, when thoroughly sampled and studied, should have higher species totals. But Ottawa presently is the biodiversity hotspot for Microgastrinae in North America, a result of its habitat diversity and the relatively comprehensive taxonomic studies done on its fauna.

The Ottawa microgastrines we studied were collected from 1894-2010 (Figure 2), with most of the specimens collected either from 1945-1975 or 2007-2008. More recent material (2012-2016) is presently being prepared and will be included in future updates of the current species list.

Seasonal distribution of the specimens in general (Figure 3) shows several activity peaks: 1) spring (second half of May to second half of June), 2) a larger peak in summer (late July to early August), and 3) a smaller peak in early fall (September). This pattern is somewhat similar to that found in another intensively studied Canadian locality (Churchill, Manitoba, at $59^{\circ} \mathrm{N}$ ), although there were only two peaks in that more northern area, due to its shorter season (Fernandez-Triana et al. 2011).

The general pattern varies depending on the species. Among the eight most common species, five show only a single peak of abundance, three in summer (Glyptapanteles militaris, Cotesia atalantae and Hygroplitis melligaster), and two in the fall (Apanteles nephoptericis and Glyptapanteles pallipes). Two species have two peaks, Pholetesor ornigis in late spring and late summer, and Hypomicrogaster zonaria in early summer and early fall. Microplitis varicolor has three peaks in spring, summer and fall (Fig. 4). 


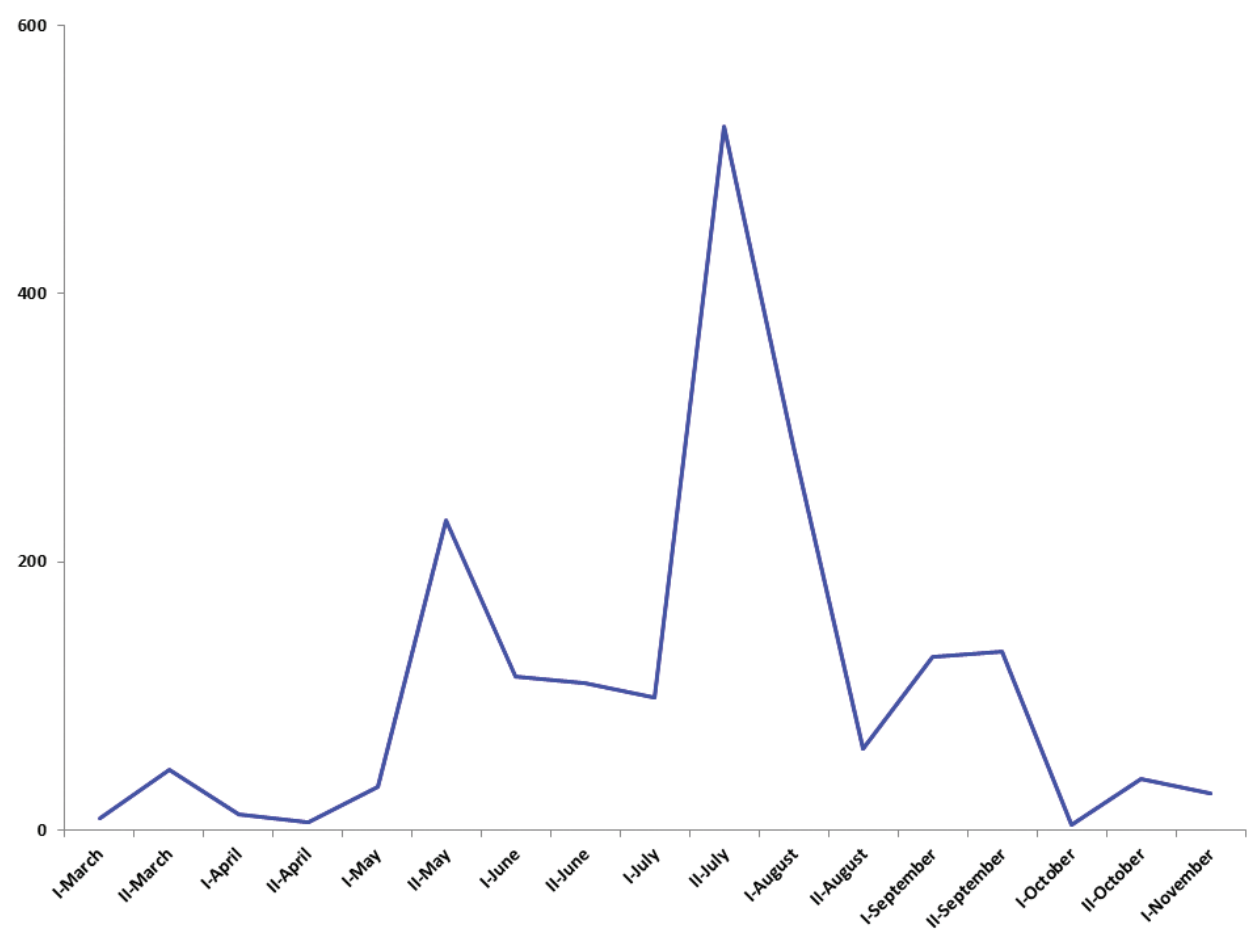

Figure 3. Specimens of Microgastrinae collected in Ottawa bi-weekly (March-November), based on CNC holdings only (1894-2010).

As more collected material becomes available, a better picture of species seasonality will be obtained.

Species of Microgastrinae are parasitoids exclusively of Lepidoptera (Whitfield 1997). There are at least 2,064 species of Lepidoptera already recorded from Ottawa (D. Lafontaine, in lit. 2016, Lafontaine 1997; http://www.acleris.com/dls/habitatdiversity.html), with the actual number probably approaching 2,200 species (D. Lafontaine, pers. com.). A literature search shows that 72 of the Microgastrinae species found in Ottawa have been, at some point, recorded parasitizing at least 554 Lepidoptera species (based on data compiled by Yu et al. 2012).

However, it must be pointed that some of those lepidopterans do not actually occur in Ottawa, and some of the reported host/parasitoid associations are likely to be incorrect (see Shaw et al. 2009 for a discussion and examples that demonstrate the pitfalls of using compilations of host/parasitoid abstracted uncritically from the literature). Our knowledge of the association between lepidopteran caterpillars and their microgastrine parasitoids at the local and/or regional level is still very incomplete, and many more studies are needed before some patterns can be reliably established. Thus, in this paper we do not analyze further the relationships between Microgastrinae and Lepidoptera in Ottawa.

Evidence from recent studies in Area de Conservacion Guanacaste (ACG), Costa Rica, comprising 11,000+ Lepidoptera and 1,100+ Microgastrinae species (e.g., Janzen 

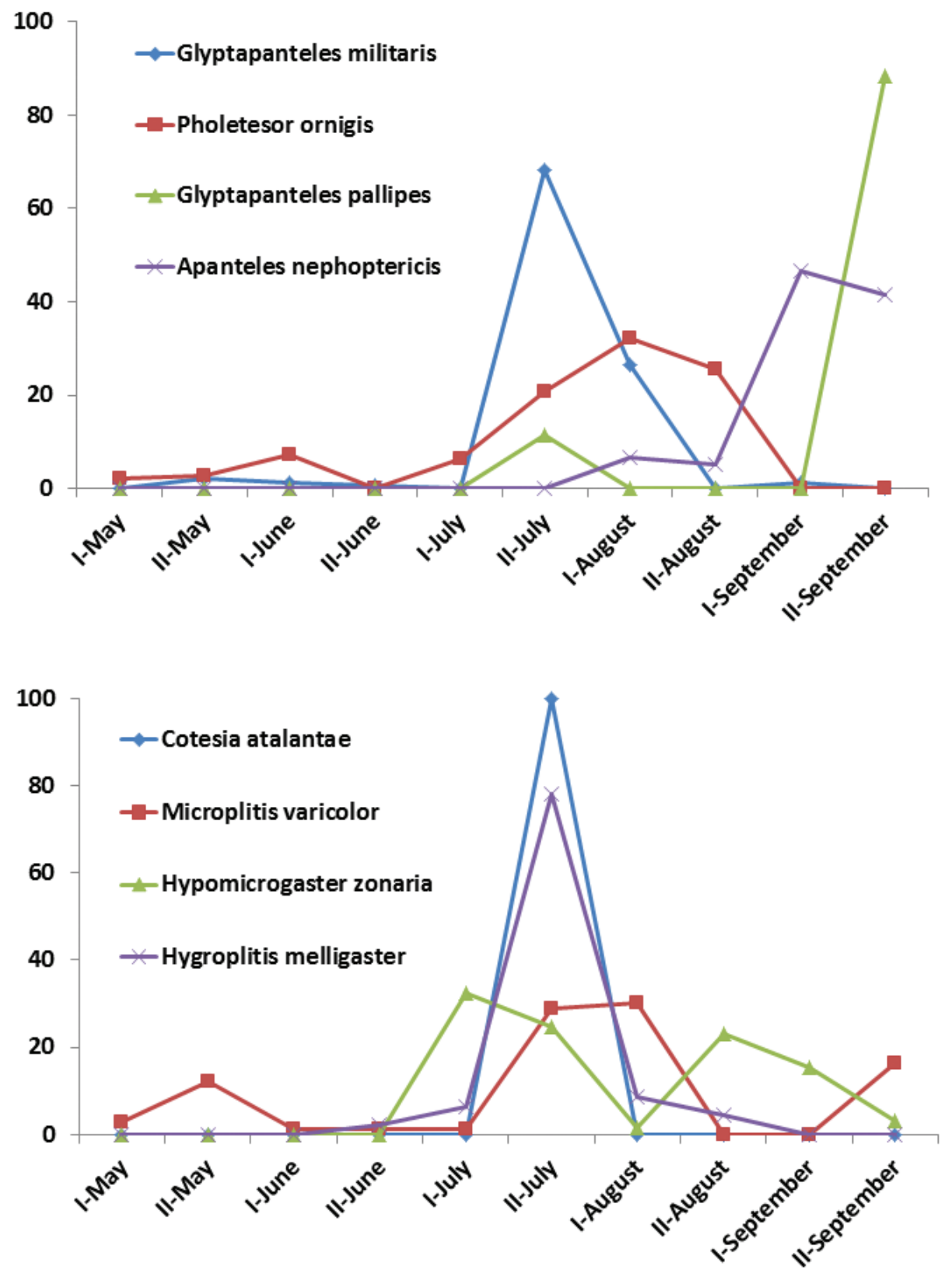

Figure 4. Bi-weekly (March-November) abundance of the eight most common species of Microgastrinae collected in Ottawa, based on CNC holdings only (1894-2010).

and Hallwachs 2016) shows that microgastrine wasps are more specialized (=host specific) than previously thought. Most species have been found parasitizing one/few species of Lepidoptera which are usually phylogenetically related or, alternatively, related ecologically 
in the niche they occupy (e.g., Fernandez-Triana et al. 2014d). At present there is no comprehensively enough information in North America to compare, but the available (more limited) data suggests that it might well be the same situation as in the tropics. Based on the currently verified and available data, the ratio between species of Lepidoptera $(2,064)$ and Microgastrinae (158) in Ottawa stands at 13.1, very similar to the ratio found in ACG and other regions of world (Fernandez-Triana 2010, Rodriguez et al. 2013, Janzen and Hallwachs 2016). The significance of the Lepidoptera/Microgastrinae ratio has been discussed further in Rodriguez et al. (2013).

\section{Annotated checklist of Microgastrinae from Ottawa, Canada}

\section{Alphomelon winniewertzae Deans, 2003}

Fig. 5

\section{Distribution. NEA, NEO.}

Notes. The status of this species as a potential member of the Species Candidate Lists of The Committee on the Status of Endangered Wildlife in Canada (COSEWIC, http://www.cosewic.gc.ca/) was assessed by Fernandez Triana (2014).

Material examined. Quebec, Old Chelsea, 45.503548 -75.797963, 351m, 11.viii.1965, J. R. Vockeroth, Voucher Code: CNCHYM00025.

\section{Apanteles baldufi Muesebeck, 1968}

Distribution. NEA.

Material examined. Ontario, Blackburn, 45.430272 -75.563017, 15.vi.1942, J. McDunnough, Voucher Code: MIC000024; 20.vi.1931, G.S. Walley, Voucher Code: MIC000020; Dirleton, Ont, 45.495069 -76.142986, 25.vi.1963, G.S. Walley, Voucher Code: CNC280541.

\section{Apanteles canarsiae Ashmead, 1898}

Distribution. NEA.

Material examined. Quebec, Wrightville, 45.438108 -75.743308, 23.vi.1939, F.I.S., Voucher Code: MIC000032.

Apanteles carpatus (Say, 1836)

Distribution. Cosmopolitan. 
Material examined. Ontario, Ottawa, city garden, $45.356-75.707$, 10.viii.2007, H. Goulet, Voucher Code: CAM0036, CAM0042; 13.vii.2007, H. Goulet, Voucher Code: CAM0020, CAM0021, CAM0058, CAM0063; 19.ix.2007, H. Goulet, Voucher Code: CAM0023; 23.vii.2007, H. Goulet, Voucher Code: CAM0046, CAM0049, CAM0053; 30.vii.2007, H. Goulet, Voucher Code: CAM0026, CAM0117; 8.ix.2007, H. Goulet, Voucher Code: CAM0104; Ottawa, 45.356083 -75.706933, 12.iii.1952, J.R. Vockeroth, Voucher Code: CNC280555; 45.3825 -75.7137, 15.ix.1955, J. R. Vockeroth, Voucher Code: MIC000035; 45.382500 -75.713700, 8.v.1928, C. Twinn, Voucher Code: MIC000033; 45.406631 -75.701407, 4.iii.1952, J.R. Vockeroth , Voucher Code: CNC474712; v.1952, J.R. Vockeroth, Voucher Code: CNC474713.

\section{Apanteles conanchetorum Viereck, 1917}

\section{Distribution. NEA.}

Material examined. Ontario, Aylmer West, 45.400000 -75.850000, 20.vii.1972, Voucher Code: MIC000060; Ottawa, city garden, 45.356 -75.707, 1.ix.2007, H. Goulet, Voucher Code: CAM0147, CAM0159, CAM0160; 10.viii.2007, H. Goulet, Voucher Code: CAM0085; 19.ix.2007, H. Goulet, Voucher Code: CAM0005; 30.vii10.viii.2007, H. Goulet, Voucher Code: CAM1013; Woodlawn, 45.375 -76.083, 6.viii.2008, L. Masner, Voucher Code: MIC000600.

\section{Apanteles crassicornis (Provancher, 1886)}

Fig. 6

Distribution. NEA.

Material examined. Ontario, Merivale, 45.325948 -75.719082, 17.viii.1930, J.J. de Gryse, Voucher Code: MIC000071; Ottawa, city garden, 45.356083 -75.706933, 1-19.ix.2007, H. Goulet, Voucher Code: CNC280561; 10.viii-1.ix.2007, H. Goulet, Voucher Code: MIC000067, MIC000069.

\section{Apanteles depressariae Muesebeck, 1931}

\section{Distribution. NEA.}

Material examined. Ontario, Ottawa, Hogs Back, 45.372817 -75.698000, 1117.vii.1951, C.D. Miller, Voucher Code: MIC000074; 45.372865 -75.697930, 11-16.vii.1951, Miller, C.O., Voucher Code: CNC280565; Quebec, Kirk’s Ferry, 45.541575 -75.817381, 24.v.1950, Bierne, Voucher Code: MIC000075. 

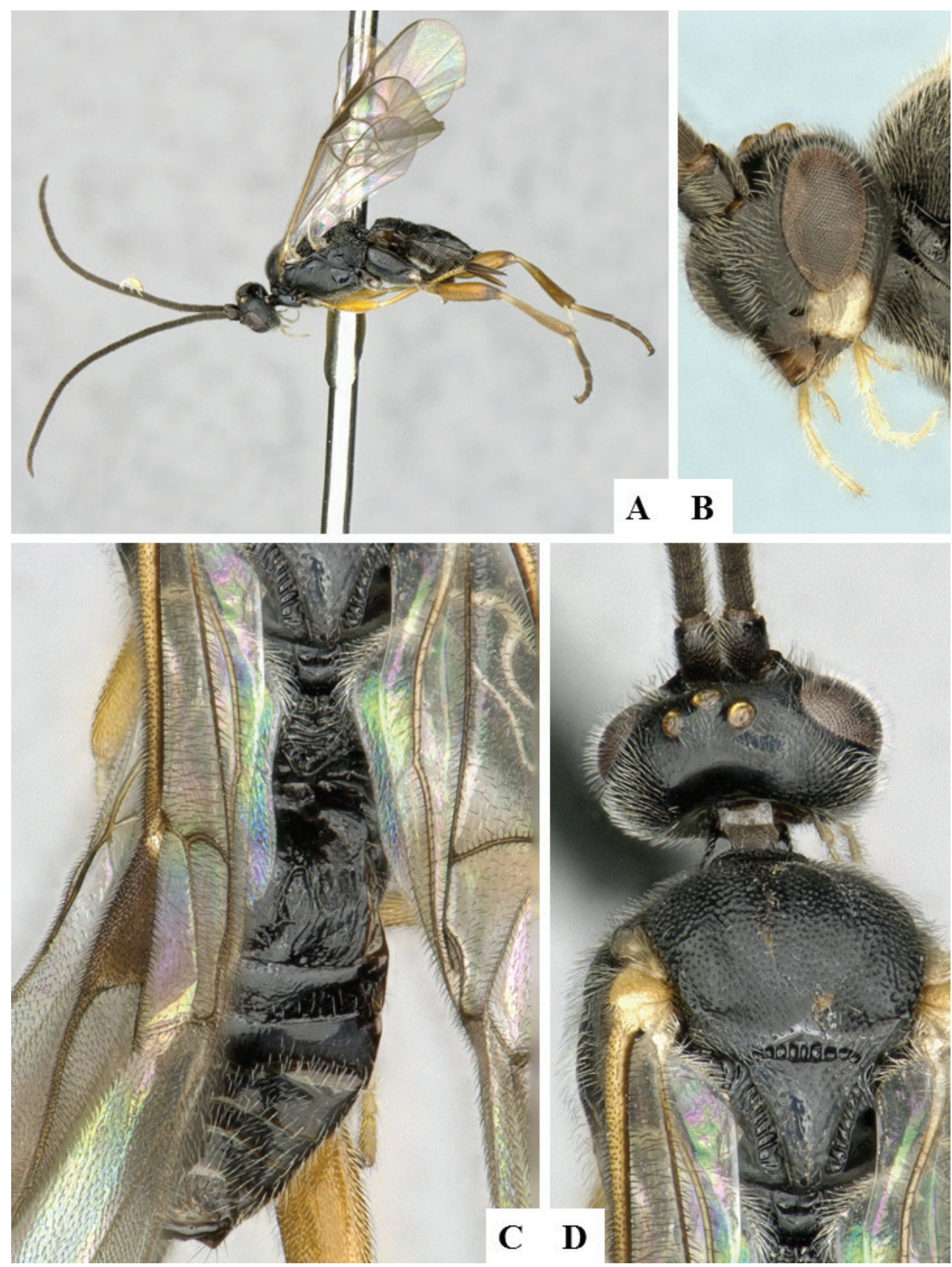

Figure 5. Alphomelon winniewertzae. A- Habitus, lateral B Head, lateral C Metasoma, dorsal D Head and mesosoma (partially), dorsal. 

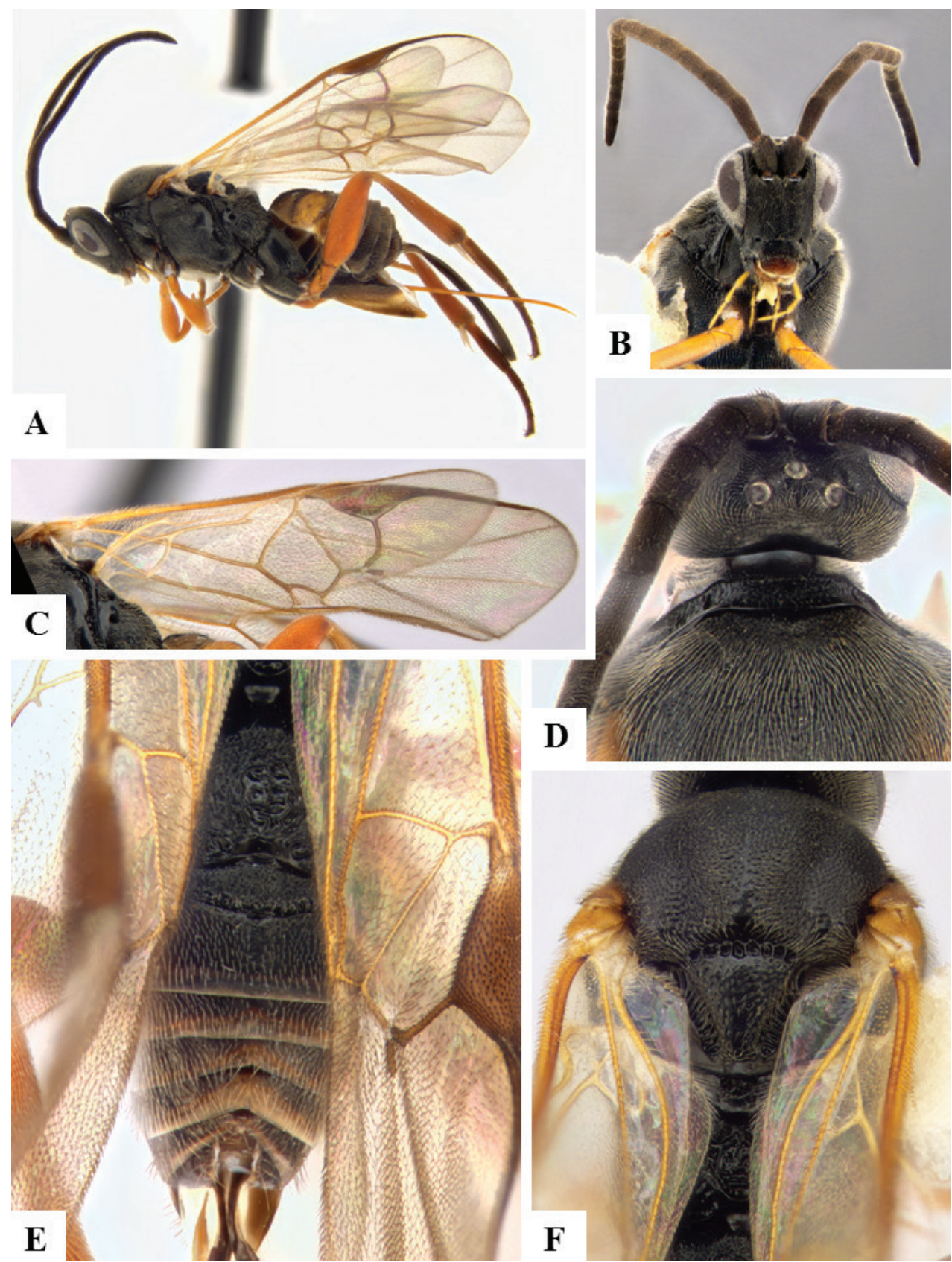

Figure 6. Apanteles crassicornis. A Habitus, lateral B Head, frontal C Wings D Head and mesosoma (partially), dorsal E Metasoma, dorsal F Mesosoma, dorsal. 
Apanteles epinotiae Viereck, 1912

Distribution. NEA.

Material examined. Ontario, Ottawa, 45.406631 -75.701407, 22.vii.1954, W.R.M. Mason, Voucher Code: MIC000094.

\section{Apanteles forbesi Viereck, 1910}

Distribution. NEA.

Material examined. Ontario, Blackburn, 45.430270 -75.563015, 9.vi.1939, O. Peck, Voucher Code: MIC000100.

\section{Apanteles fumiferanae Viereck, 1912}

Distribution. NEA, PAL.

Material examined. Ontario, Ottawa, $45.356083-75.706933$, 23.vi.1945, F.I.S, Voucher Code: CNC280597; 45.3825-75.7137, 23.vi.1945, Voucher Code: MIC000282; South March, 45.348507 -75.923123, 17.vi.1949, Voucher Code: MIC000272.

\section{Apanteles jenniferae Fernandez-Triana, 2010}

Fig. 7

Distribution. NEA.

Material examined. Ontario, Galetta, 45.433392 -76.250133, 28.vi.1951, Voucher Code: CNCHYM07234; Quebec, Old Chelsea, 45.503548 -75.797963, 19.vi.1940, Voucher Code: CNCHYM07232; Tenaga, 45.531020 -75.798359, 1.vii.1940, Voucher Code: CNCHYM07233.

Apanteles laricellae Mason, 1959

Fig. 8

Distribution. NEA.

Material examined. Ontario, Kemptville Rideau, 45.016409 -75.646449, 21.v.1957, F.I.S., Voucher Code: MIC000120; 21.v.1957, F.I.S., Voucher Code: CNC474716; Mer Bleue, 45.393578 -75.512128, 1.vi.1960, C.D. Miller, Voucher Code: CNC280644; 45.393585 -75.512138, 6.vi.1960, C.D. Miller, Voucher Code: MIC000121; 7.vi.1960, C.D. Miller, Voucher Code: CNC474717; 45.393593 -75.512138, 1.vi.1960, C.D. Miller, Voucher Code: CNCHYM00142. 
Apanteles morrisi Mason, 1974

Fig. 9

Distribution. NEA, PAL.

Material examined. Ontario, North Gower to Smith Falls, $1 \mathrm{~km} \mathrm{~N}$ of Rd 6 \& Montague Bdy Rd, 45.033 -75.9, 15.vi.2004, Bennett \& Barnes, Voucher Code: HYM00001061.

\section{Apanteles nephoptericis (Packard, 1864)}

\section{Distribution. NEA.}

Material examined. Ontario, Ottawa, 45.356083 -75.706933, 15.viii.1955, W. R. M. Mason, Voucher Code: CNC280686; 45.3825 -75.7137, 1.ix.1955, W. R. M. Mason, Voucher Code: CNCHYM00172; 15.viii.1955, W. R. M. Mason, Voucher Code: CNCHYM00173, MIC000151; 45.406631 -75.701407, 6.ix.1954, R. Lambert, Voucher Code: MIC000154, MIC000155; 7.ix.1955, W.R.M. Mason, Voucher Code: MIC000152, MIC000153; 1.ix.1955, W.R.M. Mason, Voucher Code: CNC474776-CNC474783; 15.viii.1955, W.R.M. Mason, Voucher Code: CNC474788, CNC474789, CNC474790; 17.viii.1955, W.R.M. Mason, Voucher Code: CNC474784, CNC474785, CNC474786, CNC474787; 20.ix.1955, W.R.M. Mason, Voucher Code: CNC474718-CNC474748, CNC474791; 6.ix.1955, W.R.M. Mason, Voucher Code: CNC474763, CNC474764, CNC474765, CNC474766, CNC474767, CNC474768, CNC474769, CNC474770, CNC474771, CNC474772, CNC474773, CNC474774, CNC474775; 7.ix.1955, W.R.M. Mason, Voucher Code: CNC474749, CNC474750, CNC474751, CNC474752, CNC474753, CNC474754, CNC474755, CNC474756, CNC474757, CNC474758, CNC474759, CNC474760, CNC474761, CNC474762.

\section{Apanteles petrovae Walley, 1937}

Fig. 10

Distribution. NEA, PAL.

Material examined. Ontario, Constance Bay, 45.486218 -76.073461, 14.v.1946, F.I.S. No.4-19, Voucher Code: CNC474792; 45.486248 -76.073504, 16.v.1934, G.S. Walley, Ottawa, city garden, $45.3561-75.7069$, 5.v-5.vi.2008, H. Goulet, Voucher Code: CAM1000, CAM1002, CAM1006, CAM1008, CAM1009; 45.3561 -75.707, 26.vi.2007, H. Goulet, Voucher Code: CAM0073; 30.v.2007, H. Goulet, Voucher Code: CAM0019. 


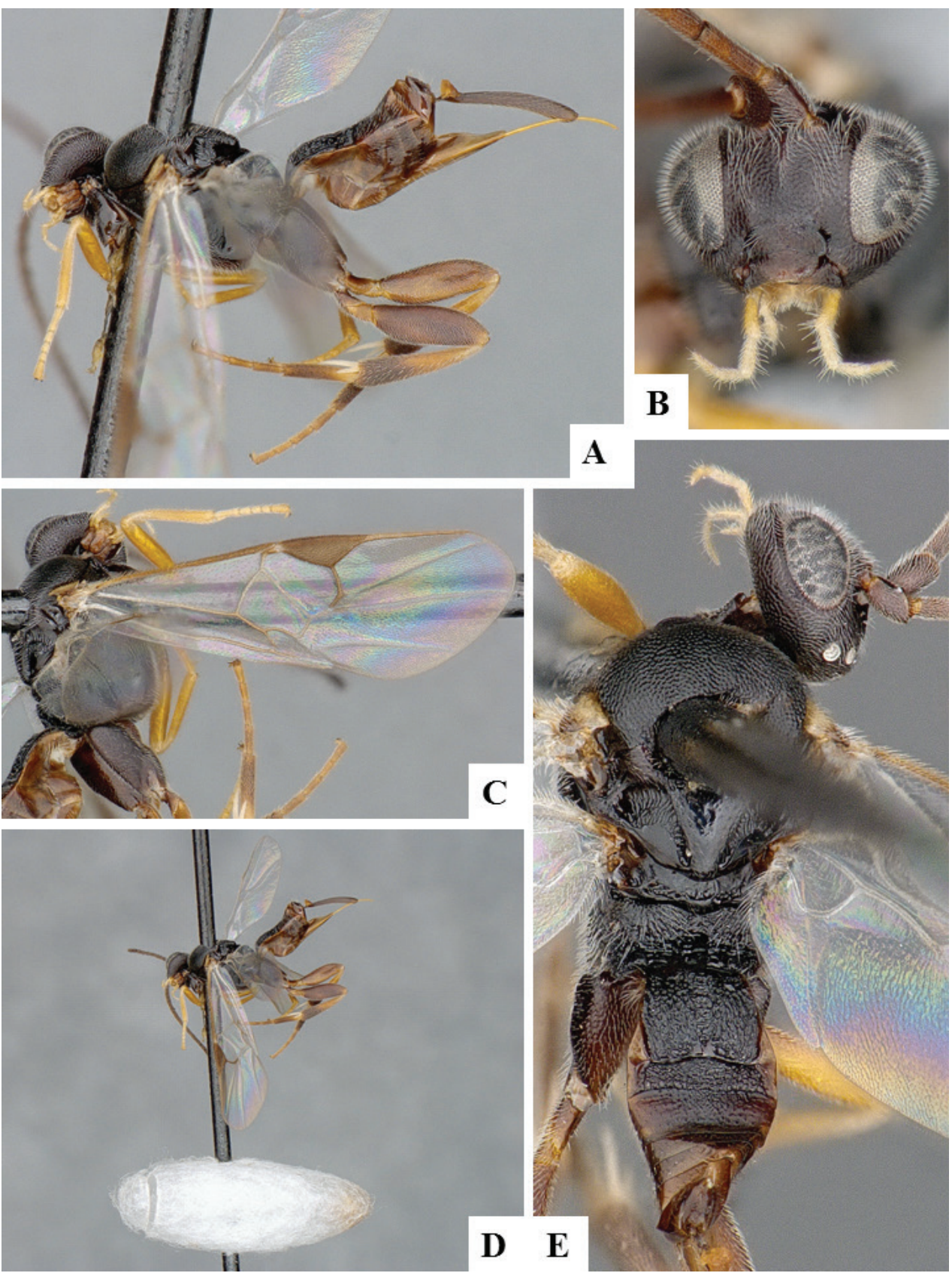

Figure 7. Apanteles jenniferae. A Habitus, lateral B Head, frontal C Wings D Adult wasp and its cocoon E Habitus, dorsal. 


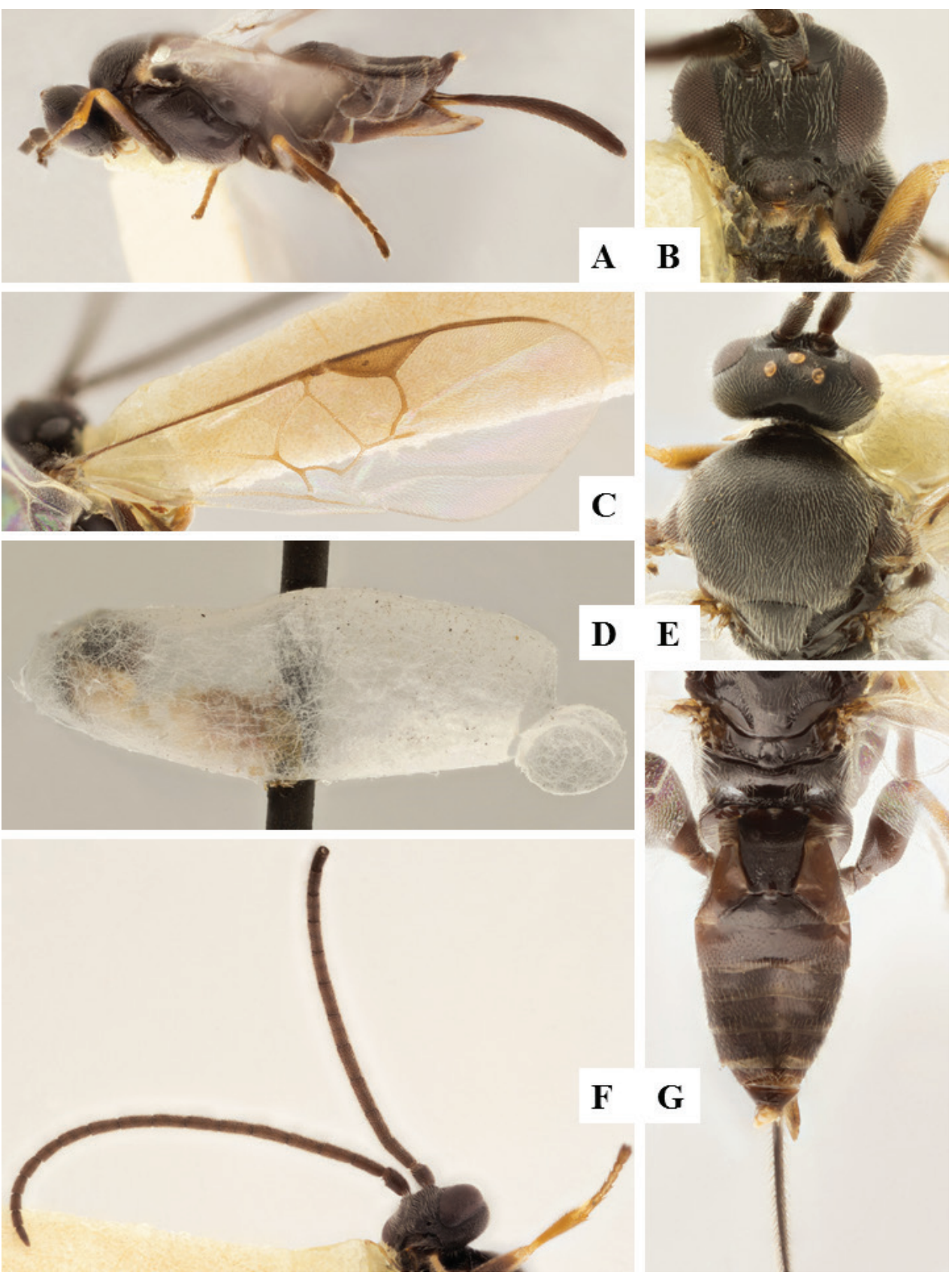

Figure 8. Apanteles laricellae. A Habitus, lateral B Head, frontal C Wings D Head and mesosoma (partially), dorsal E Metasoma, lateral F Metasoma, dorsal F Mesosoma, dorsal. 


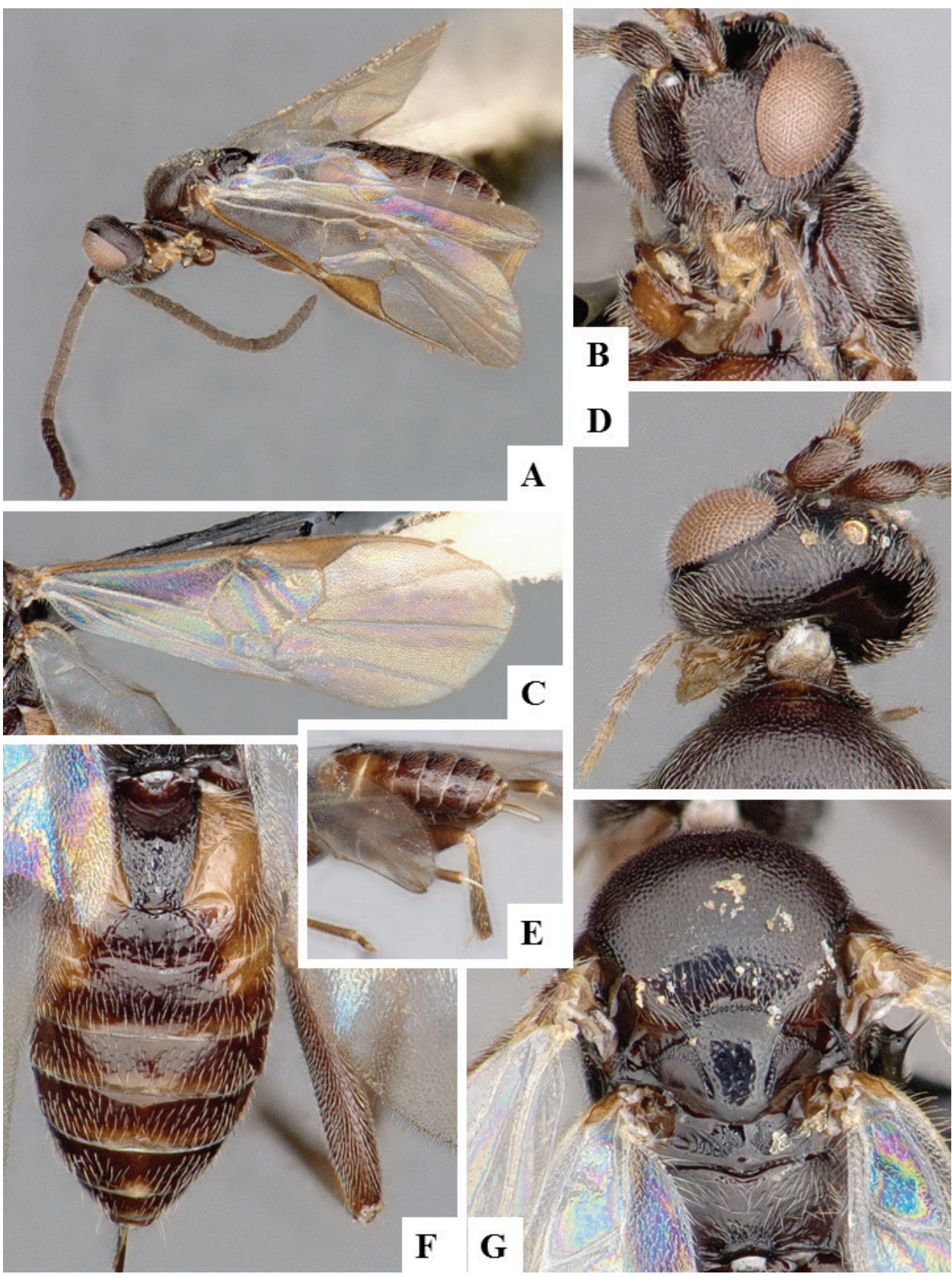

Figure 9. Apanteles morrisi. A Habitus, lateral B Head, frontal C Wings D Head and mesosoma, dorsal E Metasoma dorsal F Head and antenna, dorsal. 

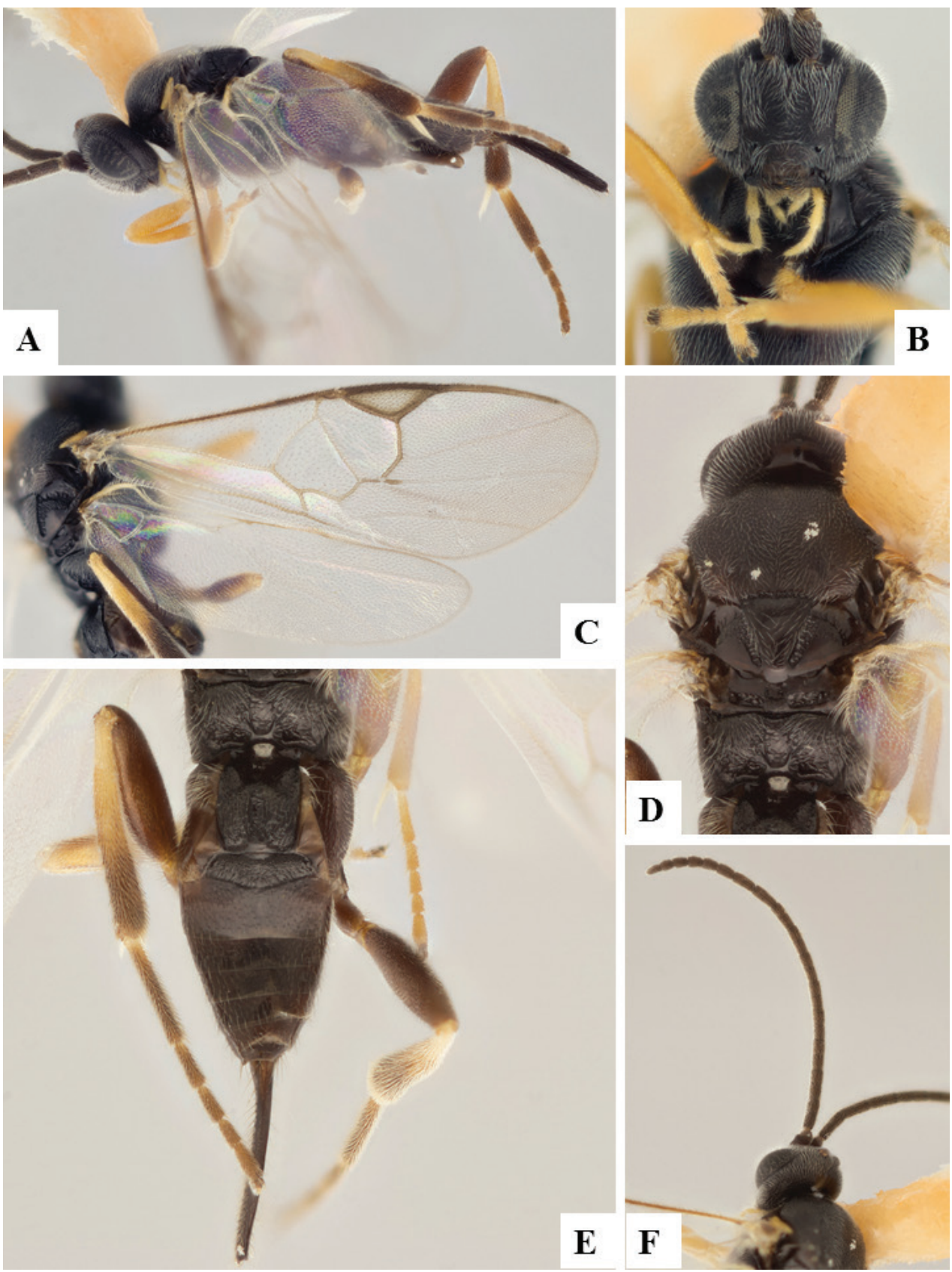

Figure 10. Apanteles petrovae. A Habitus, lateral B Head, frontal C Wings D Cocoon E Head and mesosoma (partially), dorsal F Head and antenna, ventrally $\mathbf{G}$ Metasoma, dorsal. 
Apanteles polychrosidis Viereck, 1912

Fig. 11

\section{Distribution. NEA.}

Material examined. Quebec, Aylmer, 45.4 -75.85, 10.vii.2004, S. Laplante, Voucher Code: HYM00001646.

\section{Apanteles jft01}

\section{Distribution. NEA.}

Notes. This species corresponds in BOLD to BIN BOLD:AAA6373, with specimens found across Canada and US.

Material examined. Ontario, $5 \mathrm{~km} \mathrm{NW}$ of Almonte, Hwy 49, Burnt Land, Alvar Prov. Park, Almonte, 45.2549 -76.14, 29.v.2008, Goulet \& Fernandez, Voucher Code: CAM0353, CAM0357, CAM0361, CAM0364; Ottawa, city garden, 45.3561 -75.707, 2.vii.2007, H. Goulet, Voucher Code: CAM0128; 30.v.2007, H. Goulet, Voucher Code: CAM0012, CAM0017; Woodlawn, 45.375 -76.083, 6.viii.2008, L. Masner, Voucher Code: MIC000599, MIC000601.

\section{Apanteles jft04}

Distribution. NEA.

Material examined. Ontario, Ottawa, city garden, 45.3561 -75.707, 15.vii.2007, H. Goulet, Voucher Code: CAM0123.

\section{Apanteles jft09}

\section{Distribution. NEA.}

Notes. This species corresponds in BOLD to BIN BOLD:AAA8872, with all specimens collected in southern Ontario.

Material examined. Ontario, Ottawa, city garden, 45.3561 -75.7069, 28.vii.2009, L. Masner, Voucher Code: CNCH1024; 30.vii-10.viii.2007, H. Goulet, Voucher Code: CAM1014; 45.3561 -75.707, 1.ix.2007, H. Goulet, Voucher Code: CAM0080, CAM0156, CAM0162; 10.viii.2007, H. Goulet, Voucher Code: CAM0032, CAM0035, CAM0037; 13.vii.2007, H. Goulet, Voucher Code: CAM0064; 16.vi.2007, H. Goulet, Voucher Code: CAM0111; 19.ix.2007, H. Goulet, Voucher Code: CAM0006, CAM0010; 23.vii.2007, H. Goulet, Voucher Code: CAM0045, CAM0047, CAM0052; 26.vi.2007, H. Goulet, Voucher Code: CAM0066, CAM0067; 30.vii.2007, H. Goulet, Voucher Code: CAM0028; 8.ix.2007, H. Goulet, Voucher Code: CAM0107; 45.356100 -75.707000, 8.ix.2007, H. Goulet, Voucher Code: CAM0102. 

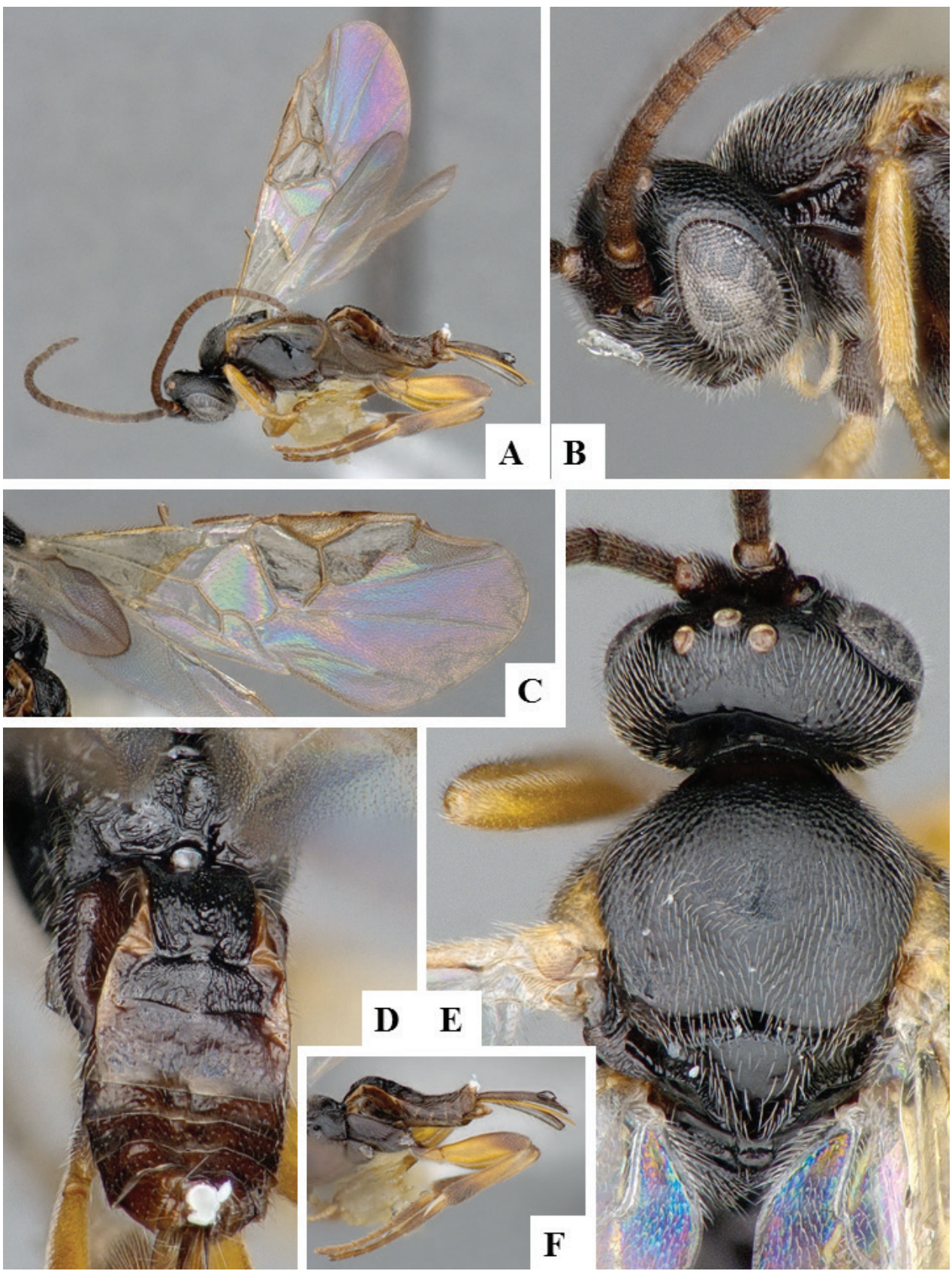

Figure II. Apanteles polychrosidis. A Habitus, lateral B Head, lateral C Wings D Metasoma, dorsal E Head and mesosoma (partially), dorsal F Metasoma, lateral.

\section{Apanteles jft10}

Distribution. NEA. 
Notes. This species corresponds in BOLD to BIN BOLD:AAA8871, with all specimens collected in North America.

Material examined. Ontario, $5 \mathrm{~km} \mathrm{NW}$ of Almonte, Hwy 49, Burnt Land, Alvar Prov. Park, Almonte, 45.2549 -76.14, 29.v.2008, Goulet \& Fernandez, Voucher Code: CAM0334, CAM0336, CAM0337, CAM0338, CAM0339, CAM0354, CAM0358, CAM0359.

\section{Apanteles jft12}

Distribution. NEA.

Notes. This species corresponds in BOLD to BIN BOLD:AAB8748, with all specimens collected in Canada.

Material examined. Ontario, Island Park, Ottawa, 45.407035 -75.747564, 14.x.2008, L. Masner, Voucher Code: MIC000603; Ottawa, city garden, 45.3561 -75.7069, 24-30.v.2007, H. Goulet, Voucher Code: CAM0872; 45.3561 -75.707, 26.vi.2007, H. Goulet, Voucher Code: CAM0068, CAM0069, CAM0070, CAM0071; 30.v.2007, H. Goulet, Voucher Code: CAM0018; 8.vi.2007, H. Goulet, Voucher Code: CAM0065.

\section{Apanteles jft14}

Distribution. NEA.

Notes. This species corresponds in BOLD to BIN BOLD:AAD2306, with all specimens collected in eastern Canada.

Material examined. Ontario, Ottawa, city garden, $45.3561-75.707$, 1.ix.2007, H. Goulet, Voucher Code: CAM0043, CAM0090, CAM0148.

\section{Apanteles jft15}

\section{Distribution. NEA.}

Notes. This species corresponds in BOLD to BIN BOLD:ACL9543, with all specimens collected in North America.

Material examined. Ontario, Ottawa, city garden, 45.3561 -75.7069, 1-19. ix.2007, H. Goulet, Voucher Code: CAM0871; 45.3561 -75.707, 19.ix.2007, H. Goulet, Voucher Code: CAM0003.

\section{Apanteles jft25}

Distribution. NEA. 
Notes. This species corresponds in BOLD to BIN BOLD:AAA9844, with all specimens collected found in Ottawa.

Material examined. Ontario, Ottawa, city garden, 45.3561 -75.707, 1.ix.2007, H. Goulet, Voucher Code: CAM0077, CAM0078, CAM0079, CAM0081, CAM0086, CAM0088, CAM0089, CAM0152, CAM0155, CAM0157, CAM0158, CAM0164, CAM0165, CAM0167, CAM0168; 10.viii.2007, H. Goulet, Voucher Code: CAM0029, CAM0031, CAM0033, CAM0034, CAM0038, CAM0040, CAM0116; 13.vii.2007, H. Goulet, Voucher Code: CAM0059; 15.vii.2007, H. Goulet, Voucher Code: CAM0121, CAM0125; 30.vii.2007, H. Goulet, Voucher Code: CAM0027, CAM0097, CAM0118, CAM0119; 45.356100 -75.707000, 10.viii.2007, H. Goulet, Voucher Code: CAM0041; Woodlawn, 45.375 -76.083, 17.viii.2008, L. Masner, Voucher Code: MIC000602; 8.ix.2008, L. Masner, Voucher Code: MIC000604.

\section{Apanteles jft26}

\section{Distribution. NEA.}

Notes. This species corresponds in BOLD to BIN BOLD:AAA8770, with all specimens collected in North America.

Material examined. Ontario, $5 \mathrm{~km} \mathrm{NW}$ of Almonte, Hwy 49, Burnt Land, Alvar Prov. Park, Almonte, 45.2549 -76.14, 29.v.2008, Goulet \& Fernandez, Voucher Code: CAM0363; Ottawa, city garden, 45.3561 -75.707, 1.ix.2007, H. Goulet, Voucher Code: CAM0083; 30.v.2007, H. Goulet, Voucher Code: CAM0014, CAM0016.

\section{Apanteles jft27}

Distribution. NEA.

Notes. This species corresponds in BOLD to BIN BOLD:ACF4409, with all specimens collected in southern Ontario.

Material examined. Ontario, Ottawa, city garden, 45.3561 -75.707, 1.ix.2007, H. Goulet, Voucher Code: CAM0150; 10.viii.2007, H. Goulet, Voucher Code: CAM0030; 13.vii.2007, H. Goulet, Voucher Code: CAM0055; 15.vii.2007, H. Goulet, Voucher Code: CAM0122, CAM0126; 23.vii.2007, H. Goulet, Voucher Code: CAM0050; Woodlawn, 45.375 -76.083, 8.ix.2008, L. Masner, Voucher Code: MIC000605.

\section{Apanteles jft28}

\section{Distribution. NEA.}

Notes. This species corresponds in BOLD to BIN BOLD:AAF7782, with all specimens collected in eastern Canada. 
Material examined. Ontario, Ottawa, city garden, 45.3561 -75.707, 15.vii.2007, H. Goulet, Voucher Code: CAM0127.

\section{Apanteles jft31}

Distribution. NEA, PAL.

Notes. This species corresponds in BOLD to BIN BOLD:AAB0096, with specimens collected in the Nearctic (Canada, US), and the Palearctic (Russia).

Material examined. Ontario, mixed forest, 45.2347 -75.624, 19-29.vi.2007, A. Bennett, Voucher Code: CAM0533; 7-19.vi.2007, A. Bennett, Voucher Code: CAM0535, CAM0536; Ottawa, city garden, 45.3561 -75.7069, 16-26.vi.2007, H. Goulet, Voucher Code: CAM0898; 24-30.v.2007, H. Goulet, Voucher Code: CAM0873; 45.3561 -75.707, 13.vii.2007, H. Goulet, Voucher Code: CAM0056; 15.vii.2007, H. Goulet, Voucher Code: CAM0124; 16.vi.2007, H. Goulet, Voucher Code: CAM0105, CAM0108, CAM0109, CAM0110, CAM0112; 2.vii.2007, H. Goulet, Voucher Code: CAM0129, CAM0130, CAM0131, CAM0132, CAM0133, CAM0134, CAM0135, CAM0136, CAM0137, CAM0138, CAM0139, CAM0140, CAM0141, CAM0142, CAM0143, CAM0144, CAM0145; 26.vi.2007, H. Goulet, Voucher Code: CAM0075.

\section{Apanteles jft37}

\section{Distribution. NEA.}

Notes. This species corresponds in BOLD to BIN BOLD:AAC3220, with all specimens collected in North America.

Material examined. Ontario, Ottawa, city garden, $45.3561-75.7069$, 23.vii.2009, L. Masner, Voucher Code: CNCH0459; 45.3561 -75.707, 1.ix.2007, H. Goulet, Voucher Code: CAM0082; 13.vii.2007, H. Goulet, Voucher Code: CAM0061; 23.vii.2007, H. Goulet, Voucher Code: CAM0051; 30.vii.2007, H. Goulet, Voucher Code: CAM0098; Woodlawn, 45.375 -76.083, 6.viii.2008, L. Masner, Voucher Code: MIC000598.

\section{Apanteles sp. $1 \mathrm{nr}$ conanchetorum}

\section{Distribution. NEA.}

Notes. This species is morphologically close to Apanteles conanchetorum Viereck, 1917 but DNA barcodes are different (BINS: BOLD:AAC5506 and BOLD:AAC5507) and it has been considered as a separate species by Fernandez-Triana et al. (2014).

Material examined. Ontario, Ottawa city, Slack road, 45.321539 -75.730767, 5.x.2007, L. Masner, Voucher Code: CAM0096. 


\section{Apanteles sp.1 nr ensiger}

\section{Distribution. NEA.}

Notes. The specimens of 'Apanteles ensiger (Say, 1836)' that have rendered DNA barcodes comprise two BINS (BOLD:ACE6783 and BOLD:AAA3764) and have been considered as separate species by Fernandez-Triana et al. (2014).

Material examined. Ontario, $2 \mathrm{~km} \mathrm{SW}$ of Innisville, 45.054942 -76.250619, 26.vi.1991, Sharkey \& Read, Voucher Code: GOU0303; 5 km NW of Almonte, Hwy 49, Burnt Land, Alvar Prov. Park, Almonte, 45.255 -76.14, 29.v.2008, Goulet \& Fernandez, Voucher Code: CAM0335, CAM0348; Blackburn, 45.436469 -75.549278, 9.vi.1939, O. Peck, Voucher Code: MIC000083; Britannia, Ottawa, 45.362878 -75.794003, 20.vi.1947, G. Shewell, Voucher Code: MIC000082; mixed forest near Manotick, Ottawa, 45.235 -75.624, 19-29.vi.2007, A. Bennett, Voucher Code: CAM0521, CAM0522; 29.vi-16.vii.2007, A. Bennett, Voucher Code: CAM0525, CAM0526, CAM0527, CAM0528, CAM0529, CAM0530, CAM0531; 7-19.vi.2007, A. Bennett, Voucher Code: CAM0523, CAM0524; North Gower to Smith Falls, $1 \mathrm{~km} \mathrm{~N}$ of Rd 6 \& Montague Bdy Rd, 45.033 -75.9, 15.vi.2004, Bennett \& Barnes, Voucher Code: HYM00001003; Ottawa, city garden, 45.356 -75.707, 1.ix.2007, H. Goulet, Voucher Code: CAM0154, CAM0161; 10.viii.2007, H. Goulet, Voucher Code: CAM0114; 13.vii.2007, H. Goulet, Voucher Code: CAM0022; 19.ix.2007, H. Goulet, Voucher Code: CAM0009; 26.vi.2007, H. Goulet, Voucher Code: CAM0074; 30.vii.2007, H. Goulet, Voucher Code: CAM0024, CAM0025; Quebec, Gatineau Park, 45.600556 -76.042647, 15.vi.1977, L. Masner, Voucher Code: CNC474715; 45.600572 -76.042647, 15.vi.1977, L. Masner, Voucher Code: MIC000090.

\section{Apanteles sp. 2 nr ensiger}

\section{Distribution. NEA.}

Notes. The specimens of 'Apanteles ensiger (Say, 1836)' that have rendered DNA barcodes comprise two BINS (BOLD:ACE6783 and BOLD:AAA3764) and have been considered as separate species by Fernandez-Triana et al. (2014).

Material examined. Ontario, $5 \mathrm{~km} \mathrm{NW}$ of Almonte, Hwy 49, Burnt Land, Alvar Prov. Park, Almonte, 45.255 -76.14, 29.v.2008, Goulet \& Fernandez, Voucher Code: CAM0340, CAM0341, CAM0342, CAM0344, CAM0345, CAM0346, CAM0347, CAM0349, CAM0350, CAM0351, CAM0352, CAM0356, CAM0360, MIC000666; Ottawa, city garden, 45.356 -75.707, 1.ix.2007, H. Goulet, Voucher Code: CAM0146, CAM0151, CAM0163, CAM0166; 10.viii.2007, H. Goulet, Voucher Code: CAM0039, CAM0113, CAM0115; 19.ix.2007, H. Goulet, Voucher Code: CAM0007, CAM0008; 23.vii.2007, H. Goulet, Voucher Code: CAM0054; 26.vi.2007, H. Goulet, Voucher Code: CAM0062, CAM0072, CAM0076; 30.v.2007, H. Goulet, Voucher Code: CAM0011, CAM0013; 30.vii.2007, H. Goulet, Voucher 
Code: CAM0120; 6.vii.2009, L. Masner, Voucher Code: CNCH1025; 8.ix.2007, H. Goulet, Voucher Code: CAM0099, CAM0103, CAM0106; 45.356000 -75.707000, 8.ix.2007, H. Goulet, Voucher Code: CAM0101.

\section{Choeras consimilis (Viereck, 1911)}

Fig. 12

\section{Distribution. NEA.}

Material examined. Ontario, Ottawa, $45.3825-75.7137$, 1.ix.1952, J. F. McAlpine, Voucher Code: CNCHYM00278; Quebec, Gatineau Park, 45.600555 -76.042647, 13.vi.1966, D.P. Pielou, Voucher Code: CNC474797; 14.vii.1966, D.P. Pielou, Voucher Code: CNC474794; 15.vi.1965, W.G. Mathewman, Voucher Code: CNC474795; 23.vi.1966, D.P. Pielou, Voucher Code: CNC474793; 4.vii.1966, D.P. Pielou, Voucher Code: CNC474796; 45.60057 -76.042647, 16.vi.1967, W. G. Matthewman, Voucher Code: CNCHYM00272; 22.vi.1966, D. P. Pielou, Voucher Code: CNCHYM00277; 4.vii.1967, W. G. Matthewman, Voucher Code: CNCHYM00279.

\section{Choeras parasitellae (Bouché, 1834)}

Distribution. NEA, PAL.

Notes. This is the first record of this species for the Nearctic region.

Material examined. Ontario, Ottawa, city garden, 45.3561 -75.7069, 16-26. vi.2007, H. Goulet, Voucher Code: CAM0908; 5.vi-2.vii.2008, H. Goulet, Voucher Code: CAM0906.

\section{Choeras jft25}

\section{Distribution. NEA.}

Notes. This species corresponds in BOLD to BIN BOLD:AAD7965, with all specimens collected in southern Ontario.

Material examined. Ontario, mixed forest, 45.235 -75.624, 19-29.vi.2007, A. Bennett, Voucher Code: CAM0565.

\section{Choeras jft26}

\section{Distribution. NEA.}

Notes. This species corresponds in BOLD to BIN BOLD:AAD7964, with all specimens collected in Canada.

Material examined. Ontario, mixed forest, 45.235 -75.624, 7-19.vi.2007, A. Bennett, Voucher Code: CAM0564. 


\section{Choeras jft34}

\section{Distribution. NEA.}

Notes. This species corresponds in BOLD to BIN BOLD:AAD7963, with all specimens collected in eastern Canada.

Material examined. Ontario, mixed forest, 45.235 -75.624, 29.vi-16.vii.2007, A. Bennett, Voucher Code: CAM0566; Ottawa, city garden, 45.356 -75.707, 1-19. ix.2007, H. Goulet, Voucher Code: CAM0914; 1.ix.2007, H. Goulet, Voucher Code: CAM0084; 10.viii-1.ix.2007, H. Goulet, Voucher Code: CAM0913; 26.vi13.vii.2007, H. Goulet, Voucher Code: CAM0916, CAM0918; Woodlawn, 45.375 -76.083, 6.viii.2008, L. Masner, Voucher Code: MIC000624, MIC000625.

\section{Clarkinella canadensis Mason, 1981}

Fig. 13

\section{Distribution. NEA.}

Notes. This species is only known from the type locality (Ottawa), where it has been rarely collected. The status of this species as a potential member of the Species Candidate Lists of COSEWIC was assessed by Fernandez Triana (2014).

Material examined. Ontario, Ottawa, city garden, $45.356100-75.707000$, 30.vii.2007, H. Goulet, Voucher Code: CAM0263; 8.ix.2007, H. Goulet, Voucher Code: CAM0262; Ottawa, 45.356083 -75.706933, 28.vii.1959, S.M. Clark, 45.406631 -75.701407, 28.vii.1959, S.M. Clark, Voucher Code: CNC15769.

\section{Cotesia acronyctae (Riley, 1871)}

Fig. 14

Distribution. NEA.

Material examined. Ontario, Ottawa, 45.406631 -75.701407, 4.x.1941, F.I.S., Voucher Code: CNC474798, CNC474799.

\section{Cotesia atalantae (Packard, 1881)}

Fig. 15

\section{Distribution. NEA.}

Material examined. Ontario, 6mi. W Richmond, 45.193269 -75.961153, 27.vii.1973, J.E.H. Martin, Voucher Code: CNC474807, CNC474808, CNC474809, CNC474810, CNC474811, CNC474812, CNC474813, CNC474814, CNC474815, CNC474816, CNC474817, CNC474818, CNC474819, CNC474820, CNC474821, CNC474822, CNC474823, CNC474824, CNC474825, CNC474826, CNC474827, CNC474828, CNC474829, CNC474830, CNC474831, CNC474832, CNC474833, 


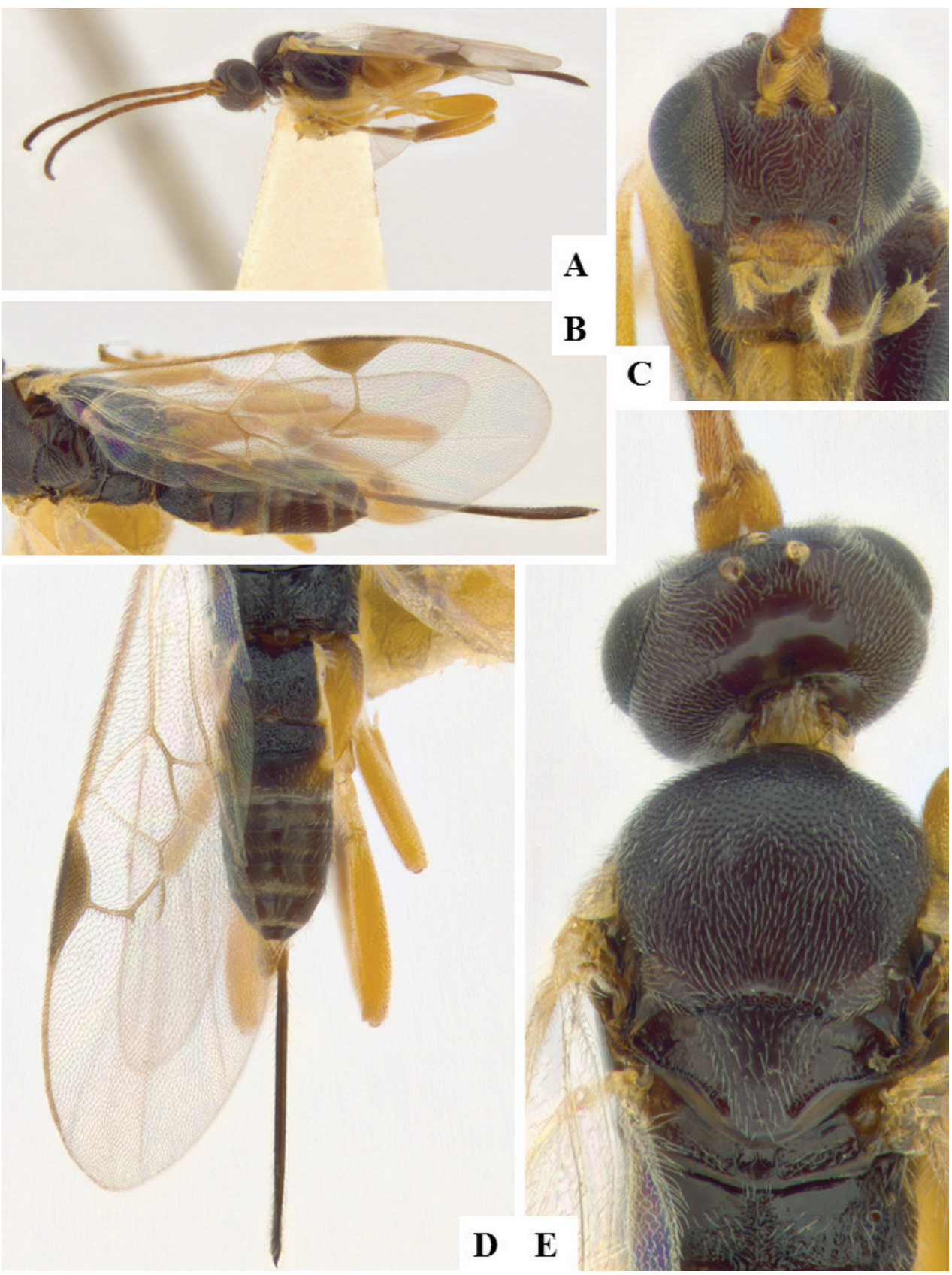

Figure 12. Choeras consimilis. A Habitus, lateral B Wings C Head, frontal D Metasoma dorsal F Head and mesosoma, dorsal. 

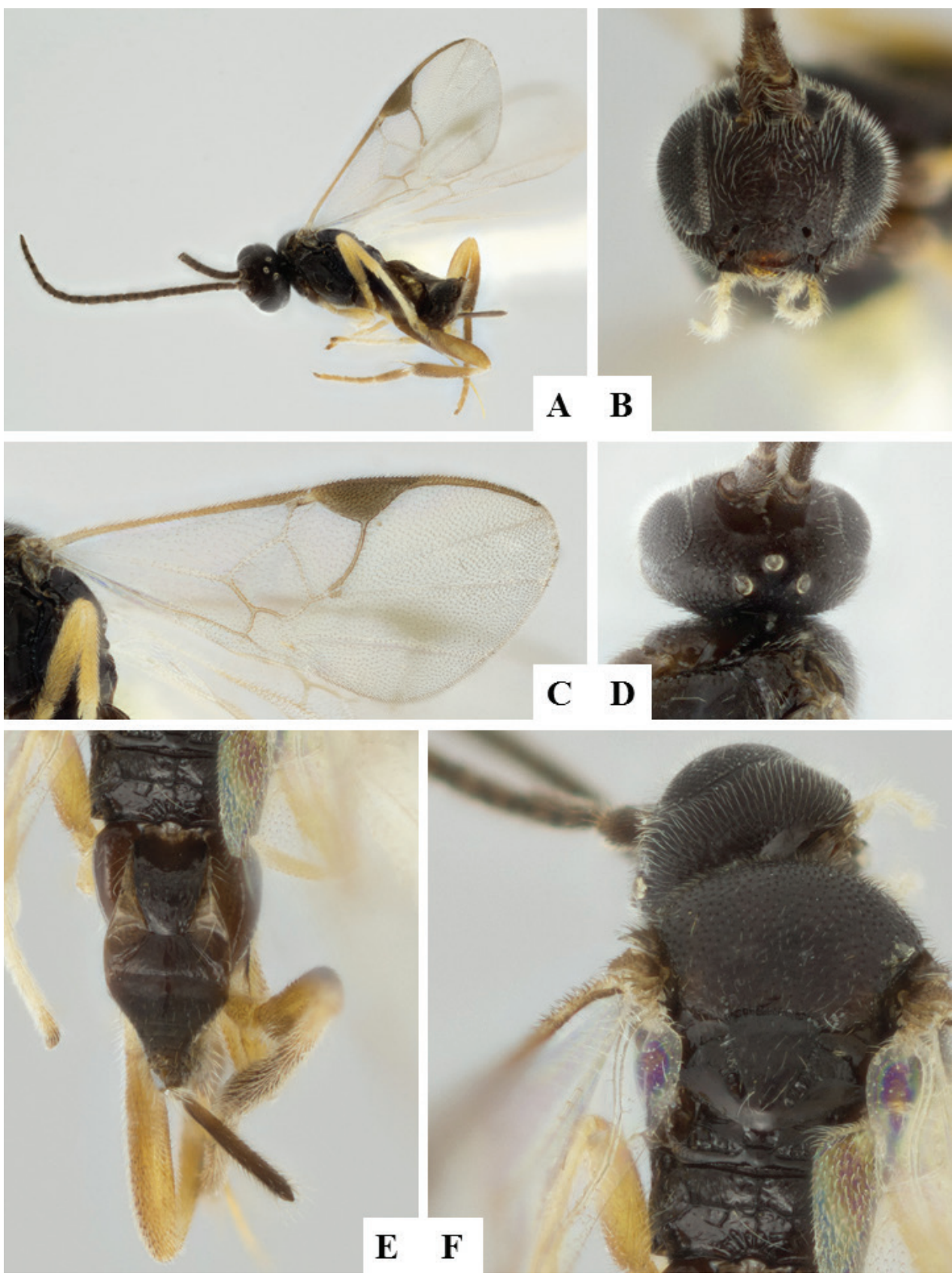

Figure 13. Clarkinella canadensis. A Habitus, lateral B Head, frontal C Wings D Head dorsal E Metasoma, dorsal F Head and mesosoma, dorsal. 

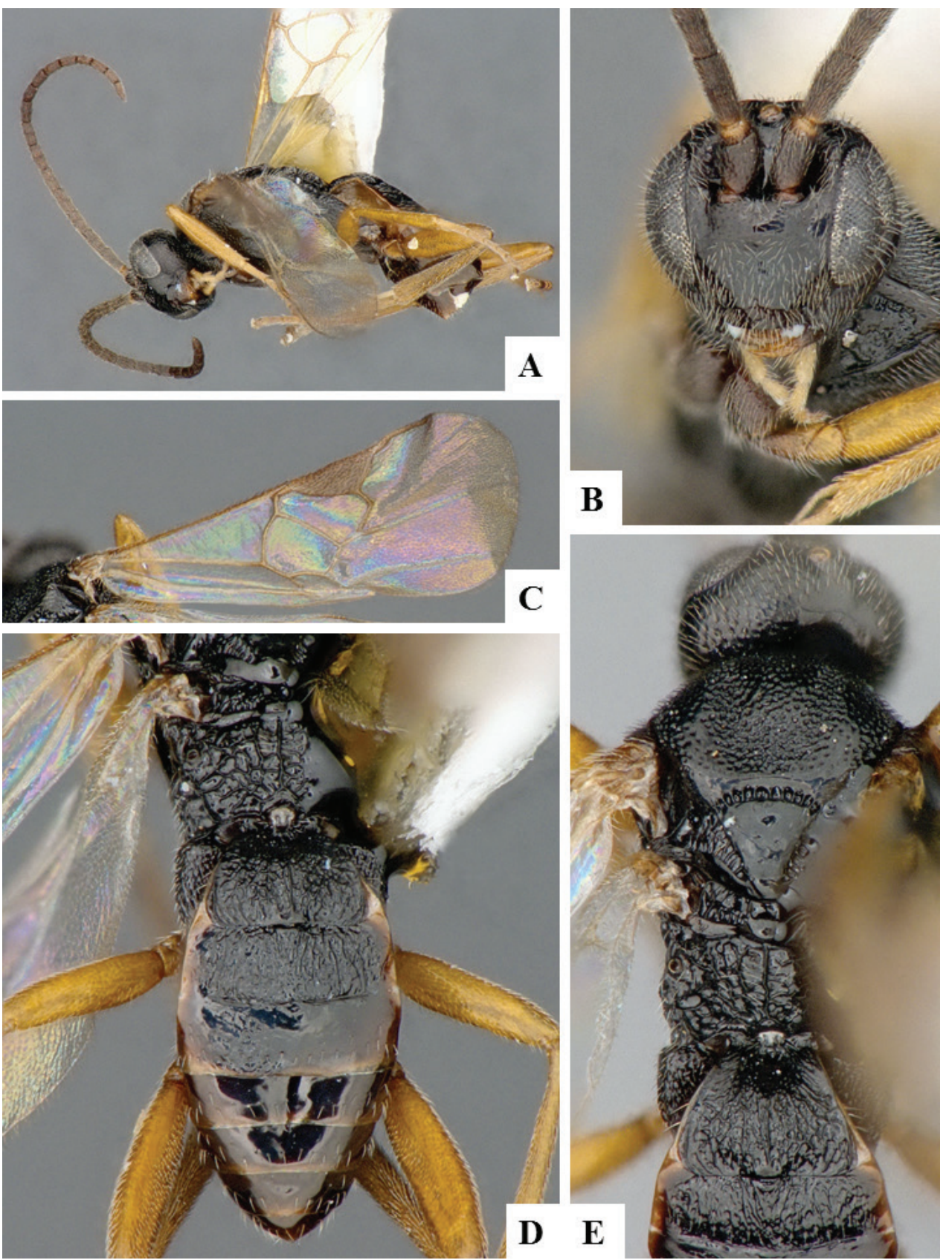

Figure 14. Cotesia acronyctae. A Habitus, lateral B Head, frontal C Wings D Metasoma, dorsal E Head and mesosoma, dorsal. 

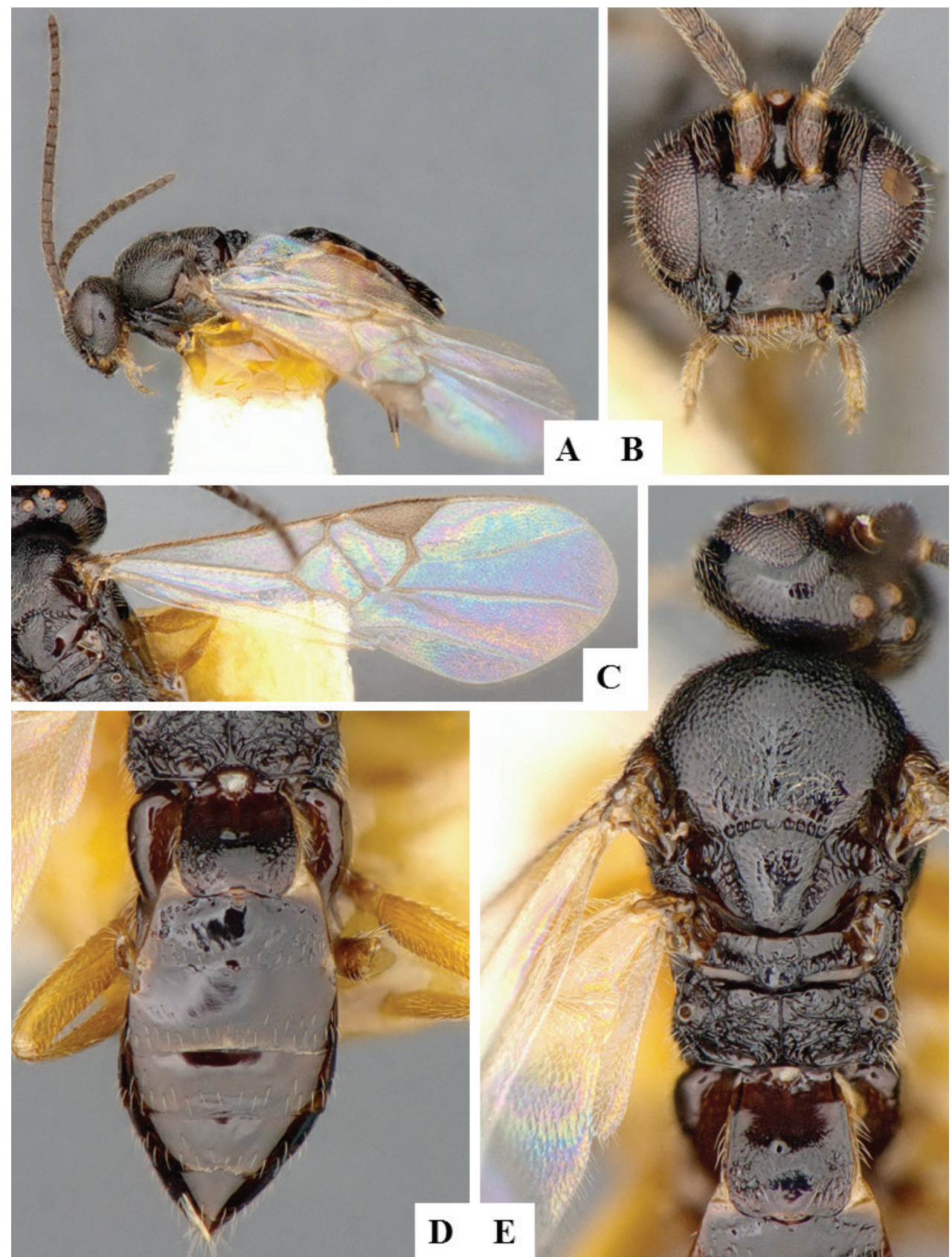

Figure 15. Cotesia atalantae. A Habitus, lateral B Head, frontal C Wings D Metasoma, dorsal E Head and mesosoma, dorsal. 
CNC474834, CNC474835, CNC474836, CNC474837, CNC474838, CNC474839, CNC474840, CNC474841, CNC474842, CNC474843, CNC474844, CNC474845, CNC474846, CNC474847, CNC474848, CNC474849, CNC474850, CNC474851, CNC474852, CNC474853, CNC474854, CNC474855, CNC474856, CNC474857, CNC474858, CNC474859, CNC474860, CNC474861, CNC474862, CNC474863, CNC474864, CNC474865, CNC474866, CNC474867, CNC474868, CNC474869, CNC474870, CNC474871, CNC474872, CNC474873, CNC474874; 45.194311 -75.838992, 27.vii.1973, J. E. H. Martin, Voucher Code: CNCHYM00361; Ottawa, 45.356083 -75.706933, 31.vii.1939, Hobbs, G.A., Voucher Code: CNC280772; 45.406631 -75.701407, 31.vii.1939, G.A. Hobbs, Voucher Code: CNC474800, CNC474801, CNC474802, CNC474803, CNC474804, CNC474805, CNC474806.

\section{Cotesia cerurae (Muesebeck, 1926)}

\section{Distribution. NEA.}

Material examined. Ontario, Stittsville, 45.258796 -75.92113, 21.i.1946, Voucher Code: CNCHYM00373; 23.i.1946, Voucher Code: CNCHYM00376; 24.i.1945, Voucher Code: CNCHYM00375; 25.i.1946, Voucher Code: CNCHYM00374; 45.259037 -75.920958, 24.i.1946, F.I.S , Voucher Code: CNC280776.

\section{Cotesia cingiliae (Muesebeck, 1931)}

\section{Distribution. NEA.}

Material examined. Ontario, Merivale, 45.340734 -75.727462, 7.ix.1948, Voucher Code: CNCHYM00381; 45.340750 -75.727475, 7.ix.1948, F.I.S., Voucher Code: CNC280779.

\section{Cotesia clisiocampae (Ashmead, 1903)}

\section{Distribution. NEA.}

Material examined. Ontario, Mer Bleue, Ottawa, 45.393578 -75.512128, 27.v.1927-27.v.1997, G.S. Walley, Voucher Code: CNC280781; 45.393585 -75.512138, 17.v.1927, G.S. Walley, Voucher Code: CNC474883, CNC474884, CNC474885, CNC474886, CNC474887, CNC474888, CNC474889, CNC474890, CNC474891, CNC474892, CNC474893, CNC474894; 21.v.1927, G.S. Walley, Voucher Code: CNC474875, CNC474876, CNC474877, CNC474878, CNC474879, CNC474880, CNC474881, CNC474882; 27.v.1927, G.S. Walley, Voucher Code: CNC474895, CNC474896; 45.393593 -75.512138, 27.v.1937, G. S. Walley, Voucher Code: CNCHYM00386. 


\section{Cotesia congregata (Say, 1836)}

Distribution. NEA, NEO.

Material examined. Ontario, Ottawa, city garden, $45.3561-75.707$, 1.ix.2007, H. Goulet, Voucher Code: CAM0268; Ottawa, 45.3825 -75.7137, 10.vii.1944, M. d'Aoust, Voucher Code: CNCHYM00396; 26.i.1942, F. I. Survey, Voucher Code: CNCHYM00394; 45.406631 -75.701407, 27.i.1942, F.I.S., Voucher Code: CNC474900; 30.i.1942, F.I.S., Voucher Code: CNC474899; 21.viii.1944, F.I.S., Voucher Code: CNC474897, CNC474898.

\section{Cotesia crambi (Weed, 1887)}

\section{Distribution. NEA.}

Material examined. Ontario, mixed forest near Manotick, Ottawa, 45.235 -75.624, 29.vi-16.vii.2007, A. Bennett, Voucher Code: CAM0542; Ottawa, city garden, 45.356 -75.707, 10.viii-1.ix.2007, H. Goulet, Voucher Code: CAM1034; 30.vii10.viii.2007, H. Goulet, Voucher Code: CAM0893.

\section{Cotesia depressa (Viereck, 1912)}

Distribution. NEA.

Material examined. Quebec, Gatineau Park, 45.600555-76.042647, 15.viii.1946, F.I.S., Voucher Code: CNCHYM00415.

Cotesia diacrisiae (Gahan, 1917)

Distribution. NEA.

Material examined. Quebec, Gatineau Park, 45.600556 -76.042647, 15.viii.1946, F.I.S., Voucher Code: CNC474901.

\section{Cotesia fiskei (Viereck, 1910)}

\section{Distribution. NEA.}

Material examined. Ontario, Stittsville, 45.258675 -75.921130, 1.viii.1951, F.I.S., Voucher Code: CNC474910, CNC474911, CNC474912, CNC474913, CNC474914, CNC474915, CNC474916, CNC474917, CNC474918, CNC474919, CNC474920, CNC474921; 31.vii.1951, F.I.S., Voucher Code: CNC474902, CNC474903, CNC474904, CNC474905, CNC474906, CNC474907, CNC474908, CNC474909; 45.258796 -75.92113, 31.vii.1951, Voucher Code: CNCHYM00466. 


\section{Cotesia flaviconchae (Riley, 1881)}

\section{Distribution. NEA.}

Material examined. Ontario, Ottawa, 45.356083 -75.706933, 15.x.1959, Martin, J.E.H., Voucher Code: CNC280802; 45.3825 -75.7137, 16.x.1959, J. E. H. Martin, Voucher Code: CNCHYM00470; 19.x.1959, J. E. H. Martin, Voucher Code: CNCHYM00468; 23.x.1959, J. E. H. Martin, Voucher Code: CNCHYM00469; 45.406631 -75.701407, 15.x.1959, J.E.H. Martin, Voucher Code: CNC474922, CNC474923, CNC474924, CNC474925, CNC474926; 16.x.1959, J.E.H. Martin, Voucher Code: CNC474927, CNC474928, CNC474929, CNC474930, CNC474931, CNC474932, CNC474933, CNC474934, CNC474935, CNC474936, CNC474937, CNC474938， CNC474939， CNC474940, CNC474941, CNC474942, CNC474943, CNC474944, CNC474945; 19.x.1959, J.E.H. Martin, Voucher Code: CNC474946, CNC474947, CNC474948; 23.x.1959, J.E.H. Martin, Voucher Code: CNC474949, CNC474950; 45.406633 -75.701408, x.1959, J.E.H. Martin, Voucher Code: CNC474951.

\section{Cotesia glomerata (Linnaeus, 1758)}

Distribution. Cosmopolitan.

Material examined. Ontario, Long Swamp, Ottawa, 45.3825 -75.7137, 7.ix.1943, E. G. lester, Voucher Code: CNCHYM00489; 45.419163 -75.709648, 7.ix.1963, Lester, E.G., Voucher Code: CNC280801.

\section{Cotesia halisidotae (Muesebeck, 1931)}

Distribution. NEA.

Material examined. Ontario, Ottawa, 45.406633 -75.701408, 1908, Voucher Code: CNC474952; 30.v.1908, Voucher Code: CNC474953.

\section{Cotesia hemileucae (Riley, 1881)}

Distribution. NEA.

Material examined. Ontario, Carlsbad Springs, 45.369133 -75.456226, 10.viii.1946, F.I.S., Voucher Code: CNC474958, CNC474959, CNC474960; 6.viii.1946, F.I.S., Voucher Code: CNC474954, CNC474955, CNC474956, CNC474957; 45.369194 -75.456140, 6.viii.1946, F.I.S., Voucher Code: CNC280807; 45.369254 -75.456097, 6.viii.1946, Voucher Code: CNCHYM00510. 


\section{Cotesia hyphantriae (Riley, 1887)}

Distribution. NEA, PAL.

Material examined. Ontario, Leitrum, 45.329824 -75.598377, 10.viii.1946, F.I.S., Voucher Code: CNC474968; 12.viii.1946, F.I.S., Voucher Code: CNC474964, CNC474965; 15.viii.1946, F.I.S., Voucher Code: CNC474966; 19.viii.1946, F.I.S., Voucher Code: CNC474967; 24.viii.1946, F.I.S., Voucher Code: CNC474962, CNC474963; Ottawa, 45.406631 -75.701407, 10.viii.1950, D.W. Peters, Voucher Code: CNC474961.

\section{Cotesia laeviceps (Ashmead, 1890)}

\section{Distribution. NEA.}

Material examined. Ontario, Ottawa, 45.3825 -75.7137, 25.v.1943, G. S. Walley, Voucher Code: CNCHYM00557; 45.406631 -75.701407, 25.v.1943, G.S. Walley, Voucher Code: CNC474969, CNC474970, CNC474971, CNC474972, CNC474973, CNC474974, CNC474975, CNC474976, CNC474977, CNC474978, CNC474979, CNC474980, CNC474981, CNC474982, CNC474983, CNC474984, CNC474985, CNC474986, CNC474987, CNC474988, CNC474989, CNC474990, CNC474991, CNC474992, CNC474993, CNC474994, CNC474995, CNC474996, CNC474997, CNC474998, CNC474999, CNC475000, CNC475001, CNC475002, CNC475003, CNC475004, CNC475005, CNC475006, CNC475007, CNC475008, CNC475009, CNC475010, CNC475011, CNC475012.

\section{Cotesia melanoscela (Ratzeburg, 1844)}

Fig. 16

Distribution. NEA. OTL, PAL.

Notes. This is mostly a Holarctic species, with some records from the Oriental region due to being a parasitoid of the Gypsy Moth, Lymantria dispar (Linnaeus, 1758).

Material examined. Ontario, Kilbirnie, 45.247853 -75.724400, 26.iii.1981, Voucher Code: CNC475048; 31.iii.1981, Voucher Code: CNC475046, CNC475047.

\section{Cotesia nemoriae (Ashmead, 1898)}

\section{Distribution. NEA.}

Material examined. Ontario, Ottawa, 45.406631 -75.701407, 14.vi.1943, Voucher Code: CNC475049; Quebec, Old Chelsea, 45.503548 -75.797963, 25.v.1942, Voucher Code: CNCHYM00605; 45.541317 -75.867939, 25.v.1942, F.I.S., Voucher Code: CNC475050. 

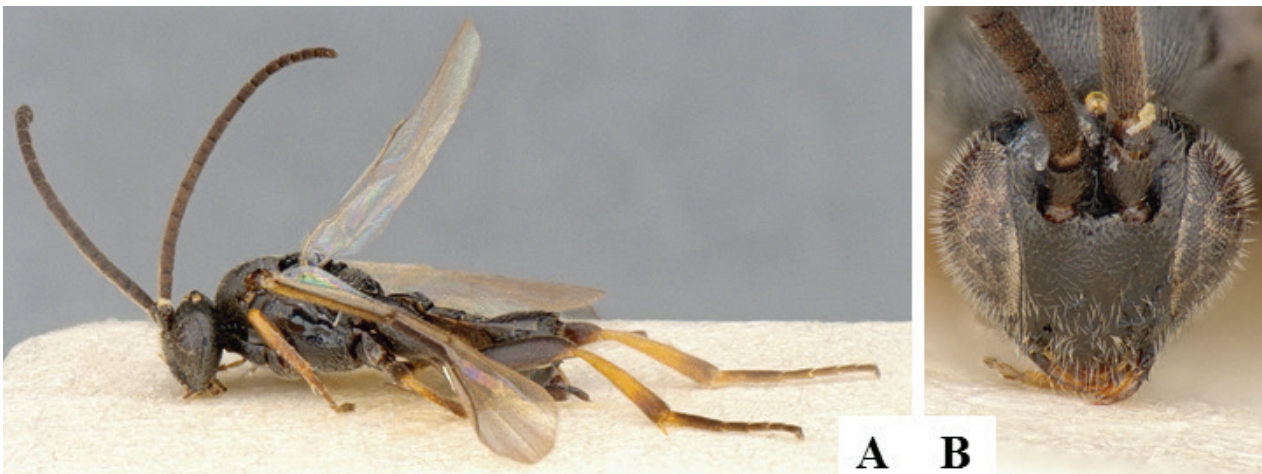

A B
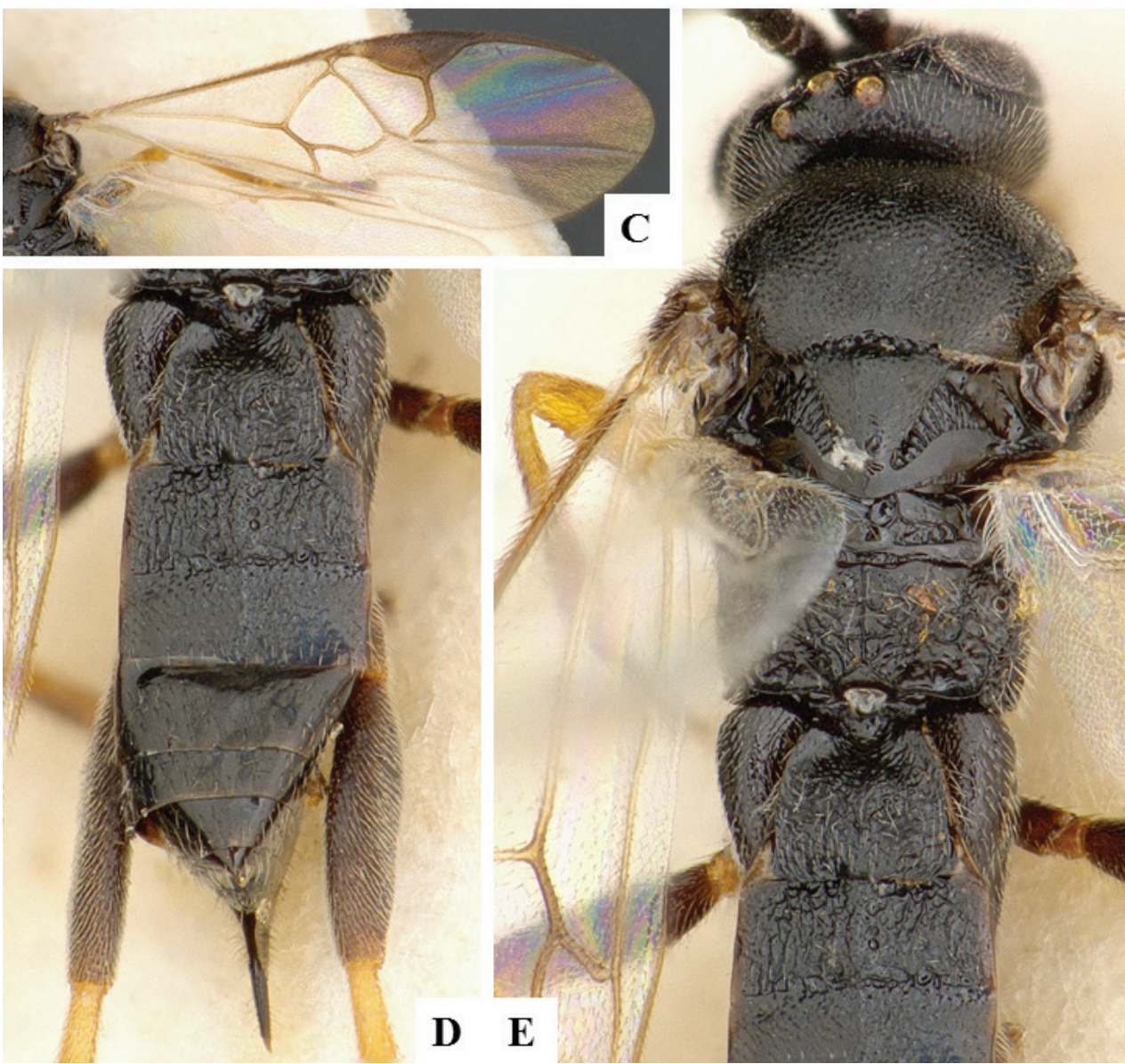

Figure 16. Cotesia melanoscela. A Habitus, lateral B Head, frontal C Wings D Metasoma, dorsal E Head and mesosoma, dorsal. 


\section{Cotesia parastichtidis (Muesebeck, 1921)}

Distribution. NEA.

Material examined. Ontario, Ottawa, city garden, $45.356-75.707$, H. Goulet, Voucher Code: CAM1038; 45.356083 -75.706933, malaise trap, 8-16.vi.2007, Goulet, H. , Voucher Code: CNC280831; Ottawa, 45.3825 -75.7137, 1.iv.1941, Voucher Code: CNCHYM00636; 45.406633 -75.701408, 1.vi.1941, F.I.S., Voucher Code: CNC475054, CNC475055; 31.v.1941, F.I.S., Voucher Code: CNC475052, CNC475053.

\section{Cotesia phobetri (Rohwer, 1915)}

Distribution. NEA.

Material examined. Ontario, Ottawa, $45.406631-75.701407,23 . i i .1948$, F.I.S., Voucher Code: CNC475056; 3.ix.1947, F.I.S., Voucher Code: CNC475059, CNC475060.

\section{Cotesia schizurae Ashmead, 1898}

\section{Distribution. NEA.}

Material examined. Ontario, Ottawa, 45.356083 -75.706933, 6.viii.1959, Downes, A.J., Voucher Code: CNC280849; 45.3825 -75.7137, 6.viii.1959, A. J. Downes, Voucher Code: CNCHYM00699; 45.406631 -75.701407, 6.viii.1959, A.J. Downes, Voucher Code: CNC475061, CNC475062, CNC475063, CNC475064, CNC475065.

\section{Cotesia xylina (Say, 1836)}

Distribution. NEA.

Material examined. Ontario, Ottawa, city garden, $45.356-75.707$, 23.vii.2009, L. Masner, Voucher Code: CNCH0464; Quebec, Gatineau Park, 45.60057 -76.042647, 24.v.2007, L. Masner, Voucher Code: HYM00001130.

\section{Cotesia jft02}

Distribution. NEA.

Notes. This species corresponds in BOLD to BIN BOLD:ABY4229, with all specimens collected in North America.

Material examined. Ontario, mixed forest, 45.235 -75.624, 7-19.vi.2007, A. Bennett, Voucher Code: CAM0538; Ottawa, city garden, 45.356 -75.707, 10.viii-1. 
ix.2007, H. Goulet, Voucher Code: CAM1033; 20-29.ix.2008, H. Goulet, Voucher Code: CAM1036; 26.vi-13.vii.2007, H. Goulet, Voucher Code: HYM00000999.

\section{Cotesia jft03}

\section{Distribution. NEA.}

Notes. This undescribed species, so far only recorded from Canada seems to be an important parasitoid (at least locally) of the Diamondback Moth, Plutella xylostella (Linnaeus, 1758). This species corresponds in BOLD to BIN BOLD:AAA9378.

Material examined. Ontario, North Gower to Smith Falls, $1 \mathrm{~km} \mathrm{~N}$ of Rd 6 \& Montague Bdy Rd, 45.033 -75.9, 15.vi.2004, Bennett \& Barnes, Voucher Code: HYM00001204, HYM00001205, HYM00001206; Ottawa, CNC breeding program, Ottawa, 45.3825 -75.7137, 31.iii.2008, Jose L. Fernandez Triana, Voucher Code: CPWH-0033, СРWH-0034, СРWH-0035, CPWH-0036, СРWH-0037, СРWH0038, СРWH-0039, СРWH-0040, СРWH-0041, СРWH-0042, СРWH-0043, CPWH-0044, СРWH-0045, СРWH-0046, СРWH-0047, СРWH-0048, СРWH0049, CPWH-0050, CPWH-0051, CPWH-0052; Ottawa, city garden, 45.3561 -75.7069, 30.vii-10.viii.2007, H. Goulet, Voucher Code: CNCH0510; 45.356100 -75.706900, 30.vii-10.viii.2007, H. Goulet, Voucher Code: CNCH0509, CNCH0511.

\section{Cotesia jft07}

Distribution. NEA, PAL.

Notes. This species seems to be related to the European Cotesia salebrosa (Marshall, 1885), or perhaps it is that same species (in which case it would be the first record of the species for the Nearctic). Pending further study of more material from other localities, we prefer to keep it as an undescribed for the time being. This species corresponds in BOLD to BIN BOLD:ABZ3751.

Material examined. Ontario, North Gower to Smith Falls, $1 \mathrm{~km} \mathrm{~N}$ of Rd 6 \& Montague Bdy Rd, 45.033 -75.9, 15.vi.2004, Bennett \& Barnes, Voucher Code: HYM00001132, HYM00001133, HYM00001135, HYM00001136, HYM00001137, HYM00001138, HYM00001139, HYM00001140, HYM00001141, HYM00001142, HYM00001143, HYM00001144, HYM00001145; Ottawa, city garden, 45.356 -75.707, 19.ix-8.xi.2007, H. Goulet, Voucher Code: HYM00000997; Quebec, Chelsea, near old visitor center, 45.541075 -75.867938, 11.xi.2008, J. Fernandez, Voucher Code: CAM0569, CAM0570.

\section{Cotesia jft09}

Distribution. NEA, PAL. 
Notes. This species seems to be related to a complex of species, from both Europe and North America. For the time being is left as an undescribed species, until more studies of the Holarctic fauna are carried out. This species corresponds in BOLD to BIN BOLD:AAA6099.

Material examined. Ontario, $1.6 \mathrm{~km} \mathrm{SW}$ of Manion Corners, Carleton Co., 45.25 -76.083, 1.iv.2004, R. Layberry, Voucher Code: CNCH0950; 25.iii.2004, R. Layberry, Voucher Code: CNCH0951.

\section{Cotesia jft20}

\section{Distribution. NEA.}

Notes. This species corresponds in BOLD to BIN BOLD:AAD6124, with all specimens collected in southern Ontario.

Material examined. Ontario, Ottawa, city garden, 45.3561 -75.7069, 8-16. vi.2007, H. Goulet, Voucher Code: CAM1037.

\section{Cotesia jft22}

\section{Distribution. NEA.}

Notes. This species corresponds in BOLD to BIN BOLD:AAF0583, with all specimens collected in North America.

Material examined. Ontario, Ottawa, city garden, 45.3561 -75.7069, 8-16. vi.2007, H. Goulet, Voucher Code: CAM1039.

\section{Cotesia jft42}

Distribution. NEA.

Notes. This species corresponds in BOLD to BIN BOLD:AAR9059, with only a single specimen known from Ottawa.

Material examined. Ontario, mixed forest, 45.235 -75.624, 29.vi-16.vii.2007, A. Bennett, Voucher Code: CAM0540.

\section{Cotesia jft44}

\section{Distribution. NEA.}

Notes. This species corresponds in BOLD to BIN BOLD:AAR9061, with only a single specimen known from Ottawa.

Material examined. Ontario, mixed forest, 45.235 -75.624, 29.vi-16.vii.2007, A. Bennett, Voucher Code: CAM0541. 


\section{Cotesia jft53}

Distribution. NEA.

Notes. This species corresponds in BOLD to BIN BOLD:ABX6366, with all specimens collected in North America.

Material examined. Ontario, $5 \mathrm{~km}$ NW of Almonte, Hwy 49, Burnt Land, Alvar Prov. Park, Almonte, 45.255 -76.14, 29.v.2008, Goulet \& Fernandez, Voucher Code: CAM0332, CAM0333.

\section{Cotesia Whitfield26}

Distribution. NEA.

Notes. This species corresponds in BOLD to BIN BOLD:AAH2149, with all specimens collected in southern Ontario.

Material examined. Ontario, mixed forest, 45.234700 -75.624000, 29.vi-16. vii.2007, A. Bennett, Voucher Code: CAM0539; Ottawa, city garden, 45.356100 -75.706900, 16-26.vi.2007, H. Goulet, Voucher Code: CAM0897.

\section{Diolcogaster auripes (Provancher, 1886)}

\section{Distribution. NEA.}

Material examined. Ontario, Aylmer West, 45.4 -75.85, 16.viii.1972, Voucher Code: CNCHYM00790; Innisville, 45.055468 -76.250497, 14.viii.1963, W.R.M. Mason, Voucher Code: CNC475090, CNC475091; mixed forest, 45.2347 -75.624, 29.vi-16. vii.2007, A. Bennett, Voucher Code: CAM0546; Ottawa , 45.419164 -75.709650, 11.vii.1951, Guppy, J.C., Voucher Code: CNC280871; Ottawa, 45.3825 -75.7137, 11.vii.1957, J. C. Guppy, Voucher Code: CNCHYM00787; 45.406631 -75.701407, 28.vi.1947, W.R.M. Mason, Voucher Code: CNC475087; Stittsville, 45.258675 -75.921130, 5.vi.1963, W.R.M. Mason, Voucher Code: CNC475089; 45.258796 -75.92113, 14.vi.1969, W. R. M. Mason, Voucher Code: CNCHYM00789; Thurso, 45.596990 -75.243571, 20.viii.1958, L.A. Kelton, Voucher Code: CNC475088; Quebec, Hull, 45.428309 -75.713353, 13.vi.1965, Voucher Code: CNCHYM00788; 45.428550 -75.714554, Malaise trap, vi.1965, Voucher Code: CNC475092; Old Chelsea, 45.500055 -75.814616, 26.v.1964, J.R. Vockeroth, Voucher Code: CNC475093; Thurso, 45.59729 -75.243828, 20.viii.1985, L. A. Kelton, Voucher Code: CNCHYM00786.

\section{Diolcogaster claritibia (Papp, 1959)}

Fig. 17

Distribution. NEA, PAL. 

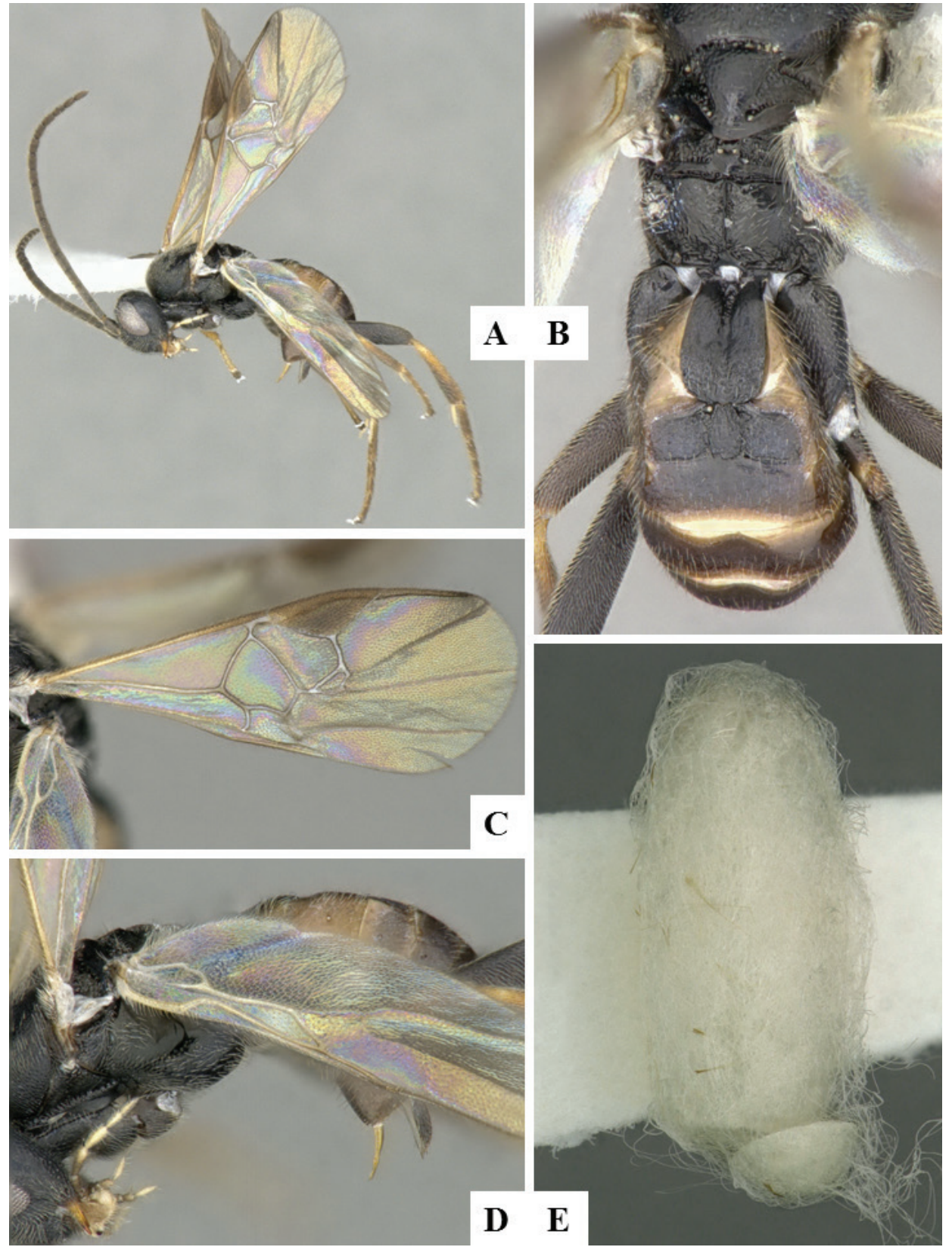

Figure 17. Diolcogaster claritibia. A Habitus, lateral B Mesosoma (partially) and metasoma dorsal C Wings D Mesosoma (partially) and metasoma, lateral E Cocoon.

Material examined. Ontario, CEF, DBM Field Cage Trials, 45.389959 -75.711949, 23.vi.2010, P. Mason, S. Girardoz, Voucher Code: CNCHYM01690, CNCHYM01691, CNCHYM01692, CNCHYM01693, CNCHYM01694, CNCHYM01695. 


\section{Diolcogaster facetosa (Weed, 1888)}

\section{Distribution. NEA.}

Material examined. Ontario, Innisville, 45.054942 -76.250619, 29.viii.1963, W. R. M. Mason, Voucher Code: CNCHYM00822; 45.055468 -76.250497, 28.vii.1963, W.R.M. Mason, Voucher Code: CNC475104; 31.v.1963, W.R.M. Mason, Voucher Code: CNC475103; Ottawa, 45.406631 -75.701407, 15.vii.1987, W.R.M. Mason, Voucher Code: CNC475099; 20.vii.1955, W.R.M. Mason, Voucher Code: CNC475105, CNC475106, CNC475107; 26.vii.1955, W.R.M. Mason, Voucher Code: CNC475108, CNC475109, CNC475110, CNC475111, CNC475112, CNC475113, CNC475114, CNC475115, CNC475116; Stittsville, 45.258675 -75.921130, 22.vii.1963, W.R.M. Mason, Voucher Code: CNC475102; 27.v.1963, W.R.M. Mason, Voucher Code: CNC475100; 5.vi.1963, W.R.M. Mason, Voucher Code: CNC475101; Woodlawn, 45.375 -76.083, 6.viii.2008, L. Masner, Voucher Code: MIC000622; Quebec, Aylmer West, 45.395345 -75.844876, Malaise trap, 1.vii.1973, Voucher Code: CNC475118; 6-13.vii.1973, Voucher Code: CNC475117; Aylmer, 45.395345 -75.844876, 10.vi.1924, C.H. Curran, Voucher Code: CNC475095; 27.vii.1924, C.H. Curran, Voucher Code: CNC475097; 29.v.1924, C.H. Curran, Voucher Code: CNC475094; 8.vi.1924, C.H. Curran, Voucher Code: CNC475096; Old Chelsea, 45.541315 -75.867938, 13.v.1965, G.S. Walley, Voucher Code: CNC475119; Wakefield, 45.631572 -75.924033, 16.iv.1938, F.I.S., Voucher Code: CNC475120.

\section{Diolcogaster jft30}

\section{Distribution. NEA.}

Notes. This species corresponds in BOLD to BIN BOLD:AAB0185, with all specimens collected in North America.

Material examined. Ontario, $5 \mathrm{~km}$ NW of Almonte, Hwy 49, Burnt Land, Alvar Prov. Park, Almonte, 45.2549 -76.14, 29.v.2008, Goulet \& Fernandez, Voucher Code: CAM0366, CAM0367.

\section{Diolcogaster jft35}

\section{Distribution. NEA.}

Notes. This species corresponds in BOLD to BIN BOLD:AAX9631, with only a single specimen known from Ottawa.

Material examined. Ontario, mixed forest, 45.2347 -75.624, 19-29.vi.2007, A. Bennett, Voucher Code: CAM0545. 


\section{Diolcogaster jft38}

\section{Distribution. NEA.}

Notes. This species corresponds in BOLD to BIN BOLD:AAI6267, with only a single specimen known from Ottawa.

Material examined. Quebec, Gatineau Park, 45.60057 -76.042647, 24.v.2007, L. Masner, Voucher Code: HYM00001284.

\section{Diolcogaster jft41}

\section{Distribution. NEA.}

Notes. This species corresponds in BOLD to BIN BOLD:AAB0192, with all specimens collected in Canada.

Material examined. Ontario, mixed forest, 45.2347 -75.624, 29.vi-16.vii.2007, A. Bennett, Voucher Code: CAM0547, CAM0548, CAM0549.

\section{Distatrix carolinae Fernandez-Triana, 2010}

Fig. 18

Distribution. NEA.

Notes. The status of this species as a potential member of the Species Candidate Lists of COSEWIC was assessed by Fernandez Triana (2014).

Material examined. Quebec, Summit King Mt. Old Chelsea, 45.500147 -75.814703, 26.vi.1977, M. Sandborne, Voucher Code: CNC23940.

\section{Dolichogenidea absona (Muesebeck, 1965)}

Fig. 19

Distribution. NEA.

Material examined. Ontario, Mer Bleue, 45.393593 -75.512138, 26.ii.1961, Freeman \& Lewis, Voucher Code: MIC000241, MIC000242.

\section{Dolichogenidea cacoeciae (Riley, 1881)}

Fig. 20

\section{Distribution. NEA.}

Material examined. Ontario, Merivale, 45.325948 -75.719082, 22.vi.1935, E.G. Lester, Voucher Code: CNC475188, CNC475189, CNC475190, CNC475191; Ottawa, 45.356083 -75.706933, 9.iv.1953, C.D. Miller, Voucher Code: CNC280909; 

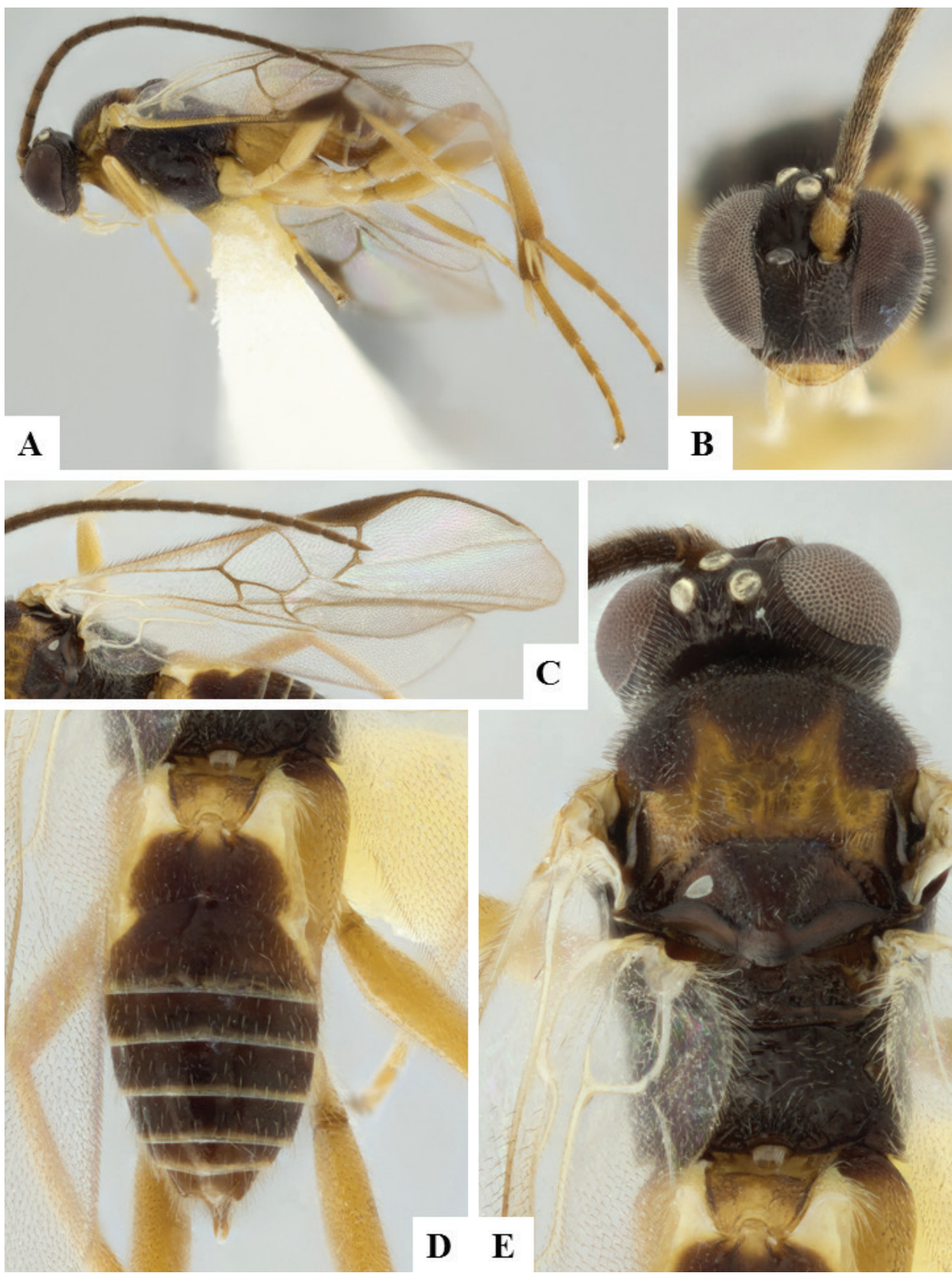

Figure 18. Distatrix carolinae. A Habitus, lateral B Head, frontal C Wings D Metasoma, dorsal E Head and mesosoma, dorsal. 


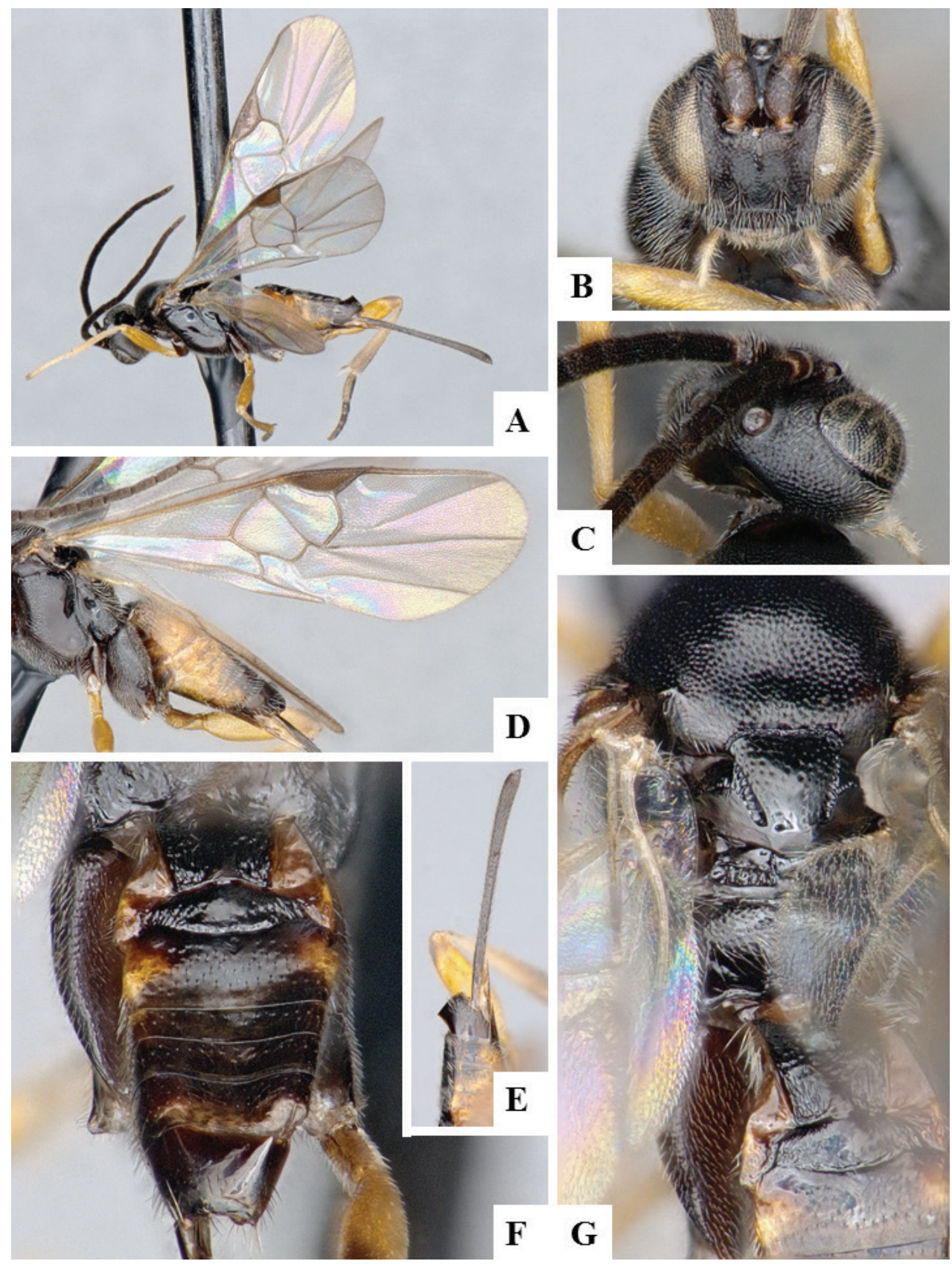

Figure 19. Dolichogenidea absona. A Habitus, lateral B Head, frontal C Head, frontal D Wings E Ovipositor sheaths $\mathbf{F}$ Metasoma, dorsal G Mesosoma, dorsal. 

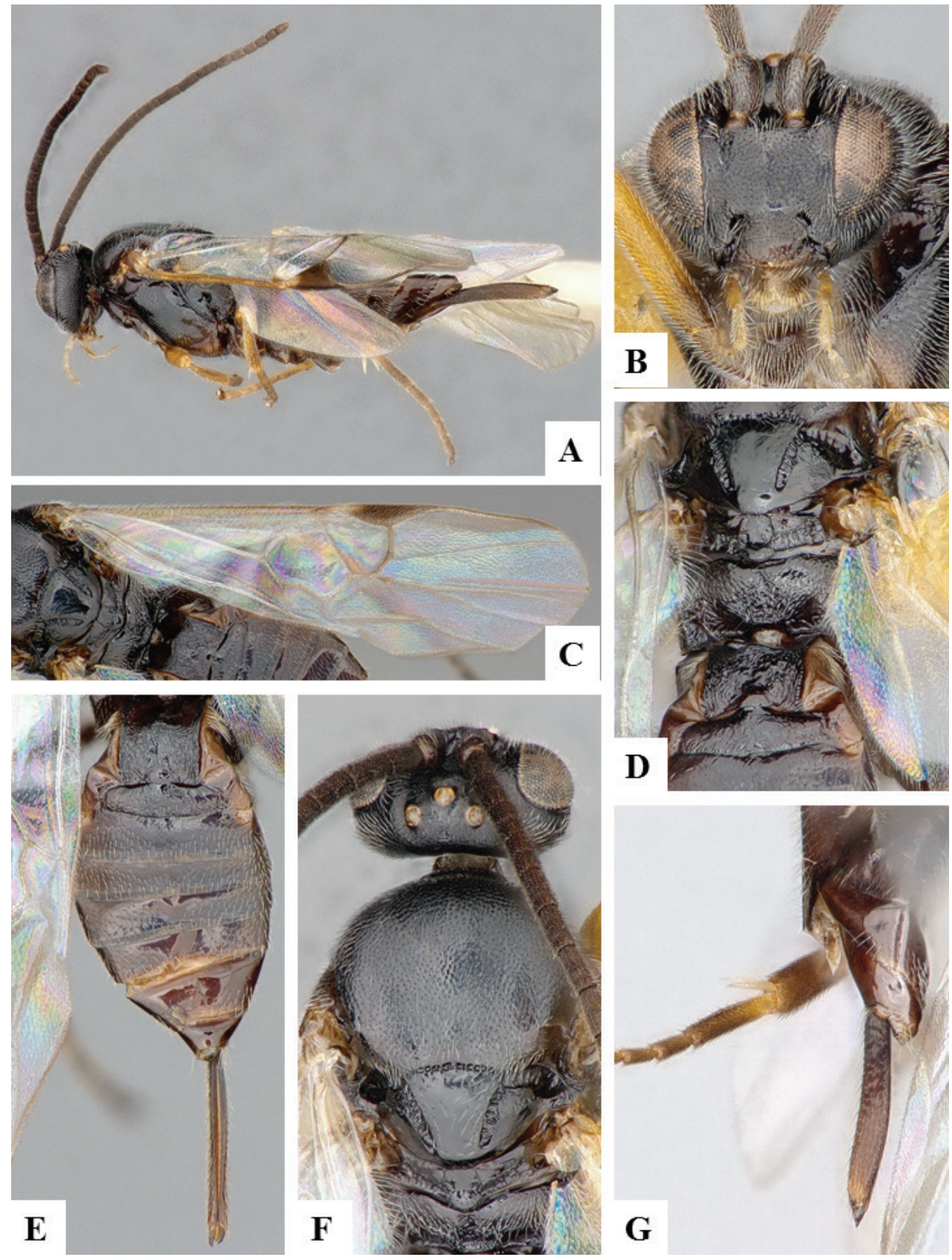

Figure 20. Dolichogenidea cacoeciae. A Habitus, lateral B Head, frontal C Wings D Mesosoma and metasoma (partially), dorsal E Metasoma, dorsal F Head and mesosoma, dorsal G Ovipositor sheaths. 
45.3825-75.7137, 14.xi.1969, A. Sauve, Voucher Code: CNCHYM00994; 45.406631 -75.701407, 12.v.1969, A. Sauve, Voucher Code: CNC475161, CNC475162; 13.xi.1969, A. Sauve, Voucher Code: CNC475142, CNC475143, CNC475144, CNC475145, CNC475146, CNC475147, CNC475148, CNC475149, CNC475150; 14.xi.1969, A. Sauve, Voucher Code: CNC475130, CNC475131, CNC475132, CNC475133, CNC475134, CNC475135, CNC475136, CNC475137, CNC475138, CNC475139, CNC475140, CNC475141; 19.ii.1969, A. Sauve, Voucher Code: CNC475154, CNC475155, CNC475156, CNC475157, CNC475158, CNC475159, CNC475160; 20.ii.1969, A. Sauve, Voucher Code: CNC475151, CNC475152, CNC475153; 21.ii.1969, A. Sauve, Voucher Code: CNC475163; 5.v.1944, G.S. Walley, Voucher Code: CNC475193, CNC475194, CNC475195; 6.v.1944, G.S. Walley, Voucher Code: CNC475196, CNC475197, CNC475198, CNC475199, CNC475200, CNC475201; 9.iv.1953, C.D. Miller, Voucher Code: CNC475121, CNC475122, CNC475123, CNC475124, CNC475125, CNC475126, CNC475127, CNC475128, CNC475129; Quebec, Hull, 45.428550 -75.714554, 2.v.1965, G.S.Walley, Voucher Code: CNC475192.

\section{Dolichogenidea paralechiae (Muesebeck, 1932)}

Fig. 21

Distribution. NEA.

Material examined. Ontario, Kemptville, 45.016409 -75.646449, 9.v.1952, Voucher Code: CNC475203, CNC475204; Stittsville, 45.258675 -75.921130, 23.vi.1951, F.I.S., Voucher Code: CNC475202.

\section{Dolichogenidea renaulti (Mason, 1974)}

Fig. 22

\section{Distribution. NEA.}

Material examined. Ontario, South March, 45.337746 -75.957675, 1.vi.1964, C.D. Miller, Voucher Code: CNC475214; 10.vi.1963, C.D. Miller, Voucher Code: CNC280950; 10.vi.1964, C.D. Miller, Voucher Code: CNC475211; 16.v.1963, C.D. Miller, Voucher Code: CNC475205, CNC475206, CNC475207; 16.vi.1964, C.D. Miller, Voucher Code: CNC475212; 17.v.1963, C.D. Miller, Voucher Code: CNC475208, CNC475209; 19.vi.1961, C.D. Miller, Voucher Code: CNC475216; 23.vi.1961, C.D. Miller, Voucher Code: CNC475215; 24.vi.1963, C.D. Miller, Voucher Code: CNC475210; 28.vi.1961, C.D. Miller, Voucher Code: CNC475217, CNC475218; 4.vi.1964, C.D. Miller, Voucher Code: CNC475213; 45.348507 -75.923123, 29.v.1961, C. Miller, Voucher Code: MIC000262; 45.348508 -75.923125, 16.v.1963, C. Miller, Voucher Code: MIC000256; 27.v.1965, C. Miller, Voucher Code: MIC000257. 

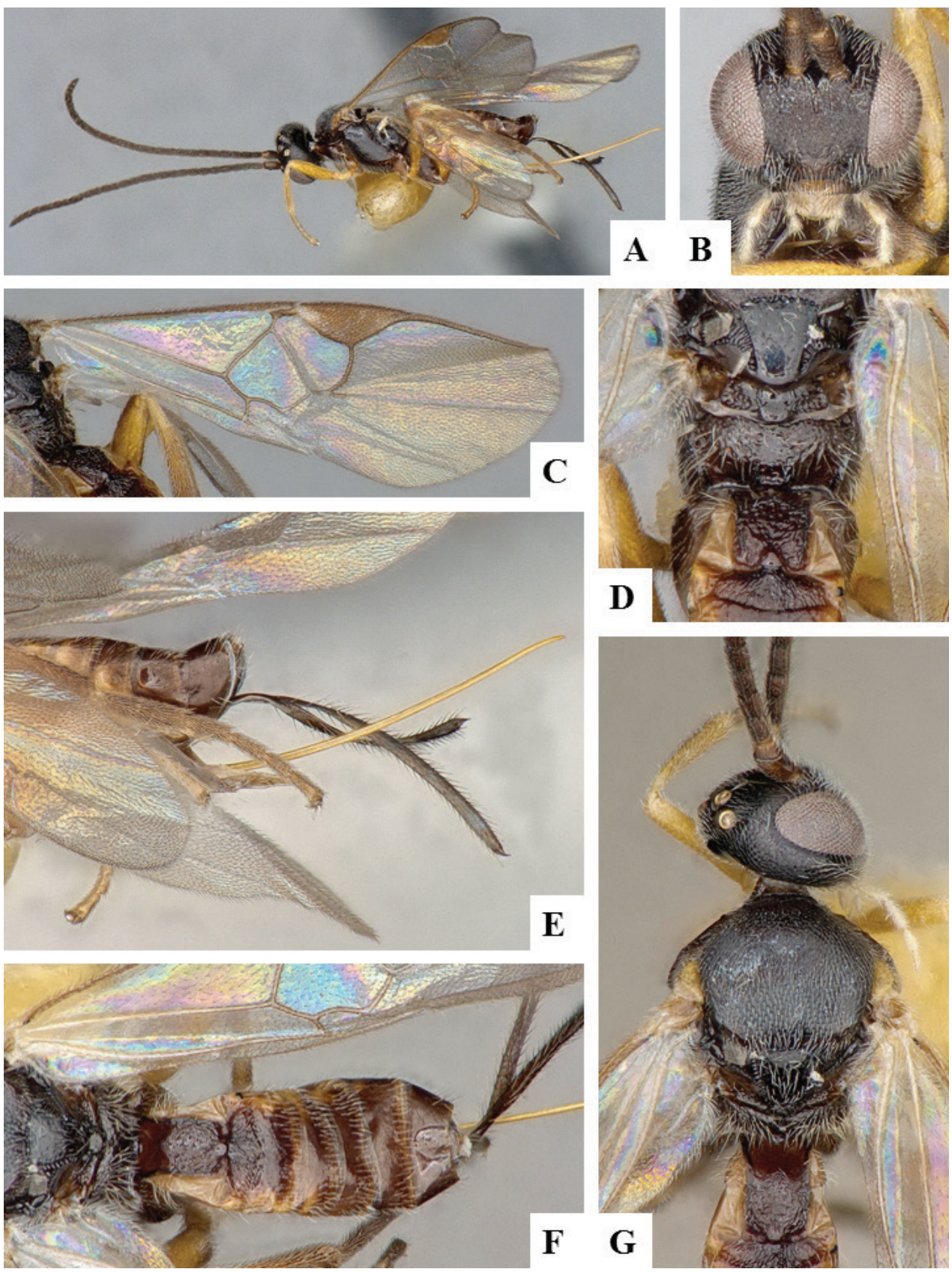

Figure 2I. Dolichogenidea paralechiae. A Habitus, lateral B Head, frontal C Wings D Mesosoma and metasoma (partially), dorsal E Metasoma, lateral F Metasoma, dorsal E Head and mesosoma, dorsal. 

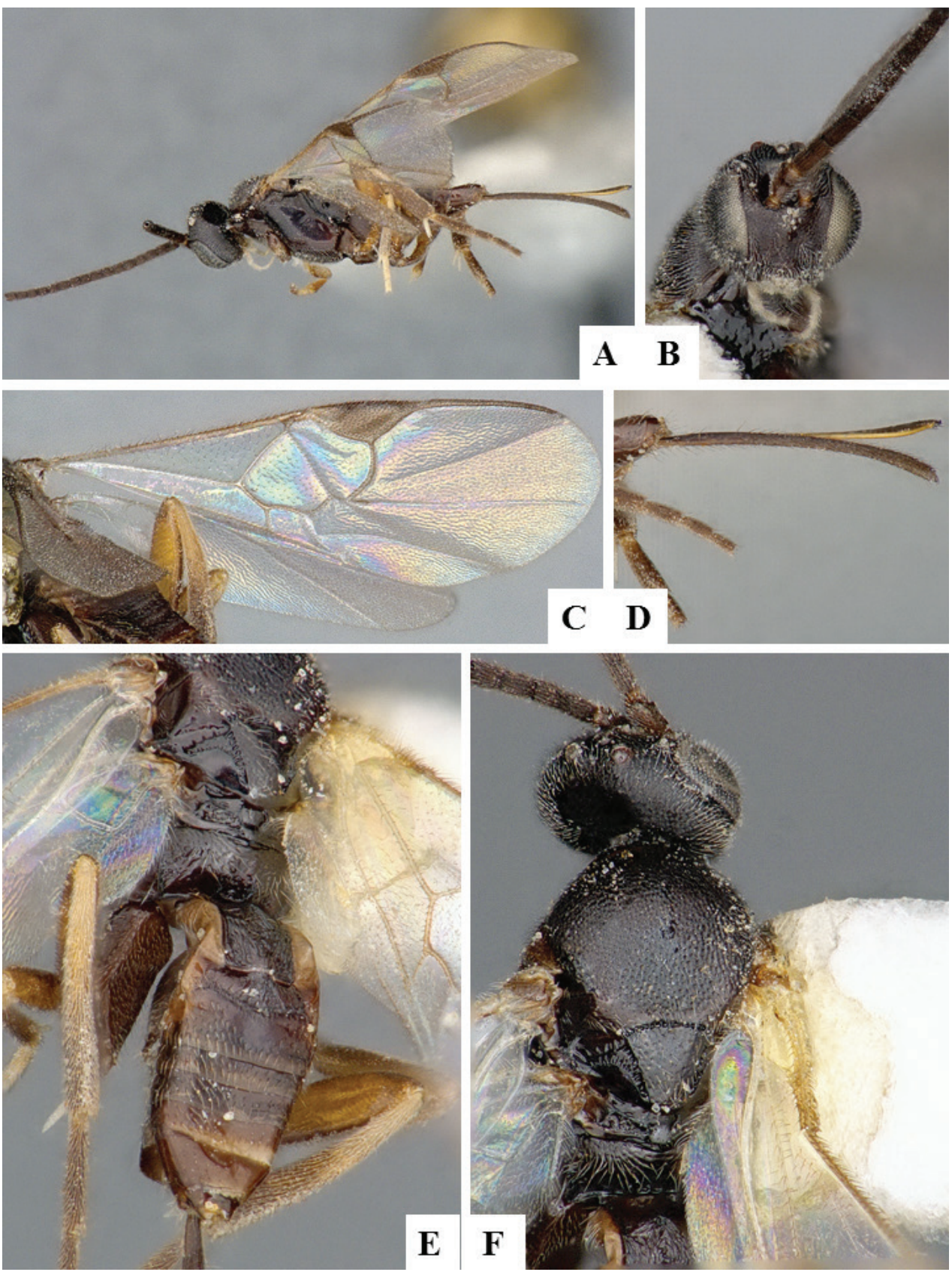

Figure 22. Dolichogenidea renaulti. A Habitus, lateral B Head, frontal C Wings D Ovipositor sheaths E Mesosoma (partially) and metasoma, dorsal F Head and mesosoma, dorsal. 
Dolichogenidea solenobiae (Walley, 1935)

Fig. 23

\section{Distribution. NEA.}

Material examined. Ontario, Ottawa, 45.3825 -75.7137, 31.iii.1945, G. S. Walley, Voucher Code: CNCHYM01141.

\section{Dolichogenidea thujae (Muesebeck, 1935)}

Fig. 24

\section{Distribution. NEA.}

Material examined. Ontario, Fallowfield, 45.267410 -75.829929, 18.vii.1960, C.D. Miller, Voucher Code: CNC475222; 19.vii.1960, C.D. Miller, Voucher Code: CNCHYM01157; 45.267531 -75.829886, 28.vii.1960, C.D. Miller, Voucher Code: CNC280957; Lanark, 45.018287 -76.365297, 27.vi.1951, Voucher Code: CNCHYM01159; 45.018354 -76.365257, 26.vi.1951, F.I.S., Voucher Code: CNC475224; 27.vi.1951, F.I.S., Voucher Code: CNC475223; Quebec, Aylmer, 45.395345 -75.844876, 14.vii.1960, C.D. Miller, Voucher Code: CNC475220; 15.vii.1960, C.D. Miller, Voucher Code: CNC475219; 18.vii.1960, C.D. Miller, Voucher Code: CNC475221; 45.4 -75.85, 19.vii.1960, Voucher Code: CNCHYM01158.

\section{Dolichogenidea jft02}

Distribution. NEA.

Notes. This species corresponds in BOLD to BIN BOLD:AAA4312, with all specimens collected in North America.

Material examined. Ontario, mixed forest, 45.2347 -75.624, 7-19.vi.2007, A. Bennett, Voucher Code: CAM0534.

\section{Dolichogenidea jft12}

Distribution. NEA.

Notes. This species corresponds in BOLD to BIN BOLD:AAC9784, with all specimens collected in eastern Canada.

Material examined. Ontario, Ottawa, city garden, $45.3561-75.707$, 1.ix.2007, H. Goulet, Voucher Code: CAM0044, CAM0087, CAM0149; Woodlawn, 45.375 -76.083, 8.ix.2008, L. Masner, Voucher Code: MIC000606. 


\section{Dolichogenidea jft13}

Distribution. NEA.

Notes. This species corresponds in BOLD to BIN BOLD:AAC0737, with all specimens collected in Canada.

Material examined. Ontario, Ottawa, city garden, 45.3561 -75.707, 13.vii.2007, H. Goulet, Voucher Code: CAM0057; 23.vii.2007, H. Goulet, Voucher Code: CAM0048.

\section{Dolichogenidea jft14}

Distribution. NEA.

Notes. This species corresponds in BOLD to BIN BOLD:AAA5986, with all specimens collected in Canada.

Material examined. Ontario, Ottawa, city garden, 45.3561 -75.7069, 16.vii.2009, L. Masner, Voucher Code: CNCH1020.

\section{Dolichogenidea jft18}

Distribution. NEA.

Notes. This species corresponds in BOLD to BIN BOLD:AAH2146, with all specimens collected in eastern Canada.

Material examined. Ontario, Ottawa, city garden, 45.3561 -75.707, 13.vii.2007, H. Goulet, Voucher Code: CAM0060.

\section{Glyptapanteles militaris (Walsh, 1861)}

Distribution. NEA, NEO, PAL.

Material examined. Ontario, Ottawa, City Garden, 45.381015 -75.715397, Malaise trap, 10.viii-1.ix.2007, H. Goulet, Voucher Code: CNC482042, CNC482043, CNC482044, CNC482045, CNC482046, CNC482047, CNC482048, CNC482049, CNC482050, CNC482051， CNC482052, CNC482053, CNC482054, CNC482055, CNC482056, CNC482057, CNC482058, CNC482059, CNC482060, CNC482061, CNC482062, CNC482063, CNC482064, CNC482065, CNC482066, CNC482067, CNC482068; 23-30.vii.2007, H. Goulet, Voucher Code: CNC482079, CNC482080, CNC482081, CNC482082, CNC482083, CNC482084, CNC482085, CNC482086, CNC482087, CNC482088, CNC482089, CNC482090, CNC482091, CNC482092, CNC482093, CNC482094, CNC482095, CNC482096, CNC482097, CNC482098, CNC482099, CNC482100, CNC482101, CNC482102, CNC482103, CNC482104, CNC482105, CNC482106, 

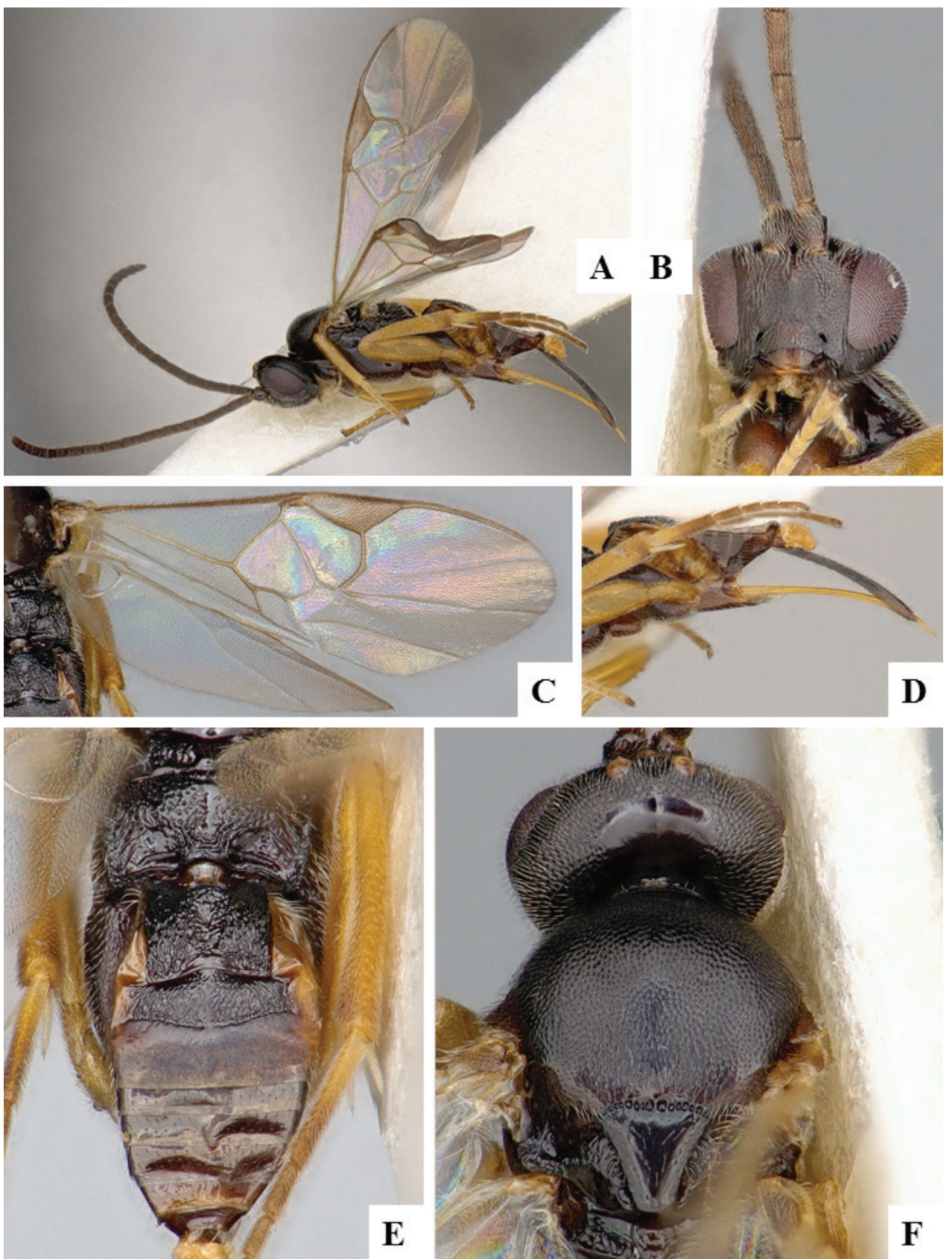

Figure 23. Dolichogenidea solenobiae. A Habitus, lateral B Head, frontal C Wings D Ovipositor sheaths and ovipositor E Metasoma, dorsal F Head and mesosoma, dorsal. 

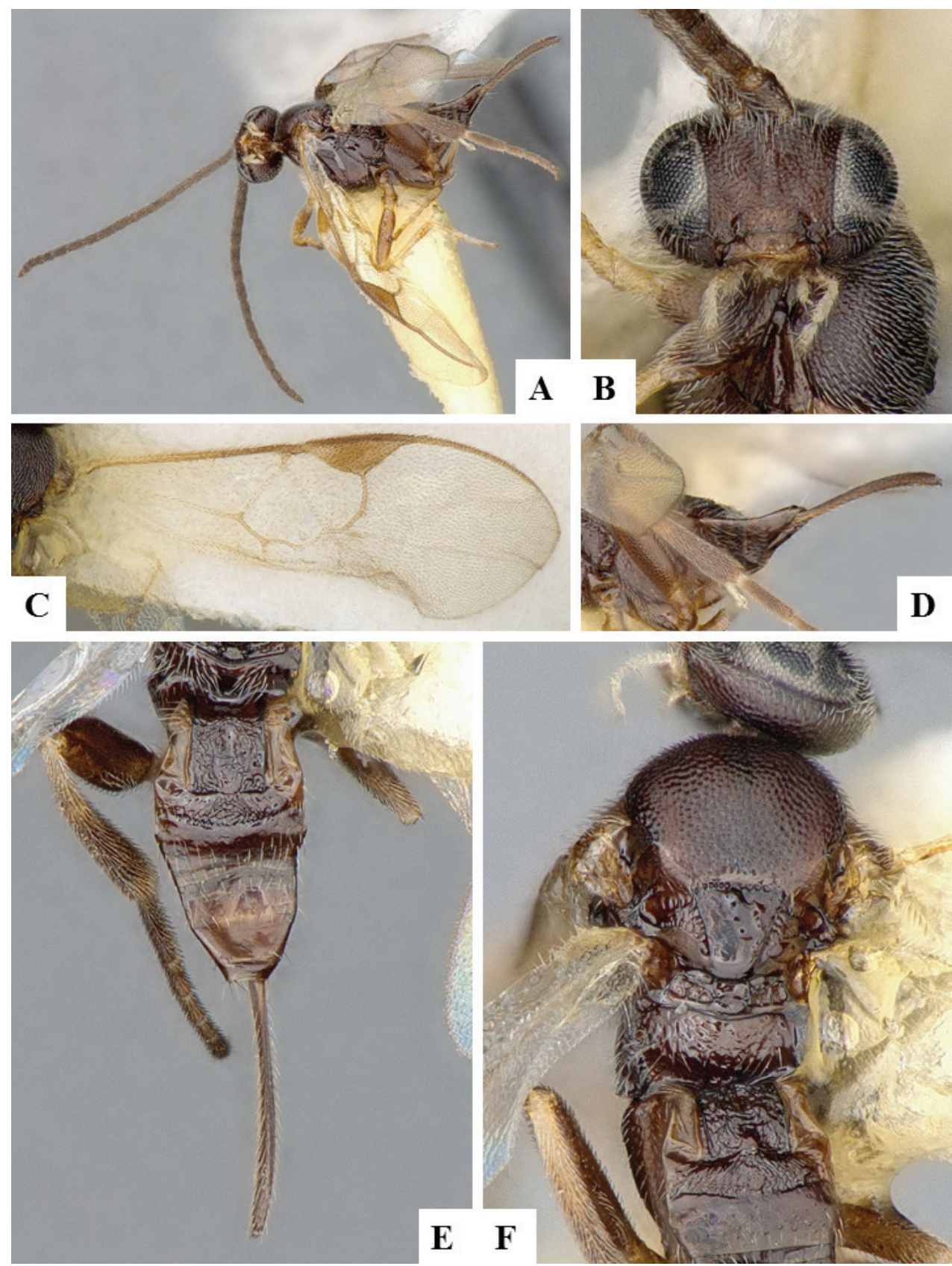

Figure 24. Dolichogenidea thujae. A Habitus, lateral B Head, frontal C Wings D Ovipositor sheaths and ovipositor $\mathbf{E}$ Metasoma, dorsal $\mathbf{F}$ Head and mesosoma, dorsal. 
CNC482107, CNC482108, CNC482109, CNC482110, CNC482111, CNC482112, CNC482113, CNC482114, CNC482115, CNC482116, CNC482117, CNC482118, CNC482119, CNC482120, CNC482121, CNC482122, CNC482123, CNC482124, CNC482125, CNC482126, CNC482127, CNC482128, CNC482129, CNC482130, CNC482131, CNC482132, CNC482133, CNC482134; 24-30.v.2007, H. Goulet, Voucher Code: CNC482073, CNC482074, CNC482075, CNC482076; 26.vi-13. vii.2007, H. Goulet, Voucher Code: CNC482078; 30.vii-10.viii.2007, H. Goulet, H.Goulet, Voucher Code: CNC481889, CNC481890, CNC481891, CNC481928, CNC481929, CNC481930, CNC481931, CNC481932, CNC481933, CNC481934, CNC481935, CNC481936, CNC481937, CNC481938, CNC481939, CNC481940, CNC481941, CNC481942, CNC481943, CNC481944, CNC481945, CNC481946, CNC481947, CNC481948, CNC481949, CNC481950, CNC481951, CNC481952, CNC481953, CNC481954, CNC481955, CNC481956, CNC481957, CNC481958, CNC481959, CNC481960, CNC481961, CNC481962, CNC481963, CNC481964, CNC481965, CNC481966, CNC481967, CNC481968, CNC481969, CNC481970, CNC481971, CNC481972, CNC481973, CNC481974, CNC481975, CNC481976, CNC481977, CNC481978, CNC481979, CNC481980, CNC481981, CNC481982, CNC481983, CNC481984, CNC481985, CNC481986, CNC481987, CNC481988, CNC481997, CNC481998, CNC481999, CNC482000, CNC482001, CNC482002, CNC482003, CNC482004, CNC482005, CNC482006, CNC482007, CNC482008, CNC482009, CNC482010, CNC482011, CNC482012, CNC482013, CNC482014, CNC482015, CNC482016, CNC482017, CNC482018, CNC482019, CNC482020, CNC482021, CNC482022, CNC482023, CNC482024, CNC482025, CNC482026, CNC482027, CNC482028, CNC482029, CNC482030, CNC482031, CNC482032, CNC482033, CNC482034, CNC482035, CNC482036, CNC482037, CNC482038, CNC482039, CNC482040, CNC482041, CNC481878, CNC481879, CNC481880, CNC481881, CNC481882, CNC481883, CNC481884, CNC481885, CNC481886, CNC481887, CNC481888; 8-16.vi.2007, H. Goulet, Voucher Code: CNC482077; Ottawa, city garden, 45.381015 -75.715397, Malaise trap, 1-19.ix.2007, H. Goulet, Voucher Code: CNC475269, CNC475270, CNC475271, CNC475272; 10.viii1.ix.2007, H. Goulet, Voucher Code: CNC481892, CNC481893, CNC481894, CNC481895, CNC481896, CNC481897, CNC481898, CNC481899, CNC481900, CNC481901, CNC481902, CNC481903, CNC481904, CNC481905, CNC481906, CNC481907, CNC481908, CNC481909, CNC481910, CNC481911, CNC481912, CNC481913, CNC481914, CNC481915, CNC481916, CNC481917, CNC481918, CNC481919, CNC481920, CNC481921, CNC481922, CNC481923, CNC481924, CNC481925, CNC481926, CNC481927; 23-30.vii.2007, H. Goulet, Voucher Code: CNC475244, CNC475245, CNC475246, CNC475247, CNC475248, CNC475249, CNC475250, CNC475251, CNC475252, CNC475253, CNC475254, CNC475255, CNC475256, CNC475257, CNC475258, CNC475259, CNC475260, CNC475261, CNC475262, CNC475263, CNC475264, CNC475265; 2430.v.2007, H. Goulet, Voucher Code: CNC481852, CNC481853, CNC481854; 26.vi-13.vii.2007, H. Goulet, Voucher Code: CNC481855; 30.vii-10.viii.2007, H. 
Goulet, Voucher Code: CNC475238, CNC475239, CNC475240, CNC475241, CNC475242, CNC475243, CNC475273; 8-16.vi.2007, H. Goulet, Voucher Code: CNC475266, CNC475267, CNC475268; Ottawa, 45.381015 -75.715397, Malaise trap, 10.viii-1.ix.2007, H. Goulet, Voucher Code: CNC481856, CNC481857, CNC481858, CNC481859, CNC481860, CNC481861, CNC481862, CNC481863, CNC481864, CNC481865, CNC481866, CNC481867, CNC481868, CNC481869, CNC481870, CNC481871, CNC481872, CNC481873, CNC481874, CNC481875, CNC481876, CNC481877; 21.vii.1957, Guppy, J.C., J. C. Guppy, J.C. Guppy, Voucher Code: CNC280985, CNCHYM01317, CNC475225, CNC475226, CNC475227, CNC475228, CNC475229, CNC475230, CNC475231, CNC475232, CNC475233, CNC475234, CNC475235, CNC475236, CNC475237.

\section{Glyptapanteles pallipes (Reinhard, 1880)}

Fig. 25

Distribution. NEA, PAL.

Material examined. Ontario, Ottawa, 45.406631 -75.701407, 30.ix.1925, D. D. Gray, -, Voucher Code: CNC482135, CNC482136, CNC482137, CNC482138, CNC482139, CNC482140, CNC482141, CNC482142, CNC482143, CNC482144, CNC482145, CNC482146, CNC482147, CNC482148, CNC482149, CNC482150, CNC482161, CNC482162, CNC482163, CNC482164, CNC482165, CNC482166, CNC482167, CNC482168, CNC482169, CNC482170, CNC482171, CNC482172, CNC482173, CNC482174, CNC482175, CNC482176, CNC482177, CNC482178, CNC482179, CNC482180, CNC482181, CNC482182, CNC482183, CNC482184, CNC482185, CNC482186, CNC482187, CNC482188, CNC482189, CNC482190, CNC482191, CNC482192, CNC482193, CNC482194, CNC482195, CNC482196, CNC482197, CNC482198, CNC482199, CNC482200, CNC482201, CNC482202, CNC482203, CNC482204, CNC482205, CNC482206, CNC482207, CNC482208, CNC482209, CNC482210, CNC482211, CNC482212, CNC482213, CNC482214, CNC482215, CNC482216, CNC482217, CNC482218, CNC482219, CNC482220, CNC482151; Quebec, Cantley, Gatineau Co., 45.507888 -75.761995, 22.vii.1973, A.B. Cruins, M. B. Guins, Voucher Code: CNCHYM01322, CNC482152, CNC482153, CNC482154, CNC482155, CNC482156, CNC482157, CNC482158, CNC482159, CNC482160.

\section{Glyptapanteles sp.}

Distribution. NEA.

Notes. This species corresponds in BOLD to BIN BOLD:AAA4784, with all specimens collected in North America. 
Material examined. Ontario, mixed forest, 45.2347 -75.624, 19-29.vi.2007, A. Bennett, Voucher Code: CAM0554; 7-19.vi.2007, A. Bennett, Voucher Code: CAM0555, CAM0556, CAM0557, CAM0558; Ottawa, city garden, 45.3561 -75.707, 30.vii.2007, H. Goulet, Voucher Code: CAM0264, CAM0265, CAM0274; Woodlawn, 45.375 -76.083, 17.viii.2008, L. Masner, Voucher Code: MIC000613; 6.viii.2008, L. Masner, Voucher Code: MIC000607, MIC000608, MIC000609, MIC000610, MIC000611, MIC000612; 8.ix.2008, L. Masner, Voucher Code: MIC000614, MIC000615, MIC000616, MIC000617

\section{Hygroplitis melligaster (Provancher, 1886)}

Fig. 26

\section{Distribution. NEA.}

Material examined. Ontario, $2 \mathrm{~km} \mathrm{SW}$ of Innisville, $45.054942-76.250619$, 26.vi.1991, Sharkey \& Read, Voucher Code: GOU0314; Aylmer West, 45.395345 -75.844876, Malaise trap, 1-3.viii.1972, Voucher Code: CNC482223; 20-24.vii.1972, Voucher Code: CNC482221, CNC482222; 45.4 -75.85, 22.vii.1972, Voucher Code: CNCHYM01376; Bells Corners, 45.322247 -75.833249, 26.vii.1939, O. Peck, Voucher Code: CNC482224, CNC482225; Galetta, 45.4333392 -76.250086, 22.vii.1942, G. S. Walley, Voucher Code: CNC482226; Innisville, 45.055468 -76.250497, 12.vii.1963, W. R. M. Mason, Voucher Code: CNC482228; 16.vii.1963, W. R. M. Mason, Voucher Code: CNC482229; 18.vii.1963, W. R. M. Mason, Voucher Code: CNC482230, CNC482231, CNC482232; 22.vii.1963, W. R. M. Mason, Voucher Code: CNC482233, CNC482234, CNC482235, CNC482236, CNC482237, CNC482238, CNC482239, CNC482240, CNC482248; 25.vii.1963, W. R. M. Mason, Voucher Code: CNC482241, CNC482242, CNC482243, CNC482244; 28.vii.1963, W. R. M. Mason, Voucher Code: CNC482245, CNC482246, CNC482247; Kemptville, 45.016409 -75.64602, 28.vii.1983, W. Mason, Voucher Code: CNCHYM01375; 45.016409 -75.646449, Maple Forest, Malaise trap, 19-26.vii.1983, W. Mason, Voucher Code: CNC482259, CNC482260; 26-31.vii.1983, W. Mason, Voucher Code: CNC482261, CNC482262; Mer Bleue, Ottawa, 45.393594 -75.512139, 9.vii.1982, L. Dumouchel, Voucher Code: CNCHYM01378; Ottawa, 45.406631 -75.701407, Voucher Code: CNC482227; Stittsville, 45.258675 -75.921130, 18.vii.1963, W. R. M. Mason, Voucher Code: CNC482257; 2.vii.1963, W. R. M. Mason, Voucher Code: CNC482249; 22.vii.1963, W. R. M. Mason, Voucher Code: CNC482250; 25.vii.1963, W. R. M. Mason, Voucher Code: CNC482251, CNC482252; 28.vii.1963, W. R. M. Mason, Voucher Code: CNC482253; 9.viii.1963, W. R. M. Mason, Voucher Code: CNC482254, CNC482255, CNC482256; 45.258796 -75.92113, 22.viii.1963, W. R. M. Mason, Voucher Code: CNCHYM01364; 45.259037 -75.920958, 22.viii.1963, W. R. M. Mason, Voucher Code: CNC280997. 


\section{Hypomicrogaster ecdytolophae (Muesebeck, 1922)}

\section{Distribution. NEA, NEO.}

Notes. Valerio and Whitfield (2015) synonymized this species under H. zonaria. However, based on the specimens and associated data that we have been able to see (e.g., DNA and host records), H. zonaria (sensu Valerio and Whitfield 2015) seems to actually comprise several, distinct species. Pending future work on the New World fauna of Hypomicrogaster, we are considering in this paper $H$. ecdytolophae as separate from $H$. zonaria.

Material examined. Ontario, Merivale, 45.340734 -75.727462, 19.vii.1943, G. S. Walley, Voucher Code: CNCHYM01386; 45.340749 -75.727473, 19.vii.1943, G.S. Walley, Voucher Code: CNC280999; Ottawa, city garden, 45.356100 -75.707000, 19.x.2007, H. Goulet, Voucher Code: CAM0260; Ottawa, 45.406631 -75.701407, 8.iii.1939, C. H. Young, Voucher Code: CNC482258.

\section{Hypomicrogaster zonaria (Say, 1836)}

\section{Distribution. NEA.}

Notes. In this paper we are considering this species as separate from $H$. ecdytolophae (see comments above).

Material examined. Ontario, Aylmer West, 45.395345 -75.844876, Malaise trap, 13-17.vii.1972, Voucher Code: CNC482280; 17-20.vii.1972, Voucher Code: CNC482281; 27-31.vii.1972, Voucher Code: CNC482279; 3-8.viii.1972, Voucher Code: CNC482278; Flint Hill nr. Kemptville, 45.016346-75.646535, 16-23.viii.1983, Mason, Voucher Code: CNC482271, CNC482272, CNC482273, CNC482274, CNC482275, CNC482276, CNC482277; Kemptville, 45.016409 -75.64602, 30.viii.1983, W. Mason, Voucher Code: CNCHYM01441; 45.016409 -75.646449, Maple Forest, Malaise trap, 1-6.ix.1983, W. Mason, Voucher Code: CNC482301, CNC482302, CNC482303, CNC482304; 19-26.vii.1983, W. Mason, Voucher Code: CNC482294, CNC482295, CNC482296; 26-31.vii.1983, W. Mason, Voucher Code: CNC482291, CNC482292, CNC482293; Maple Forest, -, Malaise trap, 29.vii.1983, W. Mason, Voucher Code: CNC482305, CNC482306, CNC482289, CNC482290; Maple Forest, Malaise trap, 30.vii.1983, W. Mason, Voucher Code: CNC482285, CNC482286, CNC482287, CNC482288; 6-13.ix.1983, W. Mason, Voucher Code: CNC482297, CNC482298, CNC482299, CNC482300; 9-16. vii.1983, W. Mason, Voucher Code: CNC482307, CNC482308, CNC482309, CNC482310, CNC482311, CNC482312, CNC482313, CNC482314, CNC482315, CNC482316, CNC482317, CNC482318, CNC482319, CNC482320, CNC482321, CNC482322, CNC482323, CNC482324, CNC482325, CNC482326; Nr. Kemptville, 45.016409 -75.646449, Sunny dec. for., 16-23.viii.1983, Dumouchel \& Perkins, Voucher Code: CNC482282, CNC482283, CNC482284; Stittsville, Carleton Co., 

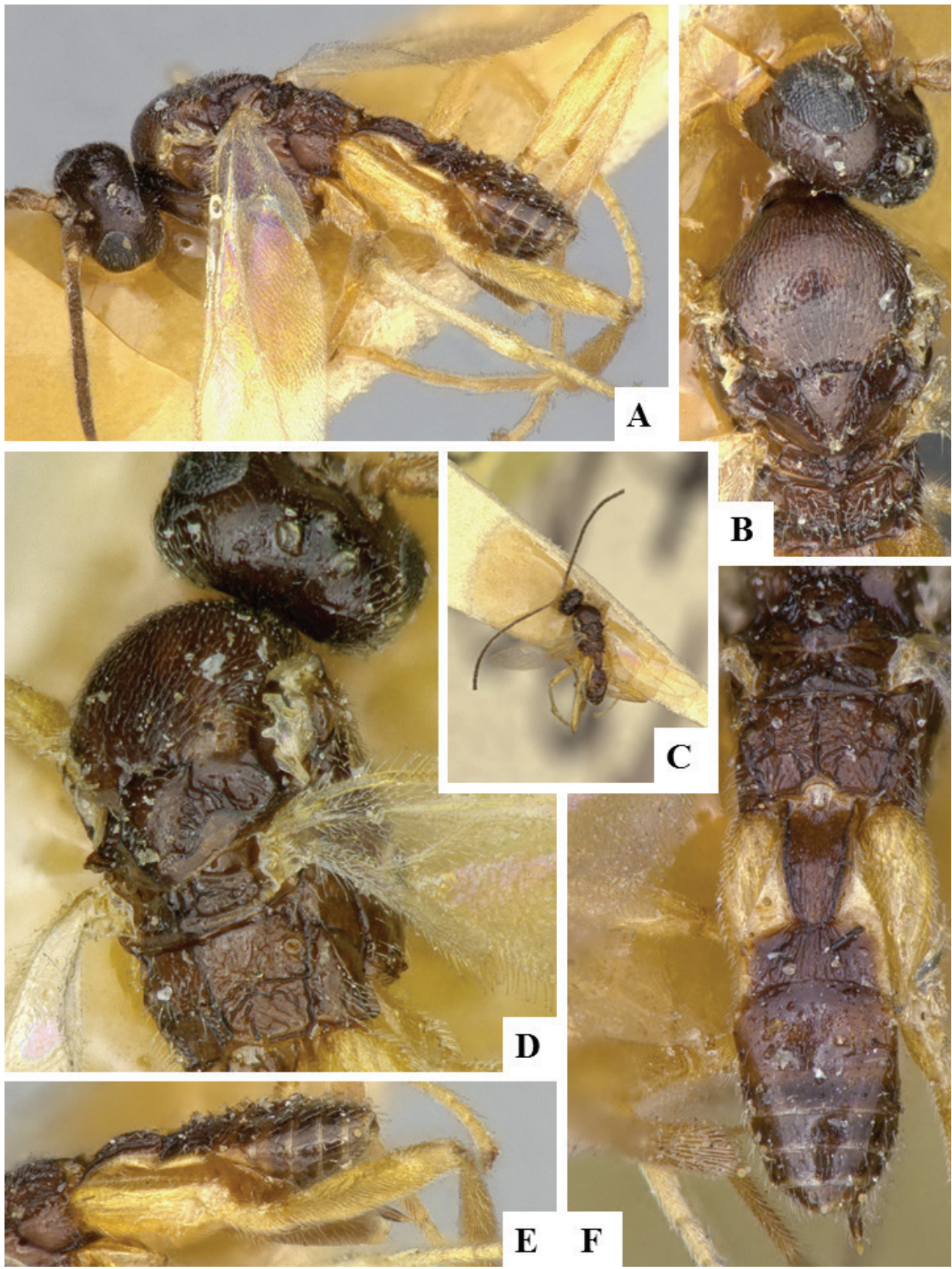

Figure 25. Glyptapanteles pallipes. A Habitus, lateral B Head and mesosoma, dorsal C Habitus, dorsal D Head and mesosoma, dorso-laterally E Metasoma, lateral F Metasoma, dorsal. 

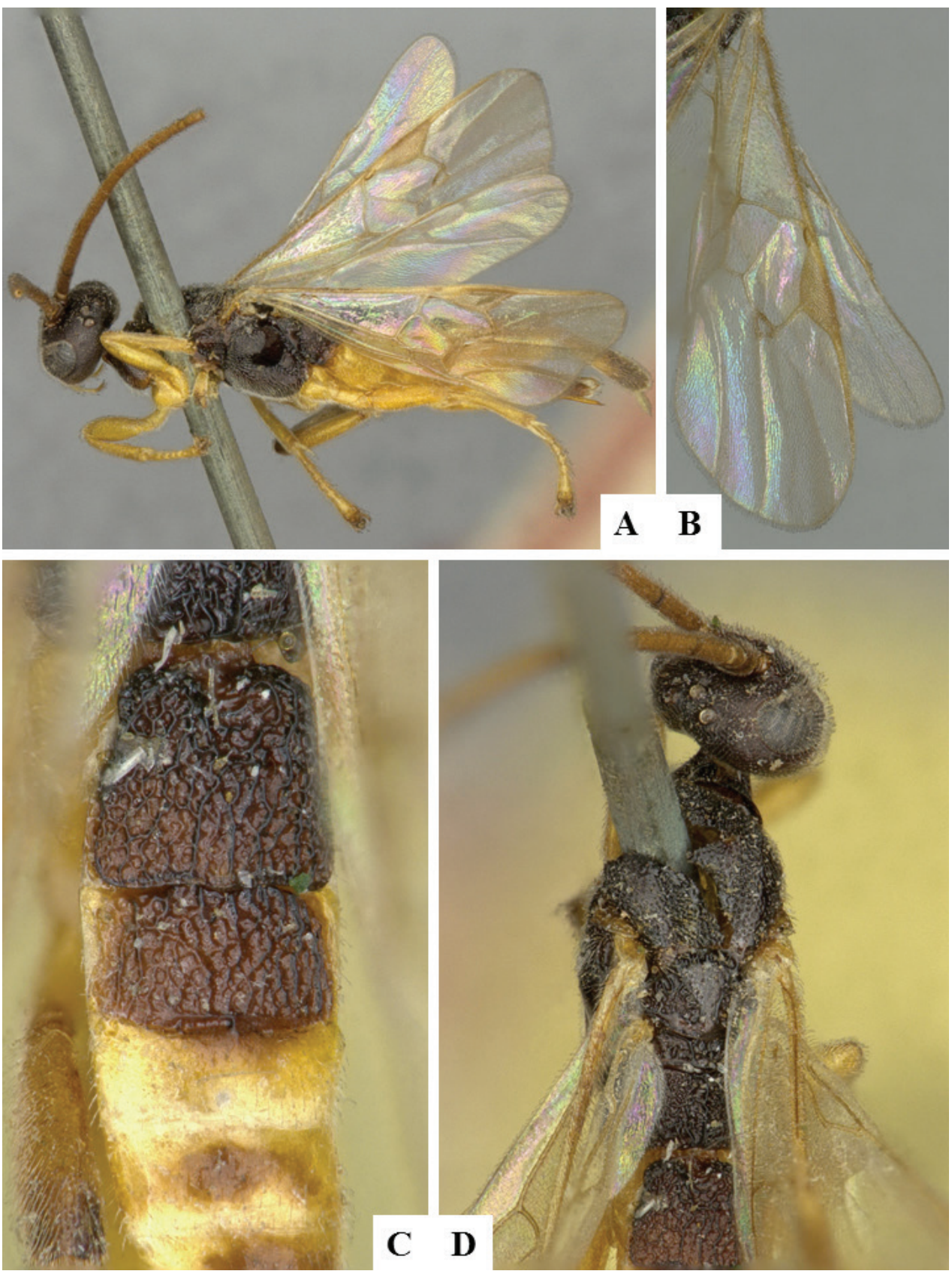

Figure 26. Hygroplitis melligaster. A Habitus, lateral B Wings C Metasoma (partially), dorsal D Head and mesosoma, dorsal. 
45.258675 -75.921130, 23.viii.1977, M. Sanborne, Voucher Code: CNC482267; 24.ix.1981, M.Sanborne, Voucher Code: CNC482265, CNC482266; 26.viii.1977, M. Sanborne, Voucher Code: CNC482268; Stittsville, 45.258675 -75.921130, 3.ix.1963, W. R. M. Mason, Voucher Code: CNC482263, CNC482264; Quebec, Gatineau Park, Camp Fortune, 45.509059 -75.851290, Malaise trap, 16.viii.1982, L. Masner, Voucher Code: CNC482269, CNC482270.

\section{Hypomicrogaster jft30}

Distribution. NEA.

Notes. This species corresponds in BOLD to BIN BOLD:AAD0217, with all specimens collected in southern Ontario.

Material examined. Ontario, Ottawa, city garden, $45.356100 \quad-75.707000$, 1.xi.2007, H. Goulet, Voucher Code: CAM0259.

\section{Hypomicrogaster jft31}

Distribution. NEA.

Notes. This species corresponds in BOLD to BIN BOLD:ACF0950, with all specimens collected in North America.

Material examined. Ontario, Woodlawn, 45.375000 -76.083000, 8.ix.2008, L. Masner, Voucher Code: MIC000623.

\section{Hypomicrogaster sp.}

\section{Distribution. NEA.}

Notes. This species corresponds in BOLD to BIN BOLD:AAD0218, with all specimens collected in eastern Canada. This seems to be another species related to $H$. zonaria (sensu Valerio and Whitfield 2015).

Material examined. Ontario, Ottawa, city garden, $45.356100-75.707000$, 1.ix.2007, H. Goulet, Voucher Code: CAM0267.

\section{Illidops sp. jft 18}

\section{Distribution. NEA.}

Notes. This is the only specimen of this genus known to us from Ottawa.

Material examined. Ontario, Constance Bay, 45.486308 -76.073461, 30.v.1939, G. S. Walley, Voucher Code: CNCHYM01550. 


\section{Lathrapanteles heleios Williams, 1985}

Fig. 27

\section{Distribution. NEA.}

Notes. The status of this species as a potential member of the Species Candidate Lists of COSEWIC was assessed by Fernandez Triana (2014).

Material examined. Ontario, Aylmer West, 45.400000 -75.850000, 27.viii.1972, Voucher Code: CNCHYM01571, CNCHYM01574; Mer Bleue, Ottawa, 45.393578 -75.512127, 10.vi.1975, H.J. Teskey, , 45.393585 -75.512138, 10.vi.1975, H. J. Teskey, Voucher Code: cnc482327, cnc482328, cnc482329, cnc482330, cnc482331, cnc482332, cnc482333, cnc482334, cnc482335, cnc482336, cnc482337, cnc482338, cnc482339, cnc482340, cnc482341, cnc482342, cnc482343, cnc482344, cnc482345, cnc482346; 45.393593 -75.512138, 10.vi.1975, H. J. Teskey, Voucher Code: CNCHYM01570, CNCHYM01573; Mer Bleue, 45.393578 -75.512127, 10.vi.1975, Teskay, H.J. , Voucher Code: CNC281023; Ottawa, city garden, 45.3561 -75.7069, 10.viii-1.ix.2007, H. Goulet, Voucher Code: CAM0895, CAM0903, CAM0904, CAM0905; 45.3561 -75.707, 1.ix.2007, H. Goulet, Voucher Code: CAM0266.

\section{Lathrapanteles papaipemae (Muesebeck, 1921)}

\section{Distribution. NEA.}

Material examined. Ontario, Aylmer West, 45.400000 -75.850000, Malaise trap, -, 27.viii.1972, Voucher Code: CNCHYM01576, CNCHYM01575.

\section{Microgaster canadensis Muesebeck, 1922}

\section{Distribution. NEA.}

Material examined. Ontario, Constance Bay, 45.486218 -76.073461, 21.vii.1933, G. S. Walley, Voucher Code: cnc482347; Corkery, 45.284686 -76.102742, 3.vii.1948, F.I.S., Voucher Code: CNC482348.

\section{Microgaster gelechiae Riley, 1869}

Fig. 28

\section{Distribution. NEA.}

Material examined. Ontario, Jockvale, 45.266494 -75.74418, 8.v.1943, J. McDunmough, Voucher Code: CNCHYM01616; 45.266524 -75.744180, 8.v.1943, McDunnough, J., Voucher Code: CNC281031; Ottawa, 45.382500 -75.713700, 1.v.1975, Voucher Code: CNCHYM01615. 

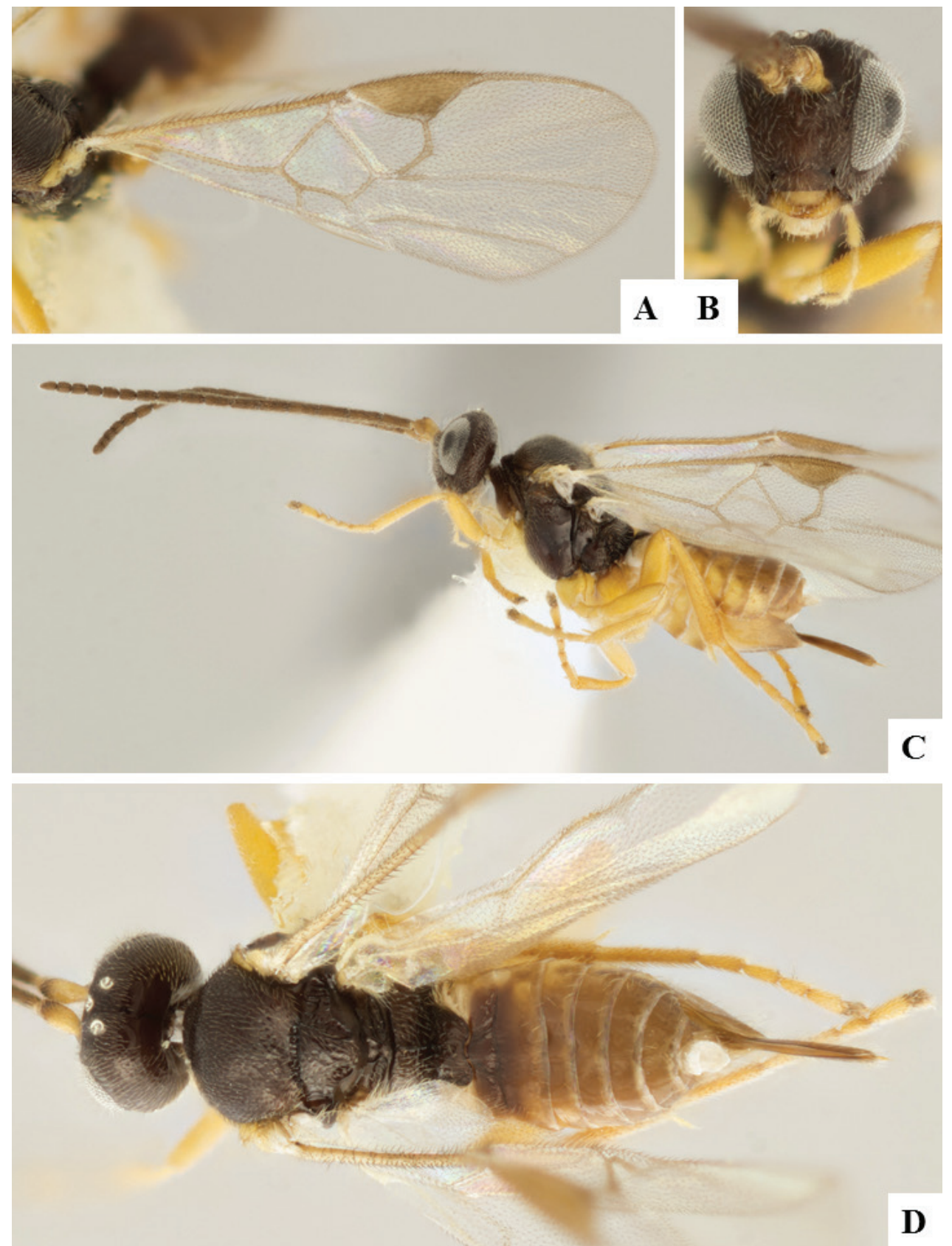

Figure 27. Lathrapanteles heleios. A Wings B Head, frontal C Habitus, lateral D Habitus, dorsal. 

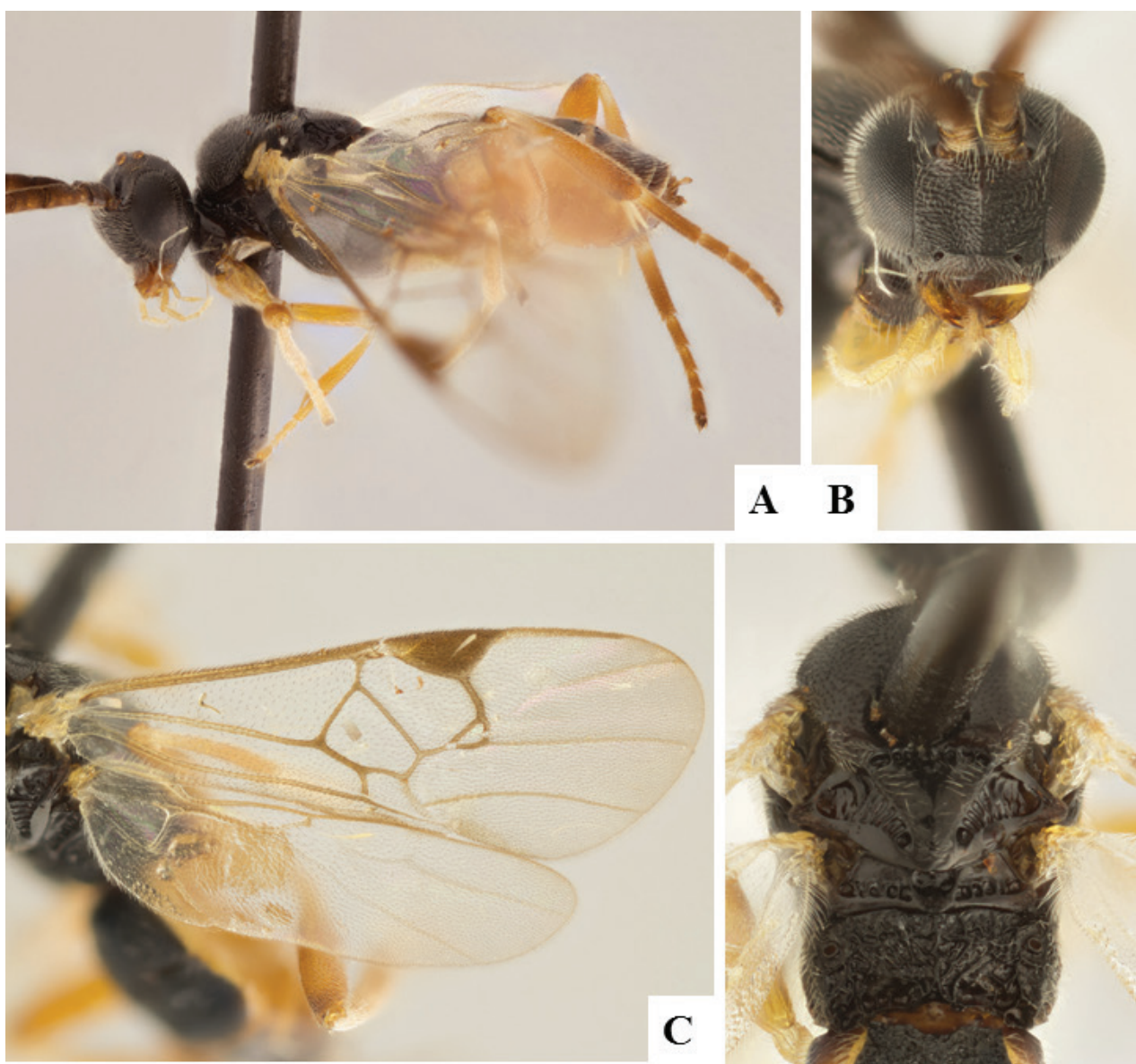

\section{A B}

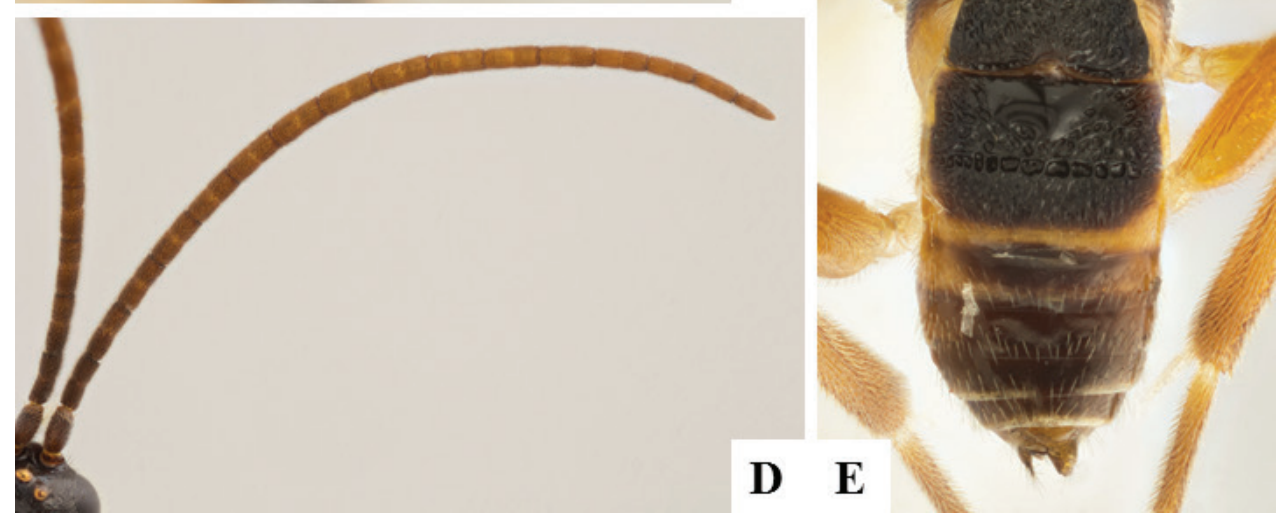

Figure 28. Microgaster gelechiae. A Habitus, lateral B Head, frontal C Wings D Antenna E Mesosoma and metasoma, dorsal. 


\section{Microgaster leechi Walley, 1935}

Fig. 29

\section{Distribution. NEA.}

Material examined. Ontario, Innisville, 45.054942 -76.250619, 6.viii.1963, W. R. M. Mason, Voucher Code: CNCHYM01630; 45.055468 -76.250497, 28.vii.1963, W. R. M. Mason, Voucher Code: CNC482351; Merivale, 45.325948 -75.719082, 25.vii.1930, J. J. deGryse, Voucher Code: CNC482349; Ottawa, 45.406631 -75.701407, 27.vii.1947, W. R. M. Mason, Voucher Code: CNC482353; Stittsville, 45.258675 -75.921130, 22.viii.1963, W. R. M. Mason, Voucher Code: CNC482350; 45.258796 -75.92113, 3.ix.1963, W. R. M. Mason, Voucher Code: CNCHYM01631; Quebec, Hull, 45.428550 -75.714554, 16.viii.1894, Voucher Code: CNC482352.

\section{Microgaster jft09}

\section{Distribution. NEA.}

Notes. This species corresponds in BOLD to BIN BOLD:ABZ2766, with all specimens collected in Canada.

Material examined. Ontario, $5 \mathrm{~km} \mathrm{NW}$ of Almonte, Hwy 49, Burnt Land, Alvar Prov. Park, Almonte, 45.255 -76.14, 29.v.2008, Goulet \& Fernandez, Voucher Code: CAM0331; North Gower to Smith Falls, 1 km N of Rd 6 \& Montague Bdy Rd, 45.033 -75.9, 15.vi.2004, Bennett \& Barnes, Voucher Code: HYM00001332; Ottawa, city garden, 45.356 -75.707, 24-30.v.2007, H. Goulet, Voucher Code: CAM0934.

\section{Microgaster jft10}

\section{Distribution. NEA, PAL.}

Notes. Specimens from both Europe and North America have rendered similar DNA barcodes, but we have not been able to match them to any described species -it remains as undescribed for the time being. This species corresponds in BOLD to BIN BOLD:ACE8790.

Material examined. Ontario, Ottawa, city garden, 45.356 -75.707, 16-26. vi.2007, H. Goulet, Voucher Code: CAM0926, CAM0927, CAM0928; 8-16.vi.2007, H. Goulet, Voucher Code: CAM0922, CAM0923, CAM0925.

\section{Microgaster jft11}

\section{Distribution. NEA.}

Notes. This species corresponds in BOLD to BIN BOLD:AAH3530, with all specimens collected in Canada. 

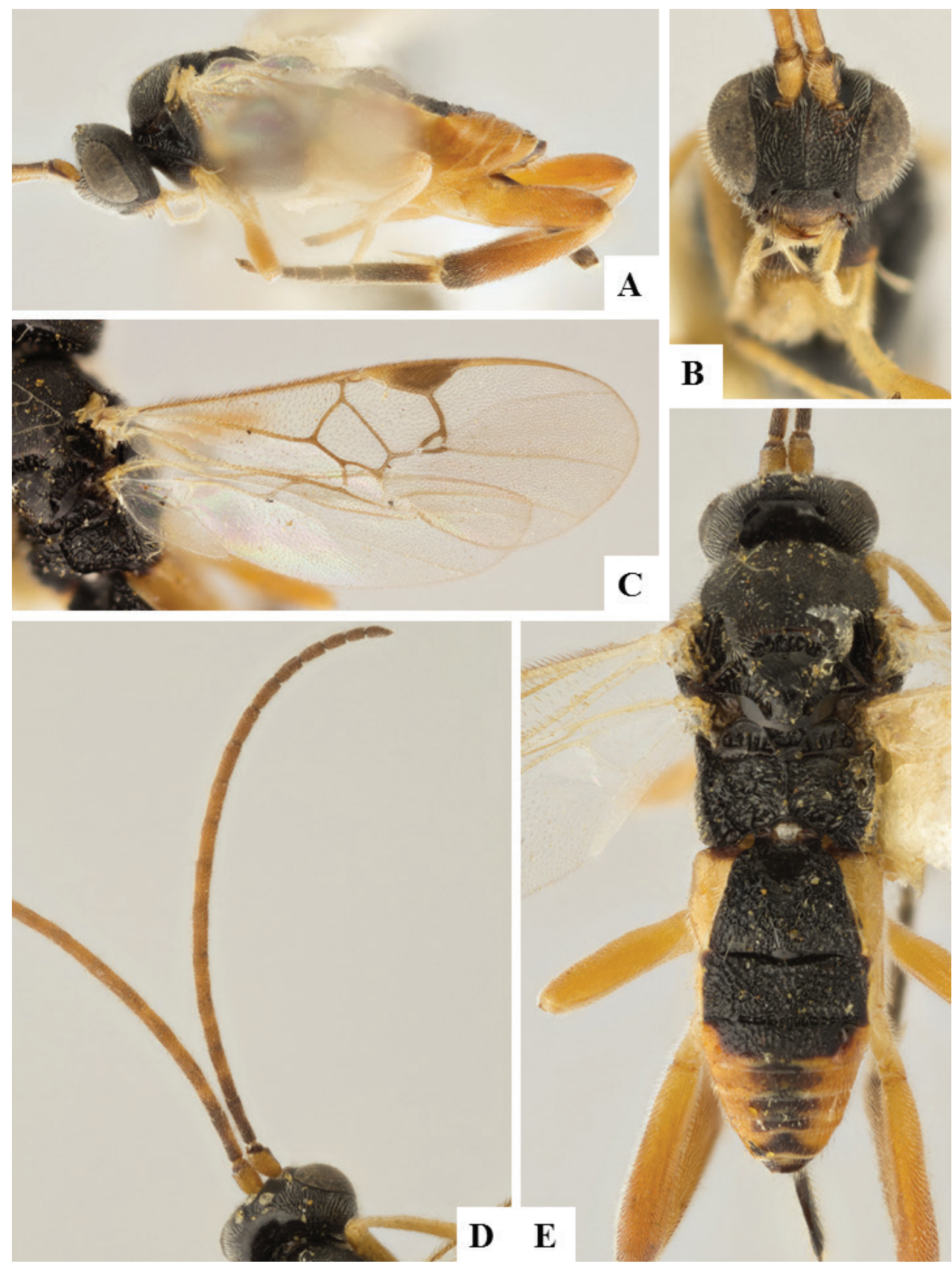

Figure 29. Microgaster leechi. A Habitus, lateral B Head, frontal C Wings D Antenna E Mesosoma and metasoma, dorsal.

Material examined. Ontario, Ottawa, city garden, 45.356 -75.707, 5.vi-2. vii.2008, H. Goulet, Voucher Code: CAM0920. 


\section{Microgaster jft14}

\section{Distribution. NEA.}

Notes. This species corresponds in BOLD to BIN BOLD:ABY6384, with all specimens collected in Canada.

Material examined. Ontario, Ottawa, city garden, 45.356 -75.707, 26.vi-13. vii.2007, H. Goulet, Voucher Code: CAM0931.

\section{Microgaster jft17}

\section{Distribution. NEA.}

Notes. This species corresponds in BOLD to BIN BOLD:AAA7886, with all specimens collected in North America. See comments on species jft21 below.

Material examined. Ontario, $2 \mathrm{~km} \mathrm{SW}$ of Innisville, 45.054942 -76.250619, 19.vi.1991, Denis \& Read, Voucher Code: GOU0259; 26.vi.1991, Denis \& Read, Voucher Code: GOU0273; mixed forest, 45.235 -75.624, 19-29.vi.2007, A. Bennett, Voucher Code: CAM0561; 29.vi-16.vii.2007, A. Bennett, Voucher Code: CAM0563; 7-19.vi.2007, A. Bennett, Voucher Code: CAM0562; Ottawa, city garden, 45.356 -75.707, 10.viii-1.ix.2007, H. Goulet, Voucher Code: CAM0919; 13-23.vii.2007, H. Goulet, Voucher Code: CAM0932; 24-30.v.2007, H. Goulet, Voucher Code: CAM0933; 30.vii-10.viii.2007, H. Goulet, Voucher Code: CAM0921.

\section{Microgaster jft21}

\section{Distribution. NEA.}

Notes. This species corresponds in BOLD to BIN BOLD:AAA7886, with all specimens collected in North America. Microgaster jft17 and jft 21 seem to be close (based on morphology and DNA barcodes), but there are also some differences among specimens, and thus we have decided to keep them as different species for the time being.

Material examined. Ontario, $2 \mathrm{~km} \mathrm{SW}$ of Innisville, 45.054942 -76.250619, 19.vi.1991, Denis \& Read, Voucher Code: GOU0260; Ottawa, city garden, 45.356 -75.707, 30.v-8.vi.2007, H. Goulet, Voucher Code: CAM0929; 8-16.vi.2007, H. Goulet, Voucher Code: CAM0924.

\section{Microgaster jft23}

\section{Distribution. NEA.}

Notes. This species corresponds in BOLD to BIN BOLD:AAB8447, with all specimens collected in North America. 
Material examined. Ontario, Ottawa, city garden, $45.356100-75.706900$, 26.vi13.vii.2007, H. Goulet, Voucher Code: CAM0930.

\section{Microgaster nr. epagoges}

Distribution. NEA.

Material examined. Ontario, Mer Bleue, Ottawa, 45.393594 -75.512139, 19.vii.1963, J. R. Vockeroth, Voucher Code: CNCHYM01608; Stittsville, 45.258797 -75.921133, 10.ix.1963, W. R. M. Mason, Voucher Code: CNCHYM01613.

\section{Microplitis autographae Muesebeck, 1922}

Distribution. NEA.

Material examined. Ontario, Hazeldean, 45.300928 -75.893728, 24.vi.1946, Voucher Code: CNCHYM01708; Ottawa, 45.3825 -75.7137, 26.iv.1948, A. R. Brooks, Voucher Code: CNCHYM01713; 45.382500 -75.713700, 27.vii.1947, W. R. M. Mason, Voucher Code: CNCHYM01717; Quebec, Hull, 45.428311 -75.713353, 2.v.1945, G. S. Walley, Voucher Code: CNCHYM01714.

\section{Microplitis gortynae Riley, 1881}

Distribution. NEA.

Material examined. Ontario, Innisville, 45.054942 -76.250619, 12.v.1963, W. R. M. Mason, Voucher Code: CNCHYM01752.

\section{Microplitis hyphantriae Ashmead, 1898}

Distribution. NEA.

Material examined. Ontario, Mer Bleue, Ottawa, 45.393585 -75.512138, 11.vii.1923, F. Ide, Voucher Code: CNC482354.

\section{Microplitis impressus (Wesmael, 1837)}

Distribution. NEA, PAL.

Material examined. Quebec, Chelsea, 45.541075 -75.867938, 24.iv.1933, G. S. Walley, Voucher Code: CNCHYM01763; 24.v.1933, G. S. Walley, Voucher Code: CNCHYM01764; 45.541315 -75.867938, 21.iv.1933, G. S. Walley, Voucher Code: CNC482361, CNC482362, CNC482363. 
Microplitis kewleyi Muesebeck, 1922

\section{Distribution. NEA.}

Material examined. Ontario, Ottawa, city garden, 45.3561 -75.7069, 1013.x.2008, H. Goulet, Voucher Code: WMIC0210; 10.viii-1.ix.2007, H. Goulet, Voucher Code: WMIC0204, WMIC0205, WMIC0206, WMIC0215, WMIC0217, WMIC0220; 20-29.ix.2008, H. Goulet, Voucher Code: WMIC0211; 30.vii-10. viii.2007, H. Goulet, Voucher Code: WMIC0196; Voucher Code: WMIC0208; 45.3561 -75.707, 10.viii.2007, H. Goulet, Voucher Code: CAM0169; 19.ix.2007, H. Goulet, Voucher Code: CAM0195.

\section{Microplitis maturus Weed, 1888}

Distribution. NEA.

Material examined. Ontario, Ottawa, 45.406631 -75.701407, Voucher Code: CNC482364.

\section{Microplitis plutellae Muesebeck, 1922}

Fig. 30

Distribution. NEA, OTL, PAL.

Material examined. Ontario, Ottawa, CNC breeding program, Ottawa, 45.3825 -75.7137, 31.iii.2008, Jose L. Fernandez Triana, Voucher Code: CPWH-0014, CPWH-0015, CPWH-0016, CPWH-0017, CPWH-0018, CPWH-0019, СРWH0020, CPWH-0021, CPWH-0022, СРWH-0023, СРWH-0024, СРWH-0025, CPWH-0026, CPWH-0027, СРWH-0028, СРWH-0029, СРWH-0030, СРWH0031, CPWH-0032; Ottawa, city garden, 45.356 -75.707, 10.viii-1.ix.2007, H. Goulet, Voucher Code: WMIC0212; Voucher Code: WMIC0192; Ottawa, 45.382500 -75.713700, 1953, D. Harcourt, Voucher Code: CNCHYM01833; 45.406631 -75.701407, 1953, D. Harcourt, Voucher Code: CNC482365, CNC482366, CNC482367, CNC482368, CNC482369.

\section{Microplitis scutellatus Muesebeck, 1922}

Distribution. NEA.

Material examined. Ontario, Carlsbad Springs, 45.369254 -75.456097, 5.iii.1941, Voucher Code: CNCHYM01845; Ottawa, 45.406631 -75.701407, Voucher Code: CNC482370. 

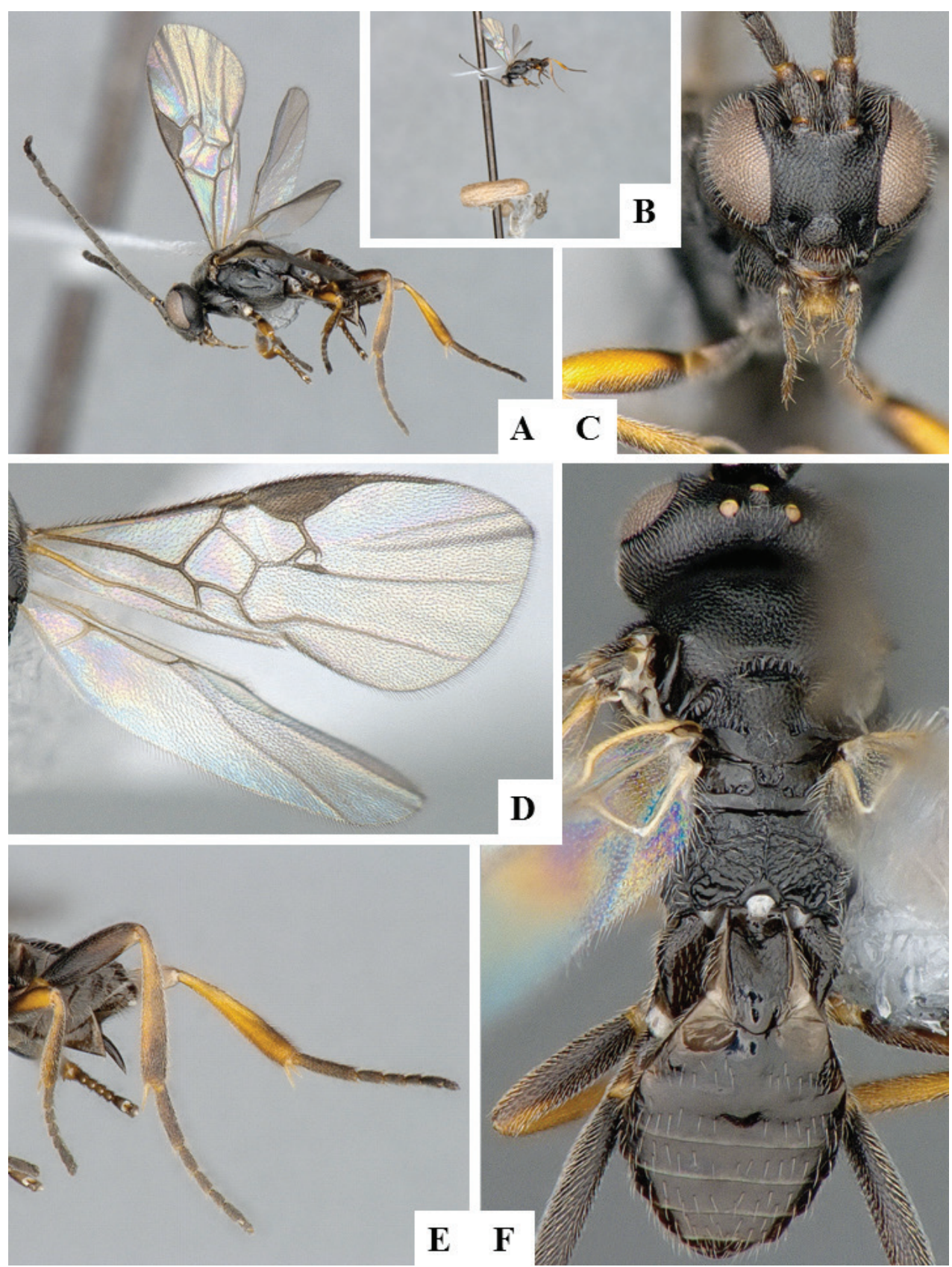

Figure 30. Microplitis plutellae. A Habitus, lateral B Glued specimen and cocoon C Head, frontal D Wings E Ovipositor sheaths E Habitus, dorsal. 


\section{Microplitis varicolor Viereck, 1917}

\section{Distribution. NEA.}

Notes. DNA barcodes of some specimens from North America identified as Microplits varicolor sometimes match DNA barcodes of some European specimens identified as $M$. mediator (Haliday, 1834). This topic needs to be investigated further, but for the time being we are considering the two species as separate.

Material examined. Ontario, $5 \mathrm{~km} \mathrm{NW}$ of Almonte, Hwy 49, Burnt Land, Alvar Prov. Park, Almonte, 45.2549 -76.14, 29.v.2008, Goulet \& Fernandez, Voucher Code: CAM0321; mixed forest, 45.2347 -75.624, 7-19.vi.2007, A. Bennett, Voucher Code: CAM0552; Ottawa, city garden, 45.356 -75.707, 28.vii.2009, L. Masner, Voucher Code: CNCH1023; 45.3561 -75.7069, 10.viii-1.ix.2007, H. Goulet, Voucher Code: WMIC0201, WMIC0203, WMIC0219, WMIC0222, WMIC0223, WMIC0224, WMIC0225, WMIC0226; 30.vii-10.viii.2007, H. Goulet, Voucher Code: WMIC0193, WMIC0194, WMIC0195, WMIC0197; 45.3561 -75.707, 10.viii.2007, H. Goulet, Voucher Code: CAM0091, CAM0093, CAM0094, CAM0095, CAM0170, CAM0171, CAM0172, CAM0173, CAM0175, CAM0176, CAM0177, CAM0178, CAM0248; 13.vii.2007, H. Goulet, Voucher Code: CAM0233; 16.v.2007, H. Goulet, Voucher Code: CAM0249, CAM0250; 16.vi.2007, H. Goulet, Voucher Code: CAM0191; 19.ix.2007, H. Goulet, Voucher Code: CAM0192, CAM0193, CAM0194, CAM0196, CAM0197, CAM0200, CAM0201, CAM0202; 19.x.2007, H. Goulet, Voucher Code: CAM0206, CAM0207, CAM0208, CAM0209; 30.v.2007, H. Goulet, Voucher Code: CAM0205, CAM0211, CAM0216, CAM0218, CAM0220, CAM0222, CAM0223, CAM0227; 30.vii.2007, H. Goulet, Voucher Code: CAM0180, CAM0182, CAM0183, CAM0185, CAM0186, CAM0187, CAM0188, CAM0189, CAM0190, CAM0235, CAM0236, CAM0237, CAM0239, CAM0240, CAM0241, CAM0243, CAM0244; 45.356100 -75.706900, H. Goulet, Voucher Code: WMIC0209; Ottawa, 45.406631 -75.701407, Dow's Swamp, 3.viii.1947, W. R. M. Mason, Voucher Code: CNC482371; Woodlawn, 45.3754 -76.0827, 6.viii.2008, L. Masner, Voucher Code: MIC000620.

\section{Microplitis jft07}

\section{Distribution. NEA.}

Notes. This species corresponds in BOLD to BIN BOLD:AAC0025, with all specimens collected in eastern Canada.

Material examined. Ontario, mixed forest, 45.2347 -75.624, 7-19.vi.2007, A. Bennett, Voucher Code: CAM0551. 


\section{Microplitis jft16}

\section{Distribution. NEA.}

Notes. This species corresponds in BOLD to BIN BOLD:AAA2409, with all specimens collected in North America.

Material examined. Ontario, Ottawa, Central Experimental Farm, 45.382500 -75.713700, 12.v.2000, Black \& Goulet, Voucher Code: GOU0219; Ottawa, city garden, 45.356 -75.707, 10.viii-1.ix.2007, H. Goulet, Voucher Code: WMIC0200, WMIC0216, WMIC0218; 10.viii.2007, H. Goulet, Voucher Code: CAM0092, CAM0174; 13.vii.2007, H. Goulet, Voucher Code: CAM0234; 19.ix.2007, H. Goulet, Voucher Code: CAM0198, CAM0199; 30.v.2007, H. Goulet, Voucher Code: CAM0203, CAM0204, CAM0210, CAM0212, CAM0213, CAM0215, CAM0224, CAM0225, CAM0226, CAM0229, CAM0230; 30.vii-10.viii.2007, H. Goulet, Voucher Code: WMIC0198, WMIC0199; 30.vii.2007, H. Goulet, Voucher Code: CAM0179, CAM0181, CAM0238, CAM0242, CAM0245, CAM0246, CAM0247; 45.356000 -75.707000, H. Goulet, Voucher Code: WMIC0207; 45.3561 -75.7069, H. Goulet, Voucher Code: WMIC0191; Woodlawn, 45.3754 -76.0827, 8.ix.2008, L. Masner, Voucher Code: MIC000618, MIC000621.

\section{Microplitis jft19}

\section{Distribution. NEA.}

Notes. This species corresponds in BOLD to BIN BOLD:AAD2718, with all specimens collected in Ottawa.

Material examined. Ontario, $5 \mathrm{~km}$ NW of Almonte, Hwy 49, Burnt Land, Alvar Prov. Park, Almonte, 45.255 -76.14, 29.v.2008, Goulet \& Fernandez, Voucher Code: CAM0320, CAM0322, CAM0324, CAM0325, CAM0327, CAM0328, CAM0329.

\section{Microplitis jft21}

\section{Distribution. NEA.}

Notes. This species corresponds in BOLD to BIN BOLD:AAE8603, with all specimens collected in eastern North America.

Material examined. Ontario, Ottawa, city garden, 45.356 -75.707, 30.v.2007, H. Goulet, Voucher Code: CAM0228, CAM0231; 30.vii.2007, H. Goulet, Voucher Code: CAM0184. 


\section{Microplitis jft23}

\section{Distribution. NEA.}

Notes. This species corresponds in BOLD to BIN BOLD:ABZ3353, with all specimens collected in southern Ontario.

Material examined. Ontario, Ottawa, city garden, 45.356 -75.707, 30.v.2007, H. Goulet, Voucher Code: CAM0221.

\section{Microplitis jft25}

\section{Distribution. NEA.}

Notes. This species corresponds in BOLD to BIN BOLD:AAF5357, with all specimens collected in southern Ontario.

Material examined. Ontario, Woodlawn, 45.375400 -76.082700, 8.ix.2008, L. Masner, Voucher Code: MIC000619.

\section{Microplitis jft34}

Distribution. NEA.

Notes. This species corresponds in BOLD to BIN BOLD:AAK6461, with all specimens collected in Canada.

Material examined. Ontario, Ottawa, city garden, $45.356-75.707$, 30.vii-10. viii.2007, H. Goulet, Voucher Code: WMIC0221.

\section{Microplitis jft47}

\section{Distribution. NEA.}

Notes. This species corresponds in BOLD to BIN BOLD:AAX4115, with all specimens collected in southern Ontario.

Material examined. Ontario, $5 \mathrm{~km} \mathrm{NW}$ of Almonte, Hwy 49, Burnt Land, Alvar Prov. Park, Almonte, 45.255 -76.14, 29.v.2008, Goulet \& Fernandez, Voucher Code: CAM0326.

\section{Microplitis jft50}

\section{Distribution. NEA.}

Notes. This species corresponds in BOLD to BIN BOLD:AAX4123, with all specimens collected in eastern Canada. 
Material examined. Ontario, mixed forest, 45.235 -75.624, 19-29.vi.2007, A. Bennett, Voucher Code: CAM0550.

\section{Microplitis jft52}

Distribution. NEA.

Notes. This species corresponds in BOLD to BIN BOLD:AAH6443, with all specimens collected in eastern Canada.

Material examined. Ontario, North Gower to Smith Falls, $1 \mathrm{~km} \mathrm{~N}$ of Rd 6 \& Montague Boundary Rd, 45.033 -75.9, 15.vi.2004, Bennett \& Barnes, Voucher Code: HYM00001235, HYM00001236.

\section{Microplitis jft53}

Distribution. NEA.

Notes. This species corresponds in BOLD to BIN BOLD:AAE8502, with all specimens collected in North America.

Material examined. Ontario, $5 \mathrm{~km} \mathrm{NW}$ of Almonte, Hwy 49, Burnt Land, Alvar Prov. Park, Almonte, 45.255 -76.14, 29.v.2008, Goulet \& Fernandez, Voucher Code: CAM0323, CAM0330; Ottawa, city garden, $45.356-75.707$, 30.v.2007, H. Goulet, Voucher Code: CAM0214.

\section{Microplitis jft56}

Distribution. NEA.

Notes. This species corresponds in BOLD to BIN BOLD:AAH3516, with all specimens collected in southern Ontario.

Material examined. Ontario, Ottawa, city garden, 45.356 -75.707, 30.v.2007, H. Goulet, Voucher Code: CAM0217, CAM0219.

\section{Microplitis jft60}

\section{Distribution. NEA.}

Notes. This species corresponds in BOLD to BIN BOLD:AAE8461, with all specimens collected in North America.

Material examined. Ontario, mixed forest, 45.235 -75.624, 29.vi-16.vii.2007, A. Bennett, Voucher Code: CAM0553; Ottawa, city garden, 45.356 -75.707, 10.viii-1. ix.2007, H. Goulet, Voucher Code: WMIC0202, WMIC0213. 


\section{Microplitis nr. autographae}

Distribution. NEA.

Material examined. Ontario, Ottawa, 45.406633 -75.701408, 27.vii.1947, W.R.M. Mason, Voucher Code: CNC483613.

\section{Pholetesor bedelliae (Viereck, 1911)}

Fig. 31

Distribution. NEA, NEO.

Material examined. Ontario, $5 \mathrm{~km}$ NW of Almonte, Hwy 49, Burnt Land, Alvar Prov. Park, Almonte, 45.255 -76.14, 29.v.2008, Goulet \& Fernandez, Voucher Code: CAM0437; Kinburn, 45.391853 -76.188798, 9.iii.1958, Freeman \& Lewis, Voucher Code: CNC482372; Ottawa, city garden, 45.356 -75.707, 30.v.2007, H. Goulet, Voucher Code: CAM0278.

\section{Pholetesor masoni Whitfield, 2006}

Distribution. NEA, NEO.

Material examined. Ontario, Aylmer West, 45.395345 -75.844876, Malaise trap, 30.v.1972, Voucher Code: CNC482374, CNC482375, CNC482376, CNC482377, CNC482378, CNC482379, CNC482380, CNC482381, CNC482382, CNC482383, CNC482384, CNC482385, CNC482386, CNC482387; Ottawa, 45.356083 -75.706933, 25.v.1944, McDunnough, J. , Voucher Code: CNC309874; 45.406631 -75.701407, 25.vii.1957, J. E. H. Martin, Voucher Code: CNC482373.

\section{Pholetesor nanus (Reinhard, 1880)}

Distribution. NEA, PAL.

Notes. This is the first record of this species for the Nearctic region.

Material examined. Ontario, Bells Corners, 45.322133 -75.833301, 25.vii.1962, Miller, C.D. Voucher Code: CNC309876.

\section{Pholetesor ornigis (Weed, 1887)}

Fig. 32

\section{Distribution. NEA.}

Material examined. Ontario, $5 \mathrm{~km}$ NW of Almonte, Hwy 49, Burnt Land, Alvar Prov. Park, Almonte, 45.2549 -76.14, 29.v.2008, Goulet \& Fernandez, Voucher Code: 
CAM0424, CAM0433; Aylmer, 45.395345 -75.844876, 18.vii.1960, C. D. Miller, Voucher Code: CNC482388, CNC482389, CNC482390; Bells Corners, 45.322247 -75.833249, 2.viii.1962, C. D. Miller, Voucher Code: CNC482396; 7.viii.1960, C. D. Miller, Voucher Code: CNC482395; Constance Bay, 45.486218 -76.073461, 4-5.viii.1955, T. N. Freeman, Voucher Code: CNC483512; 5.viii.1955, T. N. Freeman, Voucher Code: CNC482423, CNC483510, CNC483511; Fitzroy Harbour, 45.471703 -76.214077, 17.viii.1960, C.D. Miller, Voucher Code: CNC483564, CNC483565, CNC483566, CNC483567, CNC483568, CNC483569; 6.vi.1962, Freeman \& Lewis, Voucher Code: CNC483571, CNC483572, CNC483573, CNC483574, CNC483575, CNC483576, CNC483577, CNC483578; 8-9.vi.1962, Freeman \& Lewis, Voucher Code: CNC483580; 9.vi.1962, Freeman \& Lewis, Voucher Code: CNC483579; Kemptville, 45.016409 -75.646449, 12.iii.1962, Voucher Code: CNC483582; 13.iii.1962, Voucher Code: CNC483583; 19.iii.1962, Voucher Code: CNC483584; Ottawa, 45.406631 -75.701407, 18.i.1961, Freeman \& Lewis, Voucher Code: CNC482412; 2.vii.1960, J.R. Vockeroth, Voucher Code: CNC483581; 22.i.1961, Freeman \& Lewis, Voucher Code: CNC482415, CNC482422; 25.i.1961, Freeman \& Lewis, Voucher Code: CNC482410, CNC482411; 3.ii.1961, Freeman \& Lewis, Voucher Code: CNC482413; 5.ii.1961, Freeman \& Lewis, Voucher Code: CNC482414; Stittsville, 45.258675 -75.921130, 11.vii.1960, Voucher Code: CNC482406; 12.vii.1960, Voucher Code: CNC482405; 13.vii.1960, Voucher Code: CNC482403; 18.vii.1960, Voucher Code: CNC482399, CNC482400, CNC482401, CNC482402; 19.vii.1960, Voucher Code: CNC482404; 20.vii.1960, C. D. Miller, Voucher Code: CNC482397, CNC482398; 22.vii.1960, C.D. Miller, - , Voucher Code: CNC483570, CNC482407; 23.vii.1960, Voucher Code: CNC482408; 25.vii.1960, Voucher Code: CNC482409; Woodlawn, 45.462683 -76.075555, 14.viii.1962, C. D. Miller, Voucher Code: CNC482419; Quebec, Aylmer, 45.395345 -75.844876, 12.vii.1960, C. D. Miller, Voucher Code: CNC482391, CNC482392, CNC482393, CNC482394; Gatineau Park, 45.60057 -76.042647, 24.v.2007, L. Masner, Voucher Code: HYM00001323; Gatineau, 45.478218 -75.701346, 2.viii.1960, C. D. Miller, Voucher Code: CNC482426, CNC483509; 29.vii.1960, C. D. Miller, Voucher Code: CNC483507; 3-8.viii.1960, C. D. Miller, Voucher Code: CNC482427; 3.viii.1960, C. D. Miller, Voucher Code: CNC482428, CNC482430, CNC483506; 31.vii.1960, C. D. Miller, Voucher Code: CNC482433, CNC483508; 4.viii.1960, C. D. Miller, Voucher Code: CNC482429; 8.viii.1960, C. D. Miller, Voucher Code: CNC482425, CNC482431, CNC482432; 9.viii.1960, C. D. Miller, Voucher Code: CNC482424; Hull, 45.428550 -75.714554, 10.viii.1962, C. D. Miller, Voucher Code: CNC482451; 11.viii.1962, C. D. Miller, Voucher Code: CNC483505; 11.viii.1965, C. D. Miller, Voucher Code: CNC483549; 12.viii.1960, C. D. Miller, Voucher Code: CNC483532, CNC483533; 13.v.1963, C. D. Miller, Voucher Code: CNC483521, CNC483522, CNC483523; 13.viii.1962, C. D. Miller, Voucher Code: CNC482450, CNC483501; 14.vii.1962, C. D. Miller, Voucher Code: CNC483518; 14.viii.1962, C. D. Miller, Voucher Code: CNC483519, CNC483520; 15.v.1963, C. D. Miller, Voucher Code: CNC482448; 16.viii.1962, C. D. Miller, Voucher Code: CNC483498; 17.vii.1963, C. 

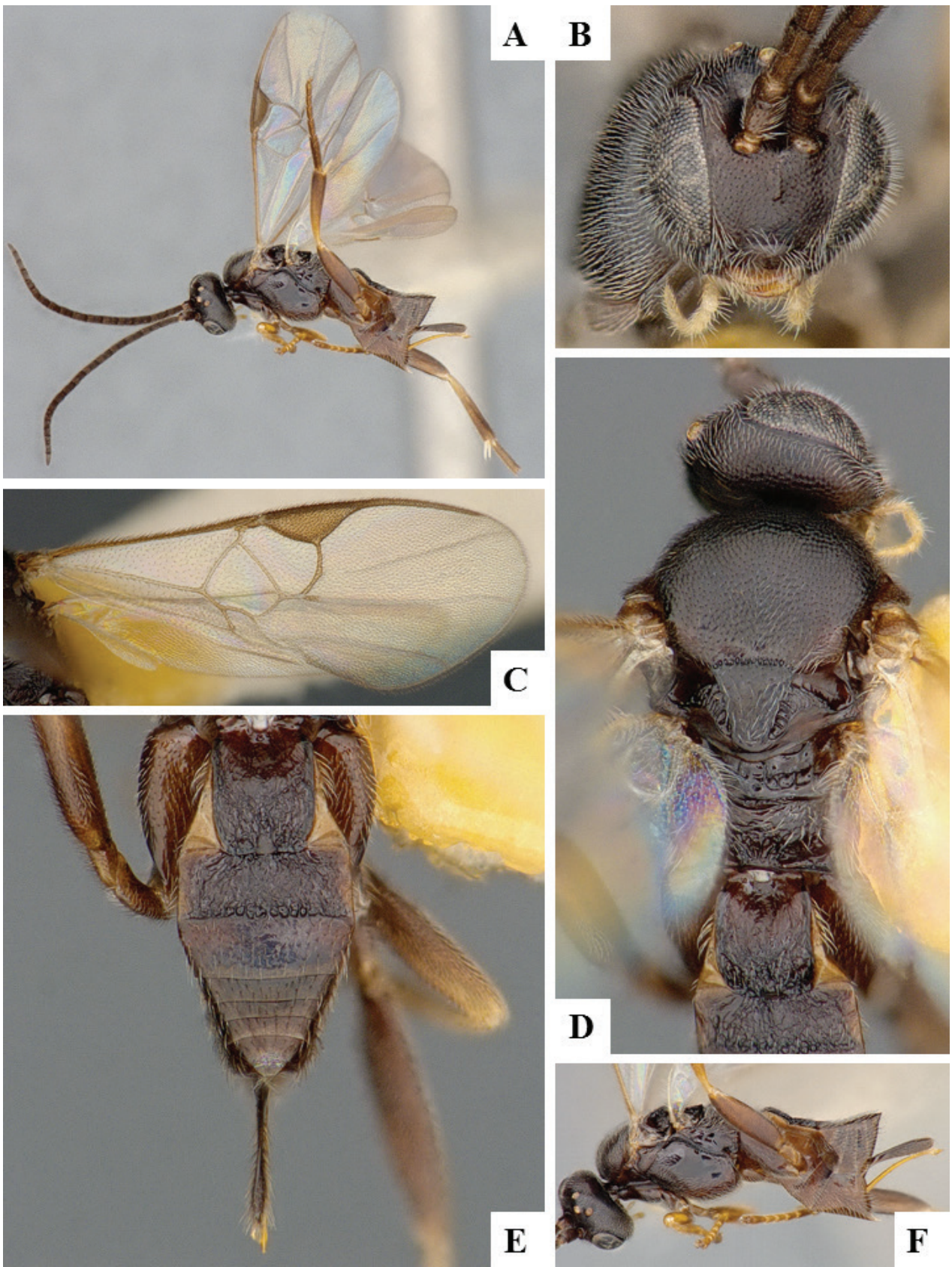

Figure 3I. Pholetesor bedelliae. A Habitus, lateral B Head, frontal C Wings D Head and mesosoma, dorsal E Metasoma, dorsal F Mesosoma and metasoma, lateral. 

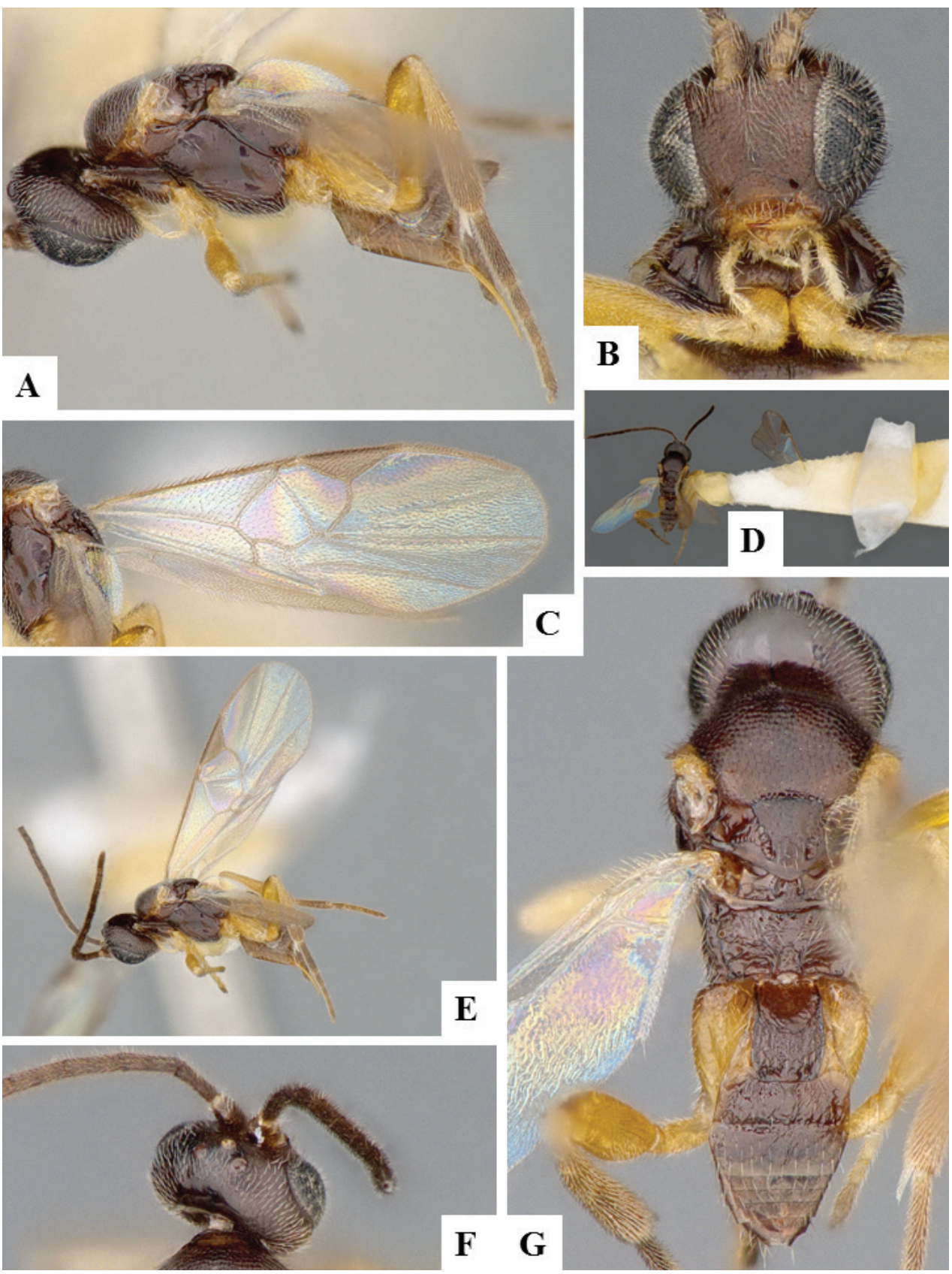

Figure 32. Pholetesor ornigis. A Head and mesosoma, lateral B Head, frontal C Wings D Glued specimen and cocoon E Habitus, lateral F Head, dorsal G Habitus, dorsal. 
D. Miller, Voucher Code: CNC483513, CNC483514, CNC483515, CNC483516, CNC483517; 17.viii.1962, C. D. Miller, Voucher Code: CNC483497; 2.viii.1960, C. D. Miller, Voucher Code: CNC483525, CNC483551, CNC483552, CNC483553, CNC483554, CNC483555, CNC483562, CNC483563; 2.viii.1962, C. D. Miller, Voucher Code: CNC482447; 23.viii.1965, C. D. Miller, Voucher Code: CNC483536; 27.vii.1960, C. D. Miller, Voucher Code: CNC483529, CNC483530; 28.vii.1960, C. D. Miller, Voucher Code: CNC483526; 28.viii.1962, C. D. Miller, Voucher Code: CNC483503; 29.vii.1960, C. D. Miller, Voucher Code: CNC483528; 3.viii.1960, C. D. Miller, Voucher Code: CNC483556, CNC483557, CNC483558, CNC483559; 3.viii.1962, C. D. Miller, Voucher Code: CNC482440, CNC482441, CNC482442, CNC482449, CNC483543, CNC483544; 30.vii.1962, C. D. Miller, Voucher Code: CNC483502; 30.viii.1962, C. D. Miller, Voucher Code: CNC482437, CNC482438, CNC482439, CNC483495, CNC483496, CNC483499, CNC483504; 31.vii.1960, C. D. Miller, Voucher Code: CNC483561; 31.vii.1962, C. D. Miller, Voucher Code: CNC483524; 31.viii.1965, C. D. Miller, Voucher Code: CNC483537, CNC483550; 4.iii.1957, Freeman \& Lewis, Voucher Code: CNC482434, CNC482435; 4.viii.1960, C. D. Miller, Voucher Code: CNC483527; 4.viii.1965, C. D. Miller, Voucher Code: CNC483545; 5.viii.1960, C. D. Miller, Voucher Code: CNC483531, CNC483560; 6.viii.1965, C. D. Miller, Voucher Code: CNC483535, CNC483546, CNC483547, CNC483548; 7.viii.1962, C. D. Miller, Voucher Code: CNC482443, CNC482444, CNC482445, CNC482446, CNC483538, CNC483539, CNC483540, CNC483541, CNC483542; 8.viii.1960, C. D. Miller, Voucher Code: CNC483534; 8.xi.1960, T. N. Freeman, Voucher Code: CNC482436; 9-30.viii.1962, C. D. Miller, Voucher Code: CNC483500; Val Tetreau, 45.419217 -75.745275, 18.i.1961, Freeman \& Lewis, Voucher Code: CNC482420; 27.i.1961, Freeman \& Lewis, Voucher Code: CNC482421; 8.viii.1960, C. D. Miller, Voucher Code: CNC482416, CNC482417, CNC482418.

\section{Pholetesor pinifoliellae Whitfield, 2006}

\section{Distribution. NEA.}

Material examined. Ontario, Ottawa, 45.356083 -75.706933, 28.vii.1950, Voucher Code: CNC Type 19982 and one additional specimen without any voucher code.

\section{Pholetesor rhygoplitoides Whitfield, 2006}

Fig. 33

\section{Distribution. NEA.}

Material examined. Ontario, Mer Bleue, 45.393578 -75.512127, 19.vii.1963, Chillcott, J.G, Voucher Code: CNC309878; 45.393593 -75.512138, 18.vii.1963, J. G. Chillcott, Voucher Code: CNCHYM03189. 
Pholetesor salicifoliellae Mason, 1959

Fig. 34

\section{Distribution. NEA.}

Material examined. Ontario, $5 \mathrm{~km} \mathrm{NW}$ of Almonte, Hwy 49, Burnt Land, Alvar Prov. Park, Almonte, 45.2549 -76.14, 29.v.2008, Goulet \& Fernandez, Voucher Code: CAM0343, CAM0355, CAM0362, CAM0420, CAM0 425.

\section{Pholetesor viminetorum (Wesmael, 1837)}

Distribution. NEA, PAL.

Material examined. Ontario, $5 \mathrm{~km} \mathrm{NW}$ of Almonte, Hwy 49, Burnt Land, Alvar Prov. Park, Almonte, 45.255 -76.14, 29.v.2008, Goulet \& Fernandez, Voucher Code: CAM0365, CAM0414, CAM0415, CAM0416, CAM0417, CAM0418, CAM0419, CAM0421, CAM0422, CAM0423, CAM0426, CAM0427, CAM0428, CAM0429, CAM0430, CAM0431, CAM0432, CAM0434, CAM0435, CAM0438.

\section{Pholetesor jft03}

\section{Distribution. NEA.}

Notes. This species corresponds in BOLD to BIN BOLD:ABZ3171, with all specimens collected in eastern North America.

Material examined. Ontario, Ottawa, city garden, 45.3561 -75.7069, 30.vii-10. viii.2007, H. Goulet, Voucher Code: CAM0900.

\section{Pholetesor jft04}

\section{Distribution. NEA.}

Notes. This species corresponds in BOLD to BIN BOLD:AAE0349, with all specimens collected in eastern Canada.

Material examined. Ontario, Ottawa, city garden, 45.3561 -75.7069, 8-16. vi.2007, H. Goulet, Voucher Code: CAM0896; 45.3561 -75.707, 23.vii.2007, H. Goulet, Voucher Code: CAM0283, CAM0284; 30.v.2007, H. Goulet, Voucher Code: CAM0279; 8.vi.2007, H. Goulet, Voucher Code: CAM0285; 45.356100 -75.706900, 30.vii-10.viii.2007, H. Goulet, Voucher Code: CAM0901.

\section{Pholetesor jft05}

Distribution. NEA. 

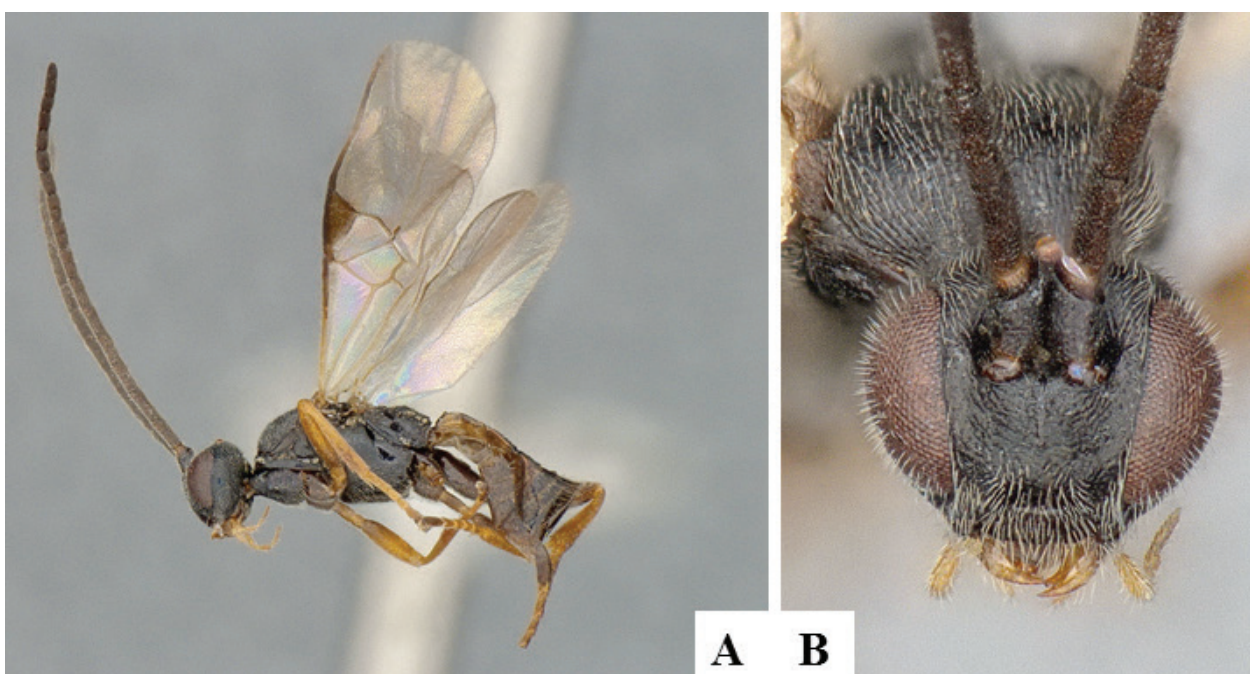

A B
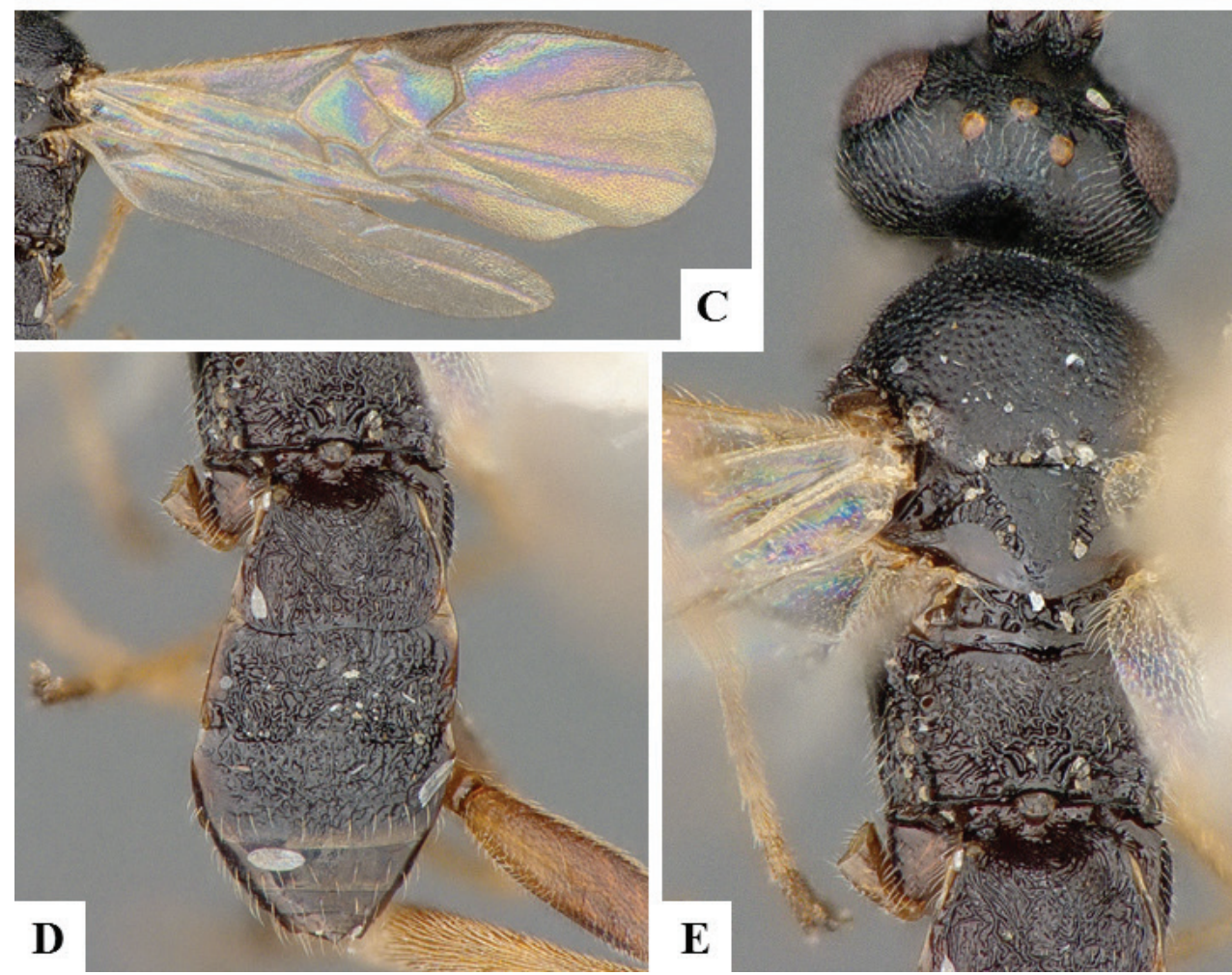

Figure 33. Pholetesor rhygoplitoides. A Habitus, lateral B Head, frontal C Wings D Metasoma, dorsal E Head and mesosoma, dorsal. 

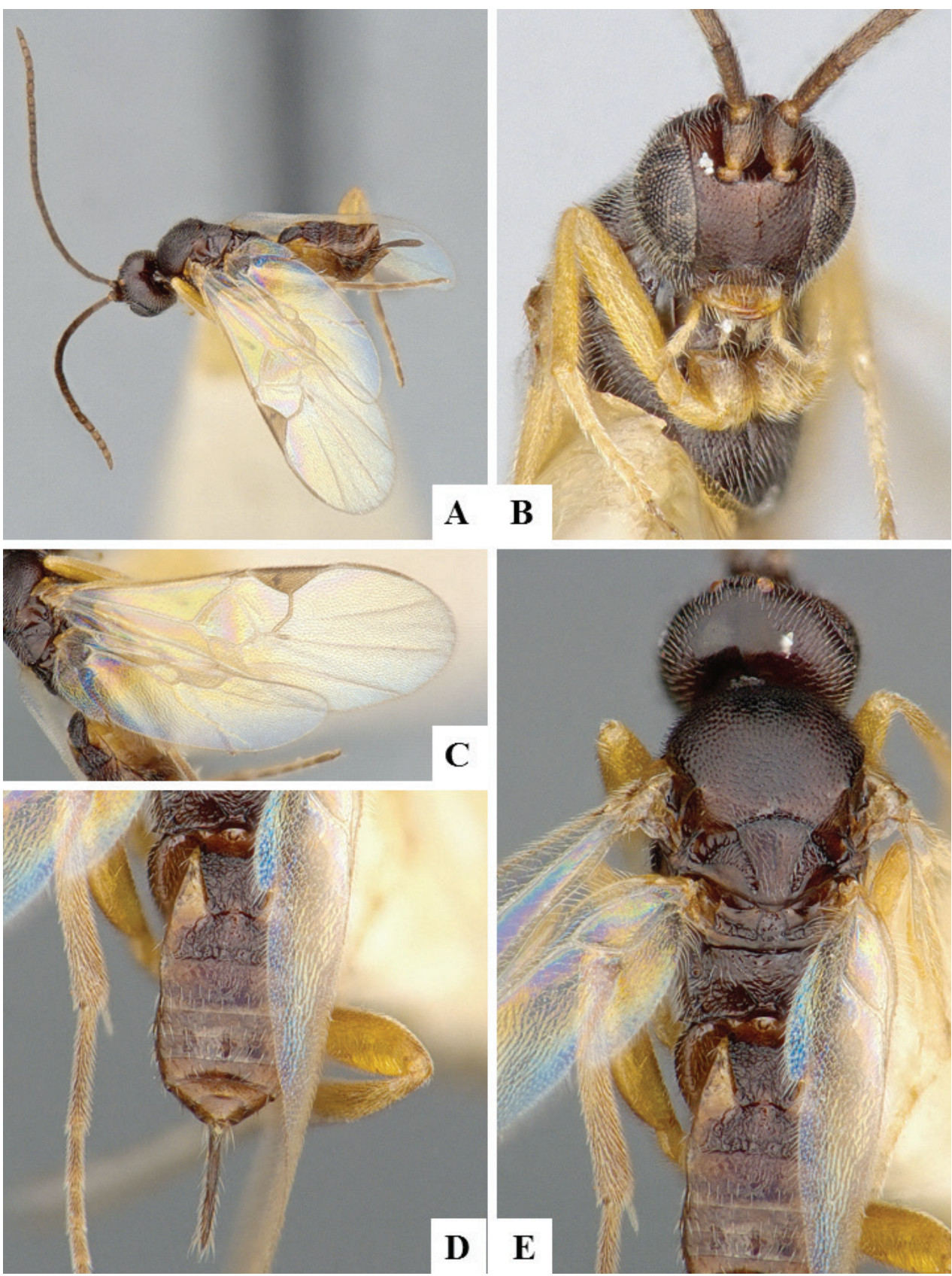

Figure 34. Pholetesor salicifoliellae. A Habitus, lateral B Head, frontal C Wings D Metasoma, dorsal E Head and mesosoma, dorsal. 
Notes. This species corresponds in BOLD to BIN BOLD:AAD5198, with all specimens collected in North America.

Material examined. Ontario, Ottawa, city garden, $45.3561-75.707$, 1.ix.2007, H. Goulet, Voucher Code: CAM0281; 16.vi.2007, H. Goulet, Voucher Code: CAM0002, CAM0004; 23.vii.2007, H. Goulet, Voucher Code: CAM0282.

\section{Pholetesor jft09}

\section{Distribution. NEA.}

Notes. This species corresponds in BOLD to BIN BOLD:AAA8732, with all specimens collected in North America.

Material examined. Ontario, Ottawa, city garden, $45.356100-75.707000$, 8.ix.2007, H. Goulet, Voucher Code: CAM0100.

\section{Pholetesor jft12}

Distribution. NEA.

Notes. This species corresponds in BOLD to BIN BOLD:AAB5026, with all specimens collected in Canada.

Material examined. Ontario, Ottawa, city garden, 45.356100 -75.706900, 2330.vii.2007, H. Goulet, Voucher Code: CAM0899.

\section{Protapanteles paleacritae (Riley, 1881)}

Fig. 35

\section{Distribution. NEA.}

Material examined. Ontario, Carp, 45.349117 -76.041225, 23.vii.1942, Voucher Code: CNC483585; Greely, 45.259544 -75.568696, 25.vi.1946, Voucher Code: CNC483586.

Protapanteles phlyctaeniae (Muesebeck, 1929)

Fig. 36

Distribution. NEA.

Material examined. Ontario, Ottawa, 45.3825-75.7137, 18.vii.1950, E. Munroe, Voucher Code: CNCHYM03246. 


\section{Protapanteles $\mathrm{cf}$. anchisiades}

Distribution. NEA, PAL.

Notes. This species seems to be part of a complex related to Protapanteles anchisiades (Nixon, 1973), from both European and North American specimens. For the time being is left as an undescribed species, until more studies of the Holarctic fauna are carried out. This species corresponds in BOLD to BIN BOLD:AAA7147.

Material examined. Ontario, Ottawa, city garden, 45.356100 -75.706900, 23.vii.2009, L. Masner, Voucher Code: CNCH0461.

\section{Protapanteles jft02}

\section{Distribution. NEA, PAL.}

Notes. This species seems to be part of a complex related to Protapanteles anchisiades (Nixon, 1973), from both European and North American specimens. For the time being is left as an undescribed species, until more studies of the Holarctic fauna are carried out. This species corresponds in BOLD to BIN BOLD:AAA7147.

Material examined. Ontario, Ottawa, city garden, 45.3561 -75.7069, 16.vi.2007, H. Goulet, Voucher Code: HYM00000998; 45.3561 -75.707, 1.ix.2007, H. Goulet, Voucher Code: CAM0269; 1.xi.2007, H. Goulet, Voucher Code: CAM0271; 30.vii.2007, H. Goulet, Voucher Code: CAM0275.

\section{Protomicroplitis calliptera (Say, 1836)}

Fig. 37

Distribution. NEA.

Notes. The status of this species as a potential member of the Species Candidate Lists of COSEWIC was assessed by Fernandez Triana (2014).

Material examined. Ontario, Stittsville, 45.258675 -75.921130, 27.viii.1963, W.R.M. Mason, Voucher Code: CNC483587; 30.vi.1963, W.R.M. Mason, Voucher Code: CNC483588; 45.258796 -75.92113, 10.ix.1963, W. R. M. Mason, Voucher Code: CNCHYM03255; 45.259039 -75.920958, 10.ix.1963, Mason, W.R.M, Voucher Code: CNC309895.

\section{Pseudapanteles gouleti Fernandez-Triana, 2010}

Fig. 38

\section{Distribution. NEA.}

Notes. The status of this species as a potential member of the Species Candidate Lists of COSEWIC was assessed by Fernandez Triana (2014). 


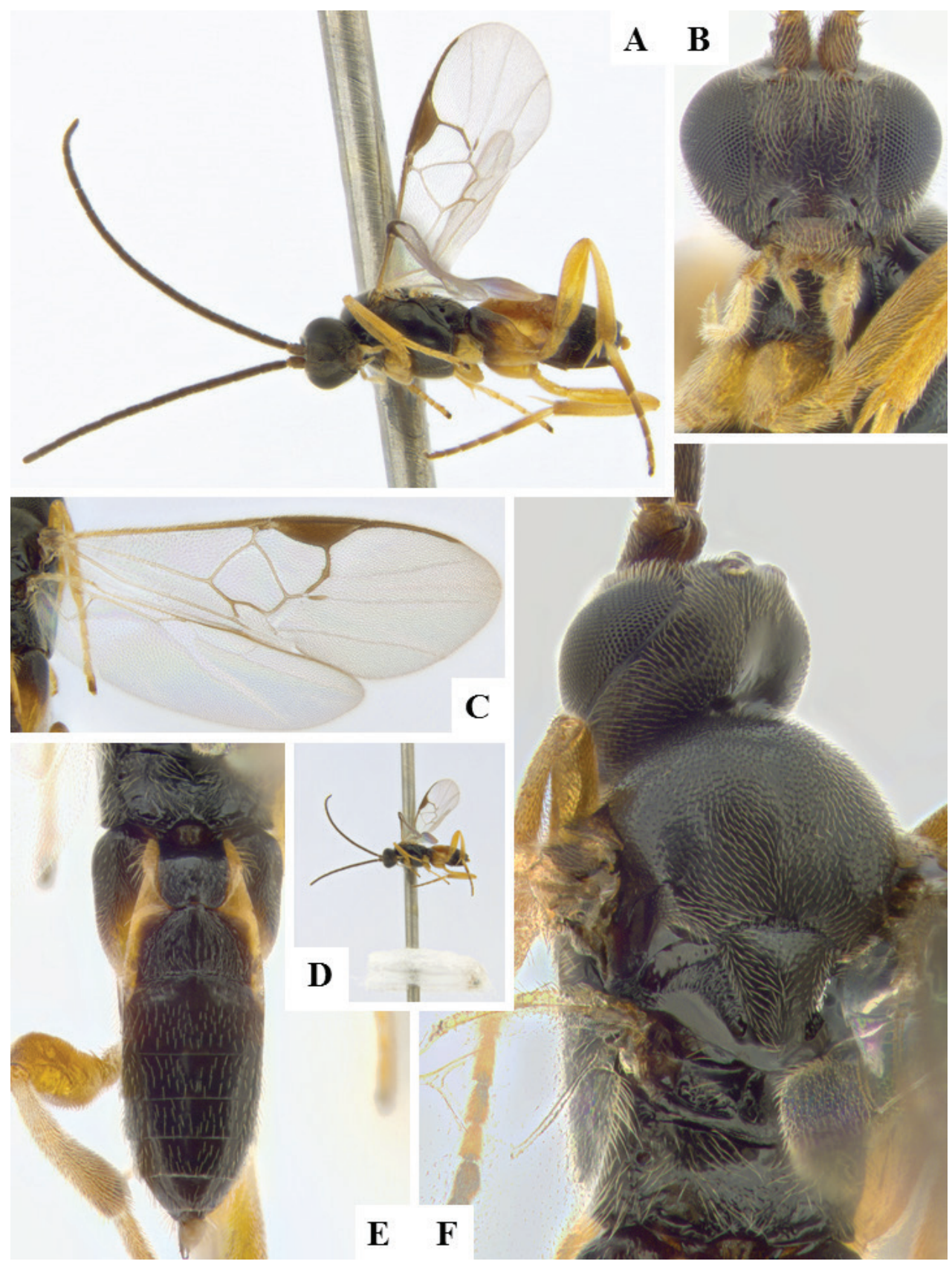

Figure 35. Protapanteles paleacritae. A Habitus, lateral B Head, frontal C Wings D Glued specimen and cocoon E Metasoma, dorsal F Head and mesosoma, dorsal. 


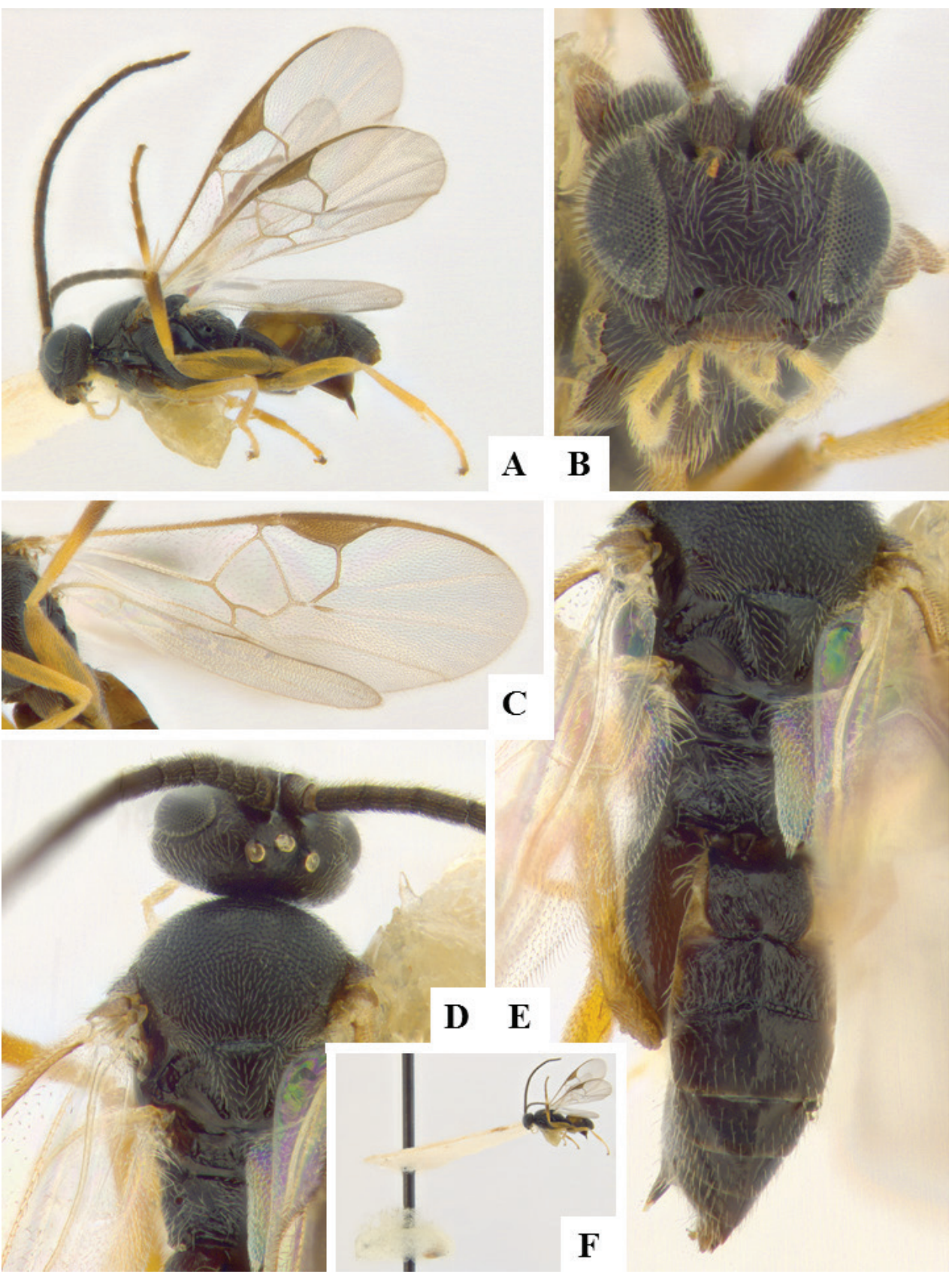

Figure 36. Protapanteles phlyctaeniae. A Habitus, lateral B Head, frontal C Wings D Head and mesosoma, dorsal E Mesosoma (partially) and metasoma, dorsal F Glued specimen and cocoon. 

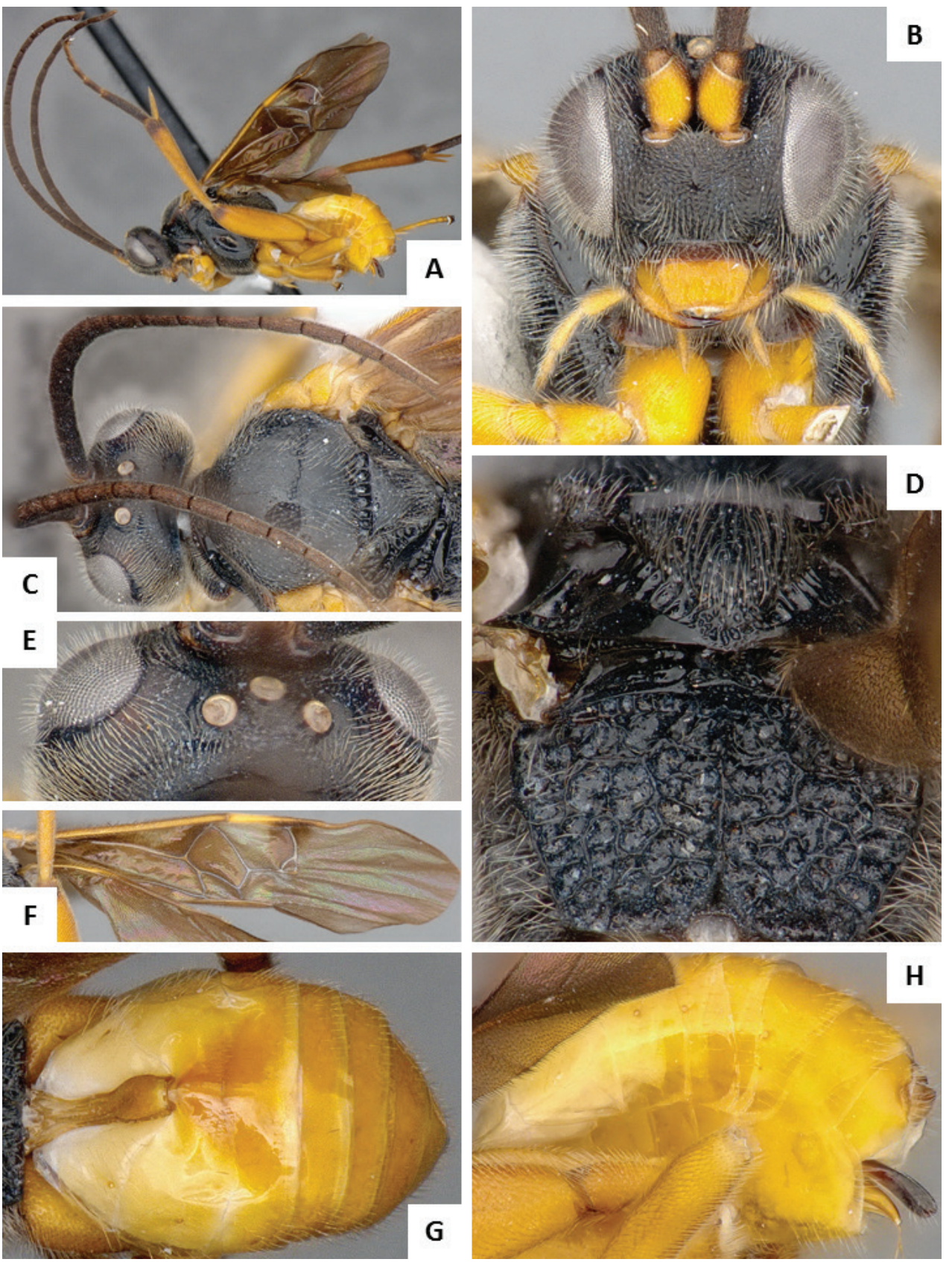

Figure 37. Protomicroplitis calliptera. A Habitus, lateral B Head, frontal C Head and mesosoma (partially), dorsal D Propodeum E Head, dorsal F Wings G Metasoma, dorsal $\mathbf{H}$ Metasoma, lateral. 

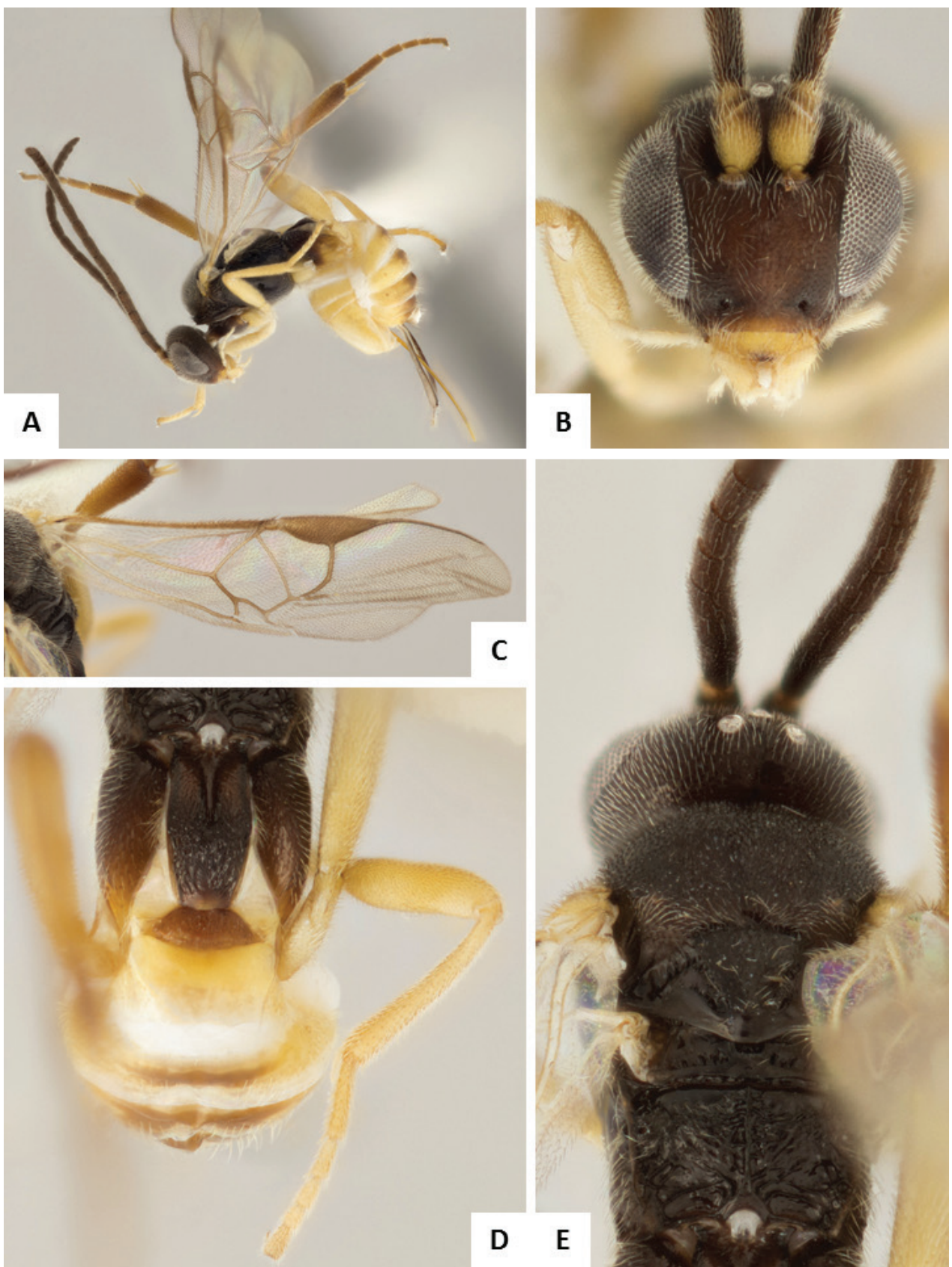

Figure 38. Pseudapanteles gouleti. A Habitus, lateral B Head, frontal C Wings D Metasoma, dorsal E Head and mesosoma, dorsal. 
Material examined. Ontario, Innisville, 45.054942 -76.250619, 6.viii.1963, W. R. M. Mason, Voucher Code: CNCHYM07228; Ottawa, city garden, 45.356 -75.707, 1.ix.2007, H. Goulet, Voucher Code: CAM0254, CAM0258; 10.viii.2007, H. Goulet, Voucher Code: CAM0255, CAM0256, CAM0257; 23-30.vii.2007, H. Goulet, Voucher Code: CAM0883; 23.vii.2007, H. Goulet, Voucher Code: CAM0251, CAM0252, CAM0253; 30.vii-10.viii.2007, H. Goulet, Voucher Code: CAM0874, CAM0875; 45.3561 -75.7069, 23-30.vii.2007, H. Goulet, Voucher Code: CAM0876; 45.399881 -75.697287, Malaise trap, 13-23.vii.2007, H. Goulet, Voucher Code: CNC23941; Ottawa, 45.356083 -75.706933, malaise trap, 13-23. vii.2007, H. Goulet, , Twp. Nepean, Ont, 45.335400 -75.723784, 10.v-25.viii.1949, Tripp, H.A., Voucher Code: CNC309905; Quebec, Hull, 45.428309 -75.713353, 31.viii.1965, Voucher Code: CNCHYM07230.

\section{Rasivalva rugosa (Muesebeck, 1922)}

Fig. 39

Distribution. NEA.

Material examined. Quebec, Summit King Mt. Old Chelsea, 45.503586 -75.797942, 351 m, 17.viii.1965, Voucher Code: CNCHYM03399.

\section{Rasivalva sp.}

\section{Distribution. NEA.}

Notes. This species corresponds in BOLD to BIN BOLD:AAH2147, with all specimens collected in southern Ontario.

Material examined. Ontario, Ottawa, city garden, 45.356100 -75.706900, 10.viii-1.ix.2007, H. Goulet, Voucher Code: WMIC0214; 30.vii-10.viii.2007, H. Goulet, Voucher Code: CAM1011.

\section{Sathon cinctiformis (Viereck, 1911)}

Fig. 40

\section{Distribution. NEA.}

Material examined. Ontario, Ottawa, Dow's swamp , 45.419164 -75.709650, 9.vii.1946, G.S. Walley, Voucher Code: CNC309918; Ottawa, Dow's swamp, 45.394302 -75.704544, 9.vii.1946, G. S. Walley, Voucher Code: CNCHYM03476, CNCHYM03477; Ottawa, 45.406631 -75.701407, 20.vii.1947, W.R.M. Mason, Voucher Code: CNC483601; 20.viii.1947, W.R.M. Mason, Voucher Code: CNC483612; 21.vi.1947, W.R.M. Mason, Voucher Code: CNC483590, CNC483591, CNC483592, CNC483593, CNC483594, CNC483595; 28.vi.1940, O. Peck, Voucher Code: 

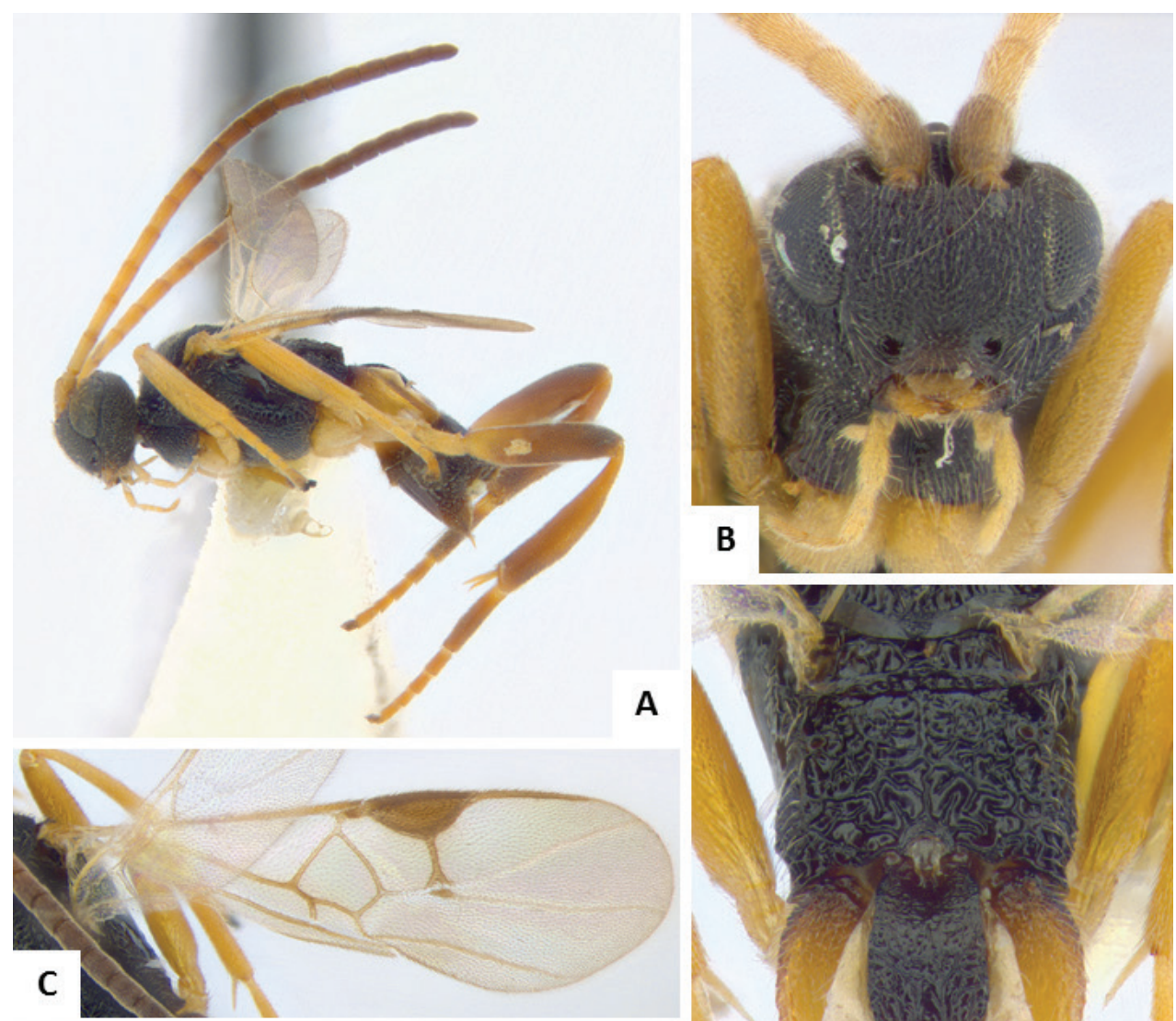

A
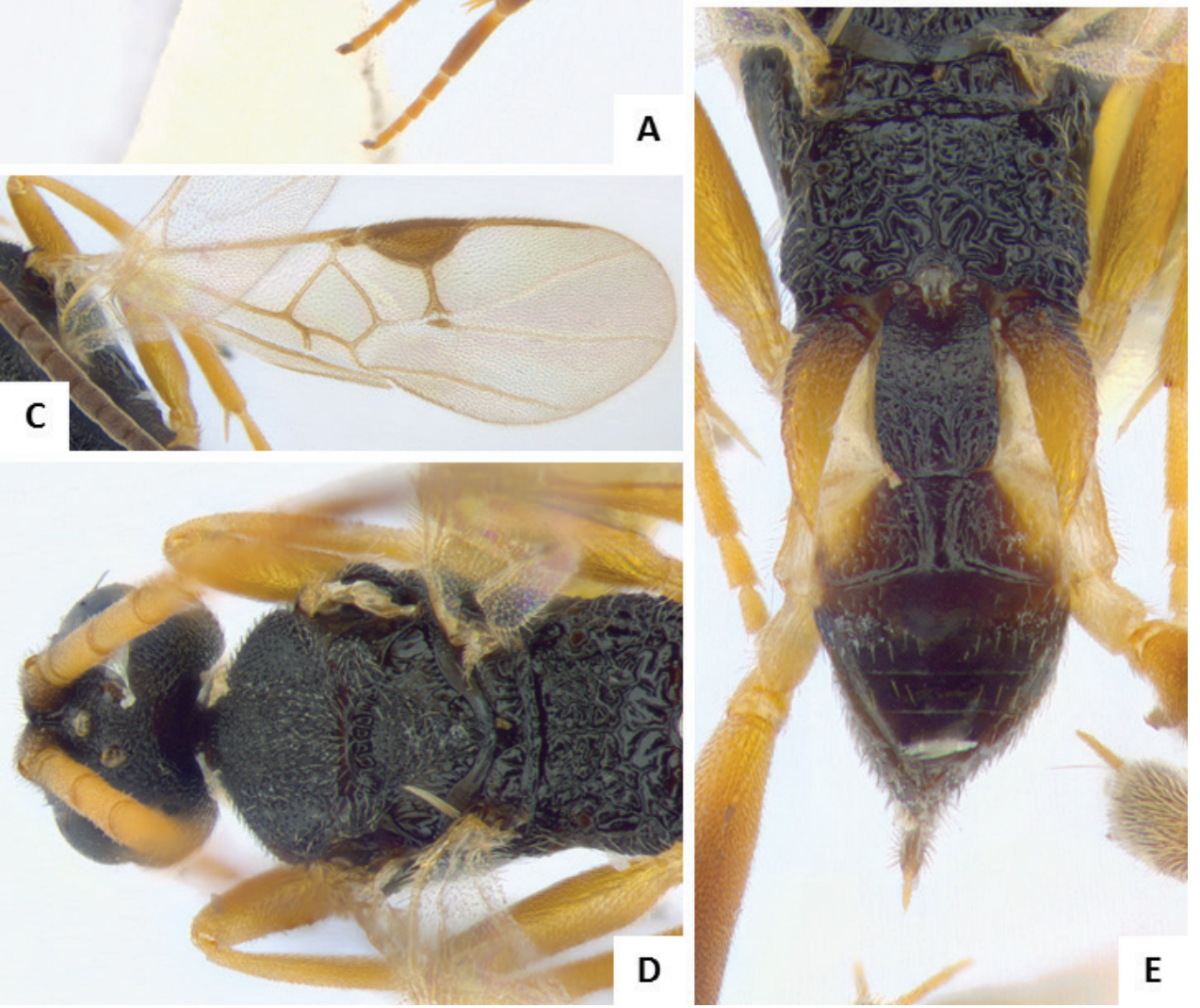

Figure 39. Rasivalva rugosa. A Habitus, lateral B Head, frontal C Wings D Metasoma, dorsal E Head and mesosoma, dorsal. 


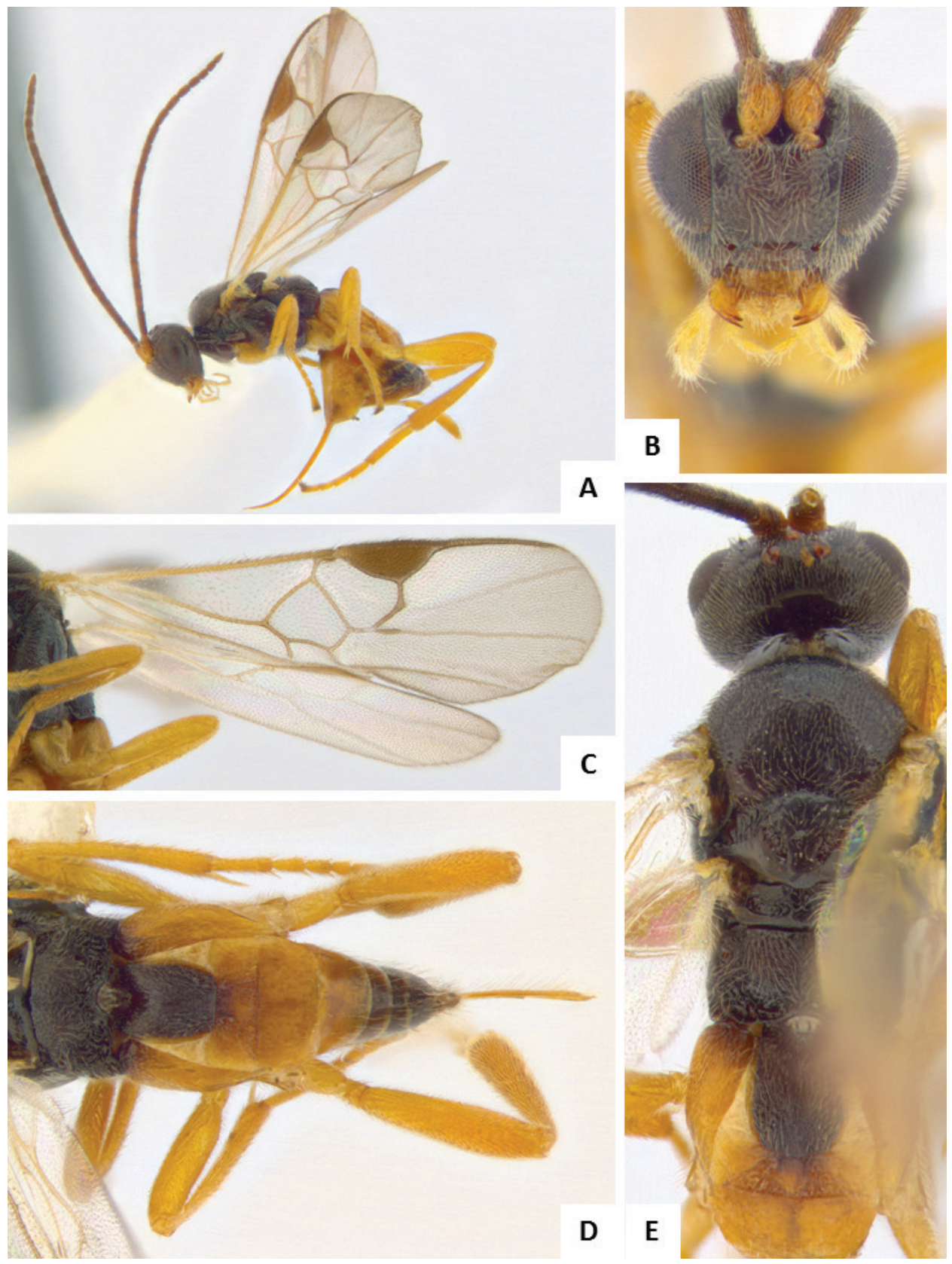

Figure 40. Sathon cinctiformis. A Habitus, lateral B Head, frontal C Wings D Metasoma, dorsal E Head and mesosoma, dorsal.

CNC483599; 5.vii.1947, W.R.M. Mason, Voucher Code: CNC483596, CNC483597, CNC483598; 45.406633 -75.701408, 19.vii.1939, O. Peck, Voucher Code: CNC483600; Quebec, Hull, 45.428550 -75.714554, 11.vi.1957, C.D. Miller, Voucher Code: 
CNC483602, CNC483603, CNC483604, CNC483605, CNC483606, CNC483607, CNC483608, CNC483609; 18.vi.1957, J.G. Chillcott, Voucher Code: CNC483610; 2.viii.1947, W.R.M. Mason, Voucher Code: CNC483611.

\section{Sathon neomexicanus (Muesebeck, 1921)}

\section{Distribution. NEA.}

Material examined. Ontario, Bells Corners, 45.322133 -75.833303, 7.vii.1943, G.S. Walley, Voucher Code: CNC309924.

\section{Acknowledgements}

Many collectors over the past 120 years have contributed Ottawa specimens of Microgastrinae to the $\mathrm{CNC}$, without their efforts this paper would not be possible. We especially thank Henri Goulet and Lubomir Masner (CNC) for collecting most of the recent material (2007-2010). The reviews of John Huber and Donald Lafontaine (CNC) greatly improved the final version of this manuscript. Donald Lafontaine kindly provided the senior author with a list of all Lepidoptera collected in Ottawa as well as comments on its diversity in the region. Ana Fernandez Galliano helped checking the accuracy of labels for some older records. The Biodiversity Institute of Ontario, University of Guelph, has been instrumental in the DNA barcoding of thousands of microgastrine specimens, which has been of great help in taxonomic studies of the Ottawa fauna and many other areas. Two reviewers (Juliano Nunez and James Whitfield) and the editor (Kees van Achterberg) are gratefully acknowledged for helping to improve the final version of the manuscript. This research was supported by project AAFCSTB-1558 'Arthropod systematics research in support of Canadian Agriculture'.

\section{References}

Belcher J, Keddy PA, Catling PFC (1992) Alvar vegetation in Canada: a multivariate description at two scales. Canadian Journal of Botany 70: 1279-1291. doi: 10.1139/b92-161

Fernández-Triana J (2010) Eight new species and an annotated checklist of Microgastrinae (Hymenoptera: Braconidae) from Canada and Alaska. Zookeys 63: 1-53. doi: 10.3897/ zookeys.63.565

Fernandez-Triana J (2014) Towards the conservation of parasitoid wasp species in Canada: Preliminary assessment of Microgastrinae (Hymenoptera: Braconidae). Biodiversity Data Journal 2: e1067. doi: 10.3897/BDJ.2.e1067

Fernandez-Triana JL (2015) A revision of the genus Protomicroplitis Ashmead (Hymenoptera, Braconidae, Microgastrinae), with the description of a new species. Zootaxa 4039(4): 522-542. doi: 10.11646/zootaxa.4039.4.3 
Fernández-Triana J, Goulet H, Bostanian N, Boudreault C (2009) Diversity of Microgastrinae (Hymenoptera: Braconidae) in apple orchards of southern Quebec, Canada. Biocontrol Science and Technology 19: 237-248. doi: 10.1080/09583150802684406

Fernandez-Triana J, Smith MA, Boudreault C, Goulet H, Hebert PDN, Smith AC, et al. (2011) A Poorly Known High-Latitude Parasitoid Wasp Community: Unexpected Diversity and Dramatic Changes through Time. PLoS ONE 6(8): e23719. doi: 10.1371/ journal.pone.0023719

Fernandez-Triana J, Cardinal S, Whitfield J, Smith M, Janzen D (2013) A review of the New World species of the parasitoid wasp Iconella (Hymenoptera, Braconidae, Microgastrinae). ZooKeys 321: 65-87. doi: 10.3897/zookeys.321.5160

Fernandez-Triana J, Shaw M, Cardinal S, Mason P (2014a) Contributions to the study of the Holarctic fauna of Microgastrinae (Hymenoptera, Braconidae). I. Introduction and first results of transatlantic comparisons. Journal of Hymenoptera Research 37: 61-76. doi: 10.3897/jhr.37.7186

Fernandez-Triana J, Penev L, Ratnasingham S, Smith M, Sones J, Telfer A, deWaard J, Hebert P (2014b) Streamlining the use of BOLD specimen data to record species distributions: a case study with ten Nearctic species of Microgastrinae (Hymenoptera: Braconidae). Biodiversity Data Journal 2: e4153. doi: 10.3897/BDJ.2.e4153

Fernandez-Triana J, Janzen D, Hallwachs W, Whitfield J, Smith M, Kula R (2014c) Revision of the genus Pseudapanteles (Hymenoptera, Braconidae, Microgastrinae), with emphasis on the species in Area de Conservación Guanacaste, northwestern Costa Rica. ZooKeys 446: 1-82. doi: 10.3897/zookeys.446.8195

Fernández-Triana J, Whitfield JB, Rodriguez JJ, Smith MA, Janzen DH, Hallwachs W, Hajibabaei M, Burns JM, Solis MA, Brown J, Cardinal S, Goulet H, Hebert PDN (2014d) Review of Apanteles (Hymenoptera: Braconidae, Microgastrinae) from Area de Conservación Guanacaste, northwestern Costa Rica, with keys to all described species from Mesoamerica. ZooKeys 383: 1-565. doi: 10.3897/zookeys.383.6418

Janzen DH, Hallwachs W (2016) DNA barcoding the Lepidoptera inventory of a large complex tropical conserved wildland, Area de Conservacion Guanacaste, northwestern Costa Rica. Genome 59: 641-660. 10.1139/gen-2016-0005

Lafontaine JD (1997) Butterflies and Moths (Lepidoptera). In: Smith IM (Ed.) Assessment of Species Diversity in the Mixedwood Plains Ecozone. Ecological Monitoring and Assessment Network. http://www.naturewatch.ca/mixedwood/lep/intro.htm\#toc [accessed 1.VIII.2010]

Lewis CN, Whitfield JB (1999) Braconid wasp (Hymenoptera: Braconidae) diversity in forest plots under different silvicultural methods. Environmental Entomology 28: 986-997. doi: 10.1093/ee/28.6.986

Mason WRM (1981) The polyphyletic nature of Apanteles Foerster (Hymenoptera: Braconidae): A phylogeny and reclassification of Microgastrinae. Memoirs of the Entomological Society of Canada, Ottawa, Canada, $147 \mathrm{pp}$.

Papp J (1976) A survey of the European species of Apanteles Först. (Hymenoptera, Braconidae: Microgasterinae), I. The species groups. Annales Historico-Naturales Musei Nationalis Hungarici 68: 251-274. 
Ratnasingham S, Hebert PDN (2007) BOLD: The Barcode of Life Data System (www.barcodinglife.org). Molecular Ecology Notes 7: 355-364. doi: 10.1111/j.1471-8286.2007.01678.x Rodriguez, JJ, Fernández-Triana J, Smith MA, Janzen DH, Hallwachs W, Erwin TL, Whitfield JB (2013) Extrapolations from field studies and known faunas converge on dramatically increased estimates of global microgastrine parasitoid wasp species richness (Hymenoptera: Braconidae). Insect Conservation and Diversity 6: 530-536. doi: 10.1111/icad.12003

Shaw SR (2002) Studies of parasitic wasps (Braconidae) in Grand Teton National Park. University of Wyoming National Park Service Research Center Annual Report 26(1): 47-59.

Shaw MR, Stefanescu C, Van Nouhuys S (2009) Parasitoids of European butterflies. In Settele J, Shreeve T, Konvicka M, Van Dyck H (Eds) Ecology of Butterflies in Europe. Cambridge University Press, 130-156.

Smith V, Rycroft S, Brake I, Scott B, Baker E, Livermore L, Blagoderov V, Roberts D (2011) Scratchpads 2.0: a Virtual Research Environment supporting scholarly collaboration, communication and data publication in biodiversity science. ZooKeys 150: 53-70. doi: $10.3897 /$ zookeys. 150.2193

Whitfield JB (1995) Checklist of the Microgastrinae (Hymenoptera: Braconidae) in America north of Mexico. Journal of the Kansas Entomological Society 68: 245-262.

Whitfield JB (1997) Subfamily Microgastrinae. In: Wharton RA, Marsh PM, Sharkey MJ (Eds) Manual of the New World genera of the family Braconidae (Hymenoptera). Special Publication No. 1, International Society of Hymenopterists, Washington, D.C., 333-364. Whitfield JB, Lewis CN (2001) Analytical survey of the braconid wasp fauna (Hymenoptera: Braconidae) on six midwestern US tallgrass prairies. Annals of the Entomological society of America 94(2): 230-238. doi: 10.1603/0013-8746(2001)094[0230:ASOTBW]2.0.CO;2

Whitfield JB (2006) Revision of the Nearctic species of the genus Pholetesor Mason (Hymenoptera: Braconidae). Zootaxa 1144: 1-94.

Yu DSK, van Achterberg C, Horstmann K (2012) Taxapad 2012, Ichneumonoidea 2011. Database on flash-drive. Ottawa, Ontario, Canada. www.taxapad.com

Yu DSK, van Achterberg C, Horstmann K (2016) Taxapad 2016, Ichneumonoidea 2015. Database on flash-drive. Nepean, Ontario, Canada. www.taxapad.com 